\title{
Application of Linear Propagation of Errors to Fuel Rod Temperature and Stored Energy Calculations
}

Prepared by M. E. Cunningham, A. R. Olsen, D. D. Lanning, R. E. Williford

Pacific Northwest Laboratory

Operated by

Battelle Memorial Institute

Prepared for

U.S. Nuclear Regulatory

Commission 


\section{NOTICE}

This report was prepared as an account of work sponsored by an agency of the United States Government. Neither the United States Government nor any agency thereof, or any of their employees, makes any warranty, expressed or implied, or assumes any legal liability or responsibility for any third party's use, or the results of such use, of any information, apparatus product or process disclosed in this report, or represents that its use by such third party would not infringe privately owned rights.

Available from

GPO Sales Program

Division of Technical Information and Document Control

U. S. Nuclear Regulatory Commission

Washington, D. C. 20555

and

National Technical Information Service

Springfield, Virginia 22161 


$$
33679000552804
$$

NUREG/CR-1753

PNL-3539

R3

\section{Application of Linear Propagation of Errors to Fuel Rod Temperature and Stored Energy Calculations}

Manuscript Completed: September 19x

Date Puthistad: October 1900

Prepared by

M. E. Cumingham, A. R. Otsen, D. D. Lanning, R. E. Williford

Pacific Northwest tobotatory

Richland, WA 99352

\section{Prepared for}

Division of Reactor Safety Research

Office of Nuclear Regulatory Research

U.S. Nuclear Regulatory Commission

Washington, D.C. 20555

NRC FIN No. B2043 


\section{ACKNOWLEDGMENTS}

The authors wish to acknowledge the sponsorship of Dr. N. Y. Johnston and H. H. Scott of the Fuel Behavior Research Branch, U.S. Nuclear Regulatory Contistion.

We would like to thank the following individuals from PNL: W. D. Bennett for her help with the computer graphics and $C$. M. Hagen and $\$$. K. Edler for their editing assistance. 


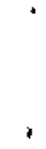

, 


\section{ABSTRACT}

Linear propagation of errors evaluates modeling uncertainty by approxinating a function of interest by first-order Taylor's series expansions and then approximating the variance of the function by the variance of the linear approximation. This report discusses uncertainty analysis for different. nuclear fuel rod designs, the process of model validation, and the effect of cracked pellet fuel models upon temperature uncertainty.

Using a postulated power history, the uncertainty for the predicted thermal response of bolling water reactor (BWR) and pressurized water reactor (PWR) fuel rods was evaluated. Beginning-of-life (BOL) relative uncertainty for BWR and PWR fuel rods is approximately the same, while different end-of-life (EOL) themal response results in different. EOL uncertainty.

Determining the validity of modeling relative to reality is discussed in qualitative terms, Validity is dependent upon verifying that the code correctly implements the model and that satisfactory agreement is found between the model and measurements.

fuel modeling codes are now using cracked pellet fuel models, which result in decreased fuel surface temperature. Estimated stored energy is lowered; but its relative uncertainty is increased. In general, however, the absolute upper uncertainty bound for stored energy is lower for a cracked pellet model than for a solid pellet model. 
. 
SUMARY AND CONCLUSTONS

Linear propagation of errors (LPE) is being used by the Experimental Sup port and Developnent of Single-Rod Fuel Codes Program to evaluate both the uncertainties in fuel rod behavior and in the computer modeling of that behavior. This technique consists of approximating functions of interest by firstorder Taylor's series expansions and then approximating the variance of the function by the yariance of the linear approximation. When applied to an "abstract" (1.e., the basic models) of major fuel rod thermal perfornance codes, this method has been found to be adequate and useful in evaluating uncertainties for model predictions and the influence of various parameters. This report discusses predicted uncertainties for different fuel rod designs, provides qualitative discussion on the process of model validation, and investigates the effect upon tenperature uncertainty when using a cracked pellet fuel model.

A constant neutron flux power history was postulated for studying the predicted response of bolling water reactor (BWR) rod designs $(7 \times 7$ and $8 \times 8)$ and pressurized water reactor (PWR) rod designs $(15 \times 15$ and $17 \times 17)$. Plots of temperature, stored energy, and their associated uncertainties as a function of burnup are presented. Because of the fuel relocation mode1, temperatures decreased early in life with the result that the peak upper uncertainty bound for centerline temperature and stored energy occurred at beginning-of-life $(\mathrm{BOL})$. The PWR rods maintain a constant temperature/power relationship af ter completion of fuel relocation while the Bur rods have an increasing thermal resistance. This difference is due to degradation of the fill gas thernal conductivity. The presence of more helium in prepressurized PuR rods helps to prevent degradation and maintains lower fuel temperatures.

Because fuel temperatures are highly dependent on power, parametric plots of centerline temperature and stored energy uncertainty as a function of linear heat rate uncertainty are presented. At $80 \mathrm{~L}$ all rod designs have approximately the same relative uncertainty. At end-of-life (EOL), the difference in thermal performance between BWR and PWR rods results in differing relative uncertainties. 
Determining the validity of modeling relative to reality is important from a safety standpoint. Three formal definitions are presented here:

- code verification - The process of verifying that a computer code implementation of a model or a set of models is done correctly.

- model analysis - The process of gaining an understanding of the theoretical model, of ten done with sensitivity analysis.

- model validation - The comparison of computer code output to data obtained from an observed physical situation.

A qualitative discussion of model development and validation is based around these three definitions. To illustrate points of the discussion, examples of model validation are included.

Models that better simulate the cracked nature of pellet fuel are cur rently being implemented in fuel themal performance codes. These models change the predicted themal behavior of fuel rods by decreasing the fuel surface temperature (because of fuel relocation closing the gap) and increasing the gap conductance, and by decreasing the effective fuel themal conductivity because of the cracks. These changes affect the uncertainty analysis by reducing the assigned uncertainty on gap conditions and fuel surface temperature, while increasing the assigned uncertainty for fuel themal conductivity. Thus, when comparing solid versus cracked model predictions for the same centerline temperature, the cracked pellet model results in a laver stored energy (because of lower volumetric average fuel temperature) and increased relative uncertainties for predicted centerline temperature and stored energy. Even though the cracked pellet model may have a higher relative uncertainty for stored energy its upper absolute bound is generally lower than that of the solfo pellet model because of the lower predicted value for stored energy. 
CONTENTS

ACKNOWLEDGENTS , . * . . . . . . . ., ij

ABSTRACT * . . + . . . . . . . . . . . *

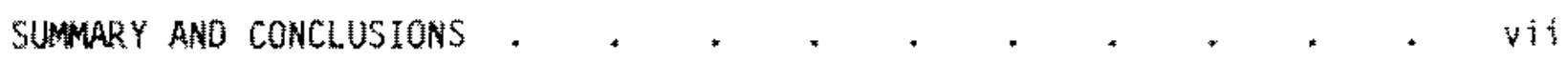

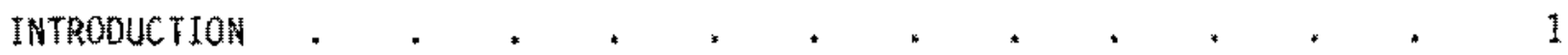

BAKKGROUNO *. . . . . * . . . . . . 3

LINEAR PROPAGATION OF ERRORS * * * * * * * * 3

APPLICATION OF LINEAR PROPAGATION OF ERRORS * . . * * . 6

ASSIGNMENT OF UNCERTAINTY . , . . . . . . . . 8

Local Linear Heat Rate . . . . . . . . 8

Gas Thermal Conductivity . . . . . . . . . 9

Fuel Thermal Conductivity . . . . . . . 9

flux Depression . . . . . . . . . 10

Specific Heat * * . . . . . . * 10

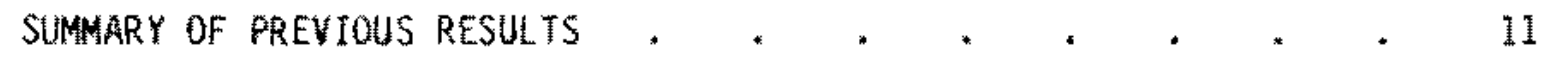

FUEL ROO DESIGN ANALYSIS. . * . . . . . . . . . 12

FUEL ROD TEMPERATURES . . . . . . . . . . . . 20

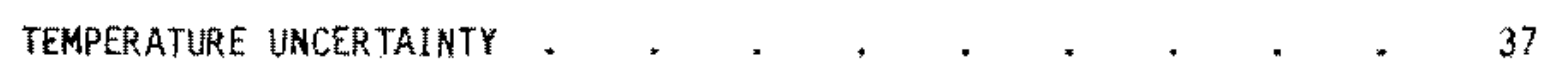

MODEL AND COOE VERIFICATION . . . . . . . . . . . . 45

NODELING ANO REALITF . . . . . . . . * 45

COOE VERIFICATION $*$. . . . * . . * . * 49

MOOEL ANALYSIS. . . . . . . . . . . . . 50

MODEL VALIDATION * * * . * * * , * . 51

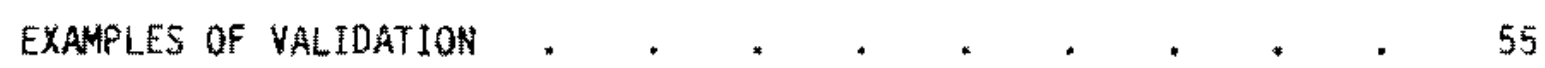

CRACKEU PELLETS ANO UNCERTAINTY ANALYSIS + . . . . . . . . . 65 


REFERENCES
APPENDIX A: THERMAL MODELS IN STORE




\section{FIGURES}

1 Interrelationships of Variables and Mode is in a Fuel

Perfornance Code . . . . . . . . . 7

2 Themal History for BWh $7 \times 7$ (Case 1) . . * . . . 21

3 Thermal History for BWR $8 \times 8$ (Case 1) . . . . . . 22

4 Thermai History for BWR BX8,3 atm (Case 1) . . . . . 23

5 Thermal History for PWR $15 \times 15$ (Case 1) . . . . . . 24

6 Thermal History for PWR $17 \times 17$ (Case 1) . . . . . . 25

7 Thermal History for BWR $7 \times$ (Case 2) . . . . * . 26

8 Themal History for BWR $8 \times 8$ (Case 2) . . . . . * 27

9 Thermal History for BuR $8 \times 8,3$ atm (Case 2) . . . . . 28

10 Themal History for PWR $15 \times 15$ (Case 2) . . . . . . 29

11 Thermal History for PWR $17 \times 17$ (Case 2) . . . . . , 30

12 Thermal History for PWR 17x17, d atm (Case 2) . . . . 31

13 Thernal Resistance History for Case 1 . . . . . . 36

14 Thermal Resistance History for Case 2 . . . . . . 36

15 Beginning-of-Life Relative Uncertainty for an LWR Rod at $20 \mathrm{kw} / \mathrm{m}$. 39

16 Beginning-of-Life Relative Uncertainty for an LWR Rod at $35 \mathrm{kw} / \mathrm{m}$. 39

17 Beginning of-Life Relative Uncertainty for an LWR Rod at $50 \mathrm{~kW} / \mathrm{m}$. 40

19 End-of-Life Relative Uncertainty for a BhR Rod at $20 \mathrm{~kW} / \mathrm{m}$. . 42

19 End-of-Life Relative Uncertainty for BhR Rod at $35 \mathrm{kw} / \mathrm{m}, \quad . \quad 42$

20 End-of-Life Relative Uncertainty for a BWR Rod at $50 \mathrm{~kW} / \mathrm{m}$. , 43

21 End-of-Life Relative Uncertainty for a PWR Rod at $20 \mathrm{~kW} / \mathrm{ml}$. . 43

22 End-of-Life Relative Uncertainty for PWR Rod at $35 \mathrm{~kW} / \mathrm{m}$. . 44

23 End-of-Life Relative Uncertainty for a PWR Rod at $50 \mathrm{~kW} / \mathrm{m}$. . 44

24 Components of Modeling Process . . . . . . . . 46 
25 Computer Code Inplenentation of Theoretical Model . . * . 50

26 Verification of Steady-State Fuel Radial Temperature Profile Model 56

27 Validation of Gas Thernal Conductivity Model . . . . . 57

28 Example of Valldation of a Fuel Performance Code . . . . . 58

29 Comparison of GAPCON-THERMAL -3 to IFA-513 Startup Data. . . . 62

30 Comparison of FRAPCON-1 to IFA-513 Startup Data * . . . 63

31. Minimun Conductivity Multiplier values as a Function of

32 BWR 8x8 Stored Energy Based on Solld and Cracked Pellet Models . 72

33 BuR 8,8 Stored Energy Upper Absolute Uncertainty Bounds . . . 72

B.1 Components of Resistance. $*$. * . . . * . B.2

Q.2 Resistance Behavior of Various Rods . . . . . . . . . B.2

C.1 Fill Gas Composition History for BHR $7 \times 7$, Case 2. . . . C.2

C.2 Fill oas Composition History for BWR $8 \times 8$, Case $2 *$ * . . C.2

C.3 Fill Gas Compostion history for 3 atm BuR 8xs, Case 2. . . C.3

C.4 Fill Gas Composition History for PWR 15x15, Case 2 . . = C.3

C.5 Fill Gas Composition History for PWR $17 \times 17$, Case 2 * . . C.4

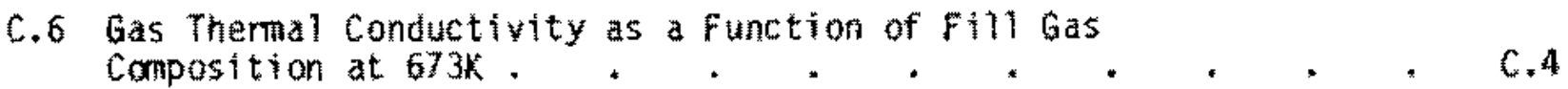




\section{TABLES}

1 Fuel Rod Parameters for Comparison Study . . . . . 12

2 Assumed Power Histories for Rod Type Comparison, Warying

Peak Powers * . * . . . * . . . . 14

3 Assuned Power Histories for Rod Type Comparison, $35 \mathrm{kWhm}$ Peak Power . . . * . . . . . * * 15

4 Assumed Input Uncertainties Common to All Rod Types * . . 16

5 Gas Thermal Conductivity uncertainty, Varying peak power . . 17

6 Gas Thermal Conductivity uncertainty. $35 \mathrm{kw} / \mathrm{n}$ Peak Power . . 18

7 Uncertainty for water Film and Codding Temperatures at $35 \mathrm{~kW} / \mathrm{n} .+.+.+.+., 19$

8 Elopsed Time and Burnup, Varying Pak Power . . . . . . . 32

9 Elapsed Time and Burnup, $35 \mathrm{kw} / \mathrm{m} \mathrm{Peak}$ Power * . $*$. . 33

10 GT3 Predicted Relocation as a Function of Burnup . . . . . 34

11 Sasic Assumptions for BoL uncertainty Parametric Study . * . 41

12 Basic Assumptions for EoL Uncertainty Parametric Study . . . . 41

Assumptions of Parametric Plots of Minimum $C_{F}$ Versus
Resistance

14 Effect of Cracked Pellet Model on Relative uncertalnties * * 70 


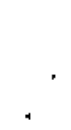


INTRODUCTION

During off-nomal conditions in a nuclear reactor, the integrity of the fuel rods is highly dependent upon how high cladding temperatures becone. These peak temperatures are dependent upon fuel rod conditions at the beginning of the off-normal period. To assess these initial conditions, vendors and regulators incorporate mathenatical models into large computer codes; and to help assure appropriate use of these codes, the user must understand their accuracies and limitations.

In 1974, the Fuel Behavior Research Branch of the Nuclear Regulatory Comission (NRC) began a program to verify the accuracy of fuel performance computer codes and identify their limitations. This program, Experinental Support and Development of single-Rod Fuel Codes, (a) is being conducted at Pacific Northwest Laboratory (PNL). (b) The progran began with the irradiation of two fuel assenthes to extend the base of infomation regarding the behavior of reactor fuel during steady-state operation. This data base is being used to verify steady-state fuel perfomance compidter codes (Hann et al. 1977). Since 1974 two more instrumented fuel assemblies have been included in the program. Steady-state and transient temperature data from these assenbiles have been obtained and analyzed, ex-reactor testing to determine mechanical behavior of the fuel rod has begun, and error and uncertainty analysis has received increasing emphasis.

In 1977, program staff began estimating the uncertainty of thermal perfomance predictions that are based on steady-state fuel perfornance computer codes. This analysis utilized a technique called linear propagation of errors (LPE), which was found to be useful in predicting uncertainty. The work culfininated with a report (Cunningham et al, $1978^{(c)}$ ) that discussed how LPE could be applied to steady-state fuel codes and provided some results for typical boiling water reactor (BWR) fuel rod.

(a) Prior to Fy1980, this program was Experimental Verification of Steady-State codes.

(b) Operated for the U.\$. Department of Energy (DOE) by Battelle Menorial Institute.

(c) Hereafter, this report will be referred to as PNL -2581 . 
The work reported in PNL -2581 is expanded in this report in three nain areas. First, this report compares the responses of BWR and pressurized water reactor (PWR) fuel rods during wo postulated power histories. Second, a qualitative statement is presented discussing the processes of code verification and validation. Different approaches to answering the question of how the code relates to reality are presented. Finally, advancements in data analysis and fuel modeling over the last few years have shown that fuel cracking and relocation affect the themal behavior of a fuel rod; therefore, this report includes a discussion on the effect of this concept change upon uncertainty analysis. 


\section{BACKGROUNO}

The work presented in this report relies heavily on the methodology developed and reported by Cunningham (PNL-2581). This section provides a summ rary of the LPE method, the ways it is applied, and sample results.

\section{LINEAR PRDPAGATION OF ERRORS}

A comon method used to predict the radial temperature profile of a fuel rod includes beginning with the coolant temperature and then, working radially inward, calculating the temperatures of different fuel rod regions. (a) Thus: the calculations for a particular region, such as the gap between fuel and cladding, (b) are dependent upon the calculations that preceded. This calculational scheme readily lends itself to estimating uncertainty by LPE. With this technique, estimated uncertainties are dependent upon input parameters and uncertainties and calculated yariables and uncertainties. In addition, it is relatively easy to determine what percentage of an uncertainty estiriate is due to particular variabie of interest.

LPE consists of first approxinating the function of interest by a first. order Taylor's series and then approximating the variance of the function by the variance of the linear approximation. That is, if $y$ is a function of $p$ variabus

$$
Y=F\left(x_{1}, x_{2}, \ldots, x_{p}\right)
$$

then it thay be linearly approximated as

$$
p_{1}=F\left(\xi_{1}, \xi_{2}, \ldots, \xi_{p}\right)+\left(x_{1}-\xi_{1}\right) \frac{\partial F}{\partial X_{1}}+\left(x_{2}-\xi_{2}\right) \frac{\partial F}{\partial x_{2}}+\ldots+\left(x_{p}-\xi_{p}\right) \frac{\partial F}{\partial X_{p}}
$$

(a) See Section ? of PNL-2561

(b) Hereafter referred to as the fuel-cladding gap 
where the $x_{i}$ s are randon variables that may or nay not be interdependent and where

$$
\frac{\partial F}{\partial x_{i}}=\frac{\partial F\left(x_{1}, x_{2}, \cdots, x_{n}\right)}{\partial x_{i}}
$$

is evaluated at $\left(x_{1}, x_{2}, \ldots, x_{p}\right)=\left(\xi_{1}, \xi_{2}, \ldots, \xi_{p}\right)$ where the $\xi_{1}$ are the mean values or expected values of the $x_{i} s$.

Next, the absolute variance of $y$ is approximated by the absolute varlance of the linear approximation

$$
\sigma_{Y}^{2}=\sigma_{Y_{L}}^{2}=\sum_{i=1}^{p}\left(\frac{\partial F}{\partial x_{j}}\right)^{2} \sigma_{i}^{2}+2 \sum_{\substack{i=1 \\ j>i}}^{p-1}\left(\frac{\partial F}{\partial x_{i}}\right)\left(\frac{\partial F}{\partial x_{j}}\right) \sigma_{i j}
$$

where $\sigma_{i}^{2}=$ the absolute variance of $x_{i}$ $\sigma_{i j}=$ the covariance between $x_{i}$ and $x_{j}$.

The validity of this approximation of the variance of $y$ depends on the following conditions:

- The variabies $x_{i}$ have finjte means $\left(\mu_{i}\right)$ and variances $\left(o_{i}{ }^{2}\right)$.

- The standazd deviations of the $x_{j}\left(o_{i}=\sqrt{\sigma_{i}^{2}}\right)$ are smail relative to their means (u).

The latter is concerned with the adequacy of the approximation; that is, with how closely the sumed It near terms of the Taylor's series expansion $\left(Y_{1}\right)$ approximate the exact function $Y$ aver the range of variables in question. In terms of uncertaifties, as defined in this report, the second requirement is that the $x_{i}$ uncertainties be small.

The relative variance of $\left(C_{y}{ }^{2}\right)$ may now be defined as 


$$
c_{y}^{2}=\frac{\sigma_{y}^{2}}{u_{y^{2}}^{2}}
$$

For internediate variables that are interrelated, the relative covariance (cif) is found by

$$
C_{i j}=\frac{\sigma_{i j}}{H_{i j j}}
$$

in this report, the percent uncertainty for a variable is determined fron its re? ative variance by

$$
u_{Y}=300 \sqrt{c_{Y}^{2}}
$$

This uncertainty estimate has a conflatence level of $99.7 \%$. In other words, assuming $Y$ is nomally distributed, $Y$ will fall within the bounds of Hy $+3 \%$ y $99.7 \%$ of the time. In this reports it is called "zaconfidence level."

Four conditions are Important in obtaining meanimgful results from the described nethod of linear propagation of uncertainty.

- For each input variable, the error (uncertainty) distribution is assuned Gassian about a mean value. Moreover, the uncertainty is described atequately by the relative variance $\mathrm{c}_{i}^{2}=\sigma_{i}{ }^{2} / \mathrm{H}_{i}$. This implies that for the percent uncertainty to renath constant, the absolute variance must increase as the nean value increases.

- There is no covariance between any of the input variables; that is, there are no correlations between measurenent eryors for input. varatioles.

- For the functions of interest the propagation of errors by firstorder Taylor's series provides an adequate estimate of uncertaluty. 
- The uncertainty distributions for the derived variables (y) are suf ficiently symetric for the first condition to be true.

The last of these conditions is related to determining the uncertainties for calculated values of themal perfomance. If identical experiments could be run and parameters of interest could be neasured under exac condtions, (burnup, time, power, etc.) for each experiment, a distribution of values for the vartable could be obtained this may be simulated by Monte Carlo techniques). Under this condition, such a distribution would be approximately symetric about the nean value for the conditions of the experiment.

It is important to undersiand that LPE alone does not provide an estinate of bias between model and data; however, it does provide an estimate of the width of a nomal distribut on about the mean value of a calculated variable. To estimate bas, researchers iust compare this predicted or modeled distribution with an observed distributhon. (See Model and Code Verification section.)

APPLICATION OF LINEAR PROFAGATION OF ERRORS

This report includes a general uncertainty analys tures and stored energy. A typlcal steady-state fuel performance code involves a complexity of rodels and iterative solutions that make application of LPE nearly impossible (see figure 1). Therefore, the analysis was applied only to basic models. Since basic nodels are generally applicable to all fuel performance codes, LPE has in effect been applied to an abstract of fuel perfomance modeling. (Selected nodels are discussed in Appendix A.)

After the models of interest are chosen, the uncertainty equations are derived; these complex equations can be found in Appendix $B$ of PN-2581 and, therefore, are not repeated here. To facilitate the uncertainty analysis the models and uncertainty equations have been combined into the computer code STORE.

It should be recognized that alchough all codes may work from the same bast models, each code may use different material properties, have different. secondary models, and apply different techniques for solution. Therefore, 


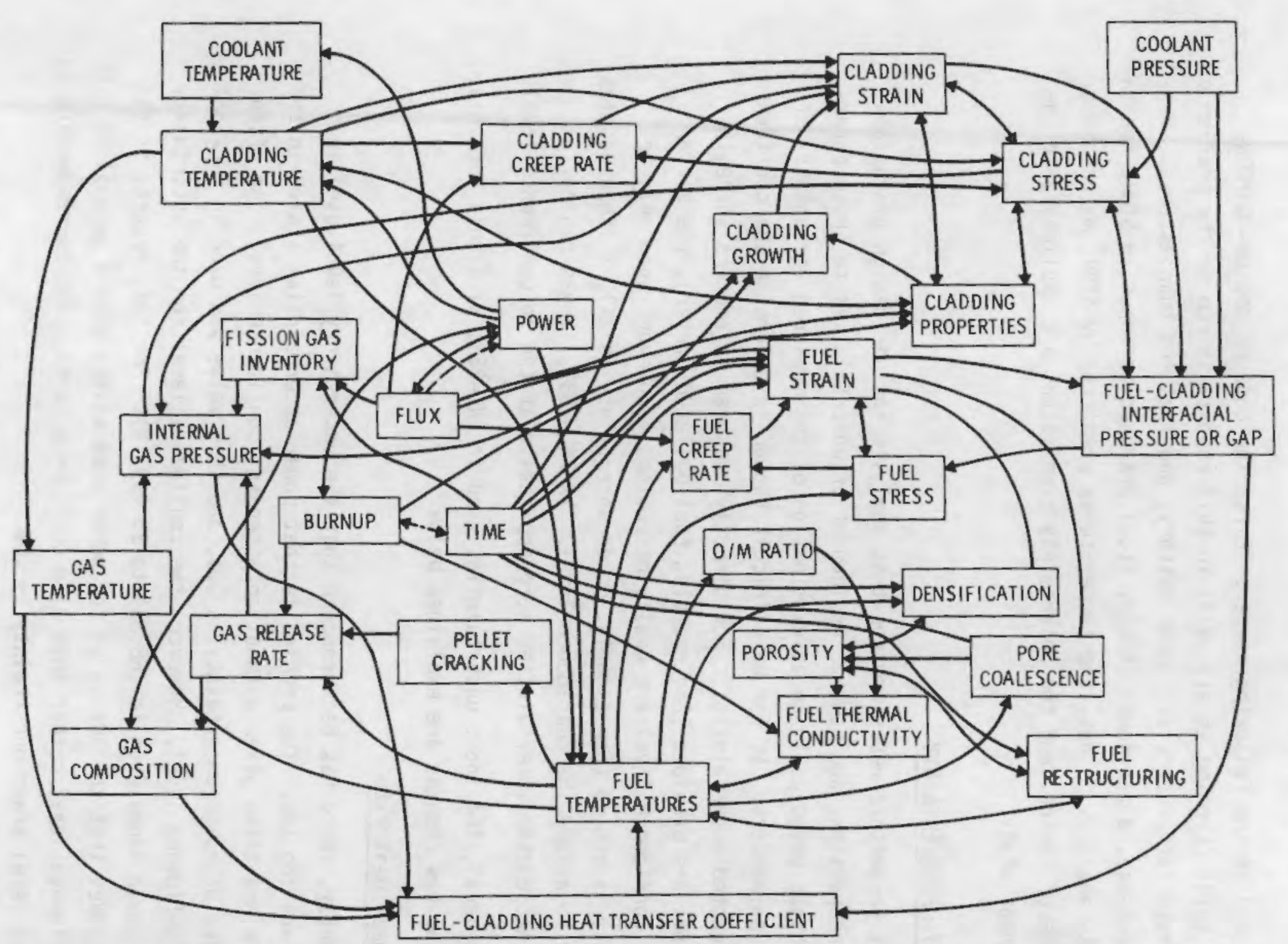

FIGURE 1. Interrelationships of Variables and Models in a Fuel Performance Code 
STORE is used in the following manner: First the code of choice--GAPCONTHERMAL-3 (GT3) (Lanning et al. 1978) in this report--is run on the problem of choice. Material properties, power history, and fuel rod changes (e.g., fission gas release, dimensional change, etc.) are used as input to STORE for the uncertainty analysis. Thus, the temperatures predicted by STORE match those of the code of choice, and the uncertainty predictions are applicable to the problem under study.

\section{ASSIGNMENT OF UNCERTAINTY}

Input parameters necessary for this analysis include design dimensions, material properties, and power. Assignment of uncertainties to these parameters is based principally on an examination of the measured variability of the input parameters. It can be difficult though to determine the confidence level of quoted uncertainties. For directly measured parameters where the measurements are provided (for example, fuel pellet diameter), the variance $\left(\sigma_{x}{ }^{2}\right)$ and confidence level are easily determined. However, when only an uncertainty is quoted (for example, power uncertainty is $Z \%$ ), it then becomes necessary to assume the confidence level. In PNL-2581, it was generally assumed that quoted uncertainties were applicable at the $3 \sigma$ confidence level.

In general, the input uncertainties used in PNL-2581 will hold for this report; the few changes are explained below.

\section{Local Linear Heat Rate}

Recently, there has been concern that the uncertainty level used in PNL-2581 was too low. The problem is that power uncertainties stated in the literature are often given without an attendant confidence level. An of ten found value of power uncertainty is $10 \%$, and this value was used in PNL-2581 at a $3 \sigma$ confidence level. However, the confidence level for the uncertainty estimate could range from $10(68.3 \%)$ to $30(99.7 \%)$. For this report, it has been concluded that the $10 \%$ value for power uncertainty should be applied with a $2 \sigma$ confidence level rather than a $3 \sigma$ confidence level. The corresponding $3 \sigma$ confidence level power uncertainty is $15 \%$. 


\section{Gas Thermal Conductivity}

In PNL-2581, the uncertainty for gas thermal conductivity was based on the combined uncertainty of the gas mixture present in the fuel-cladding gap and the uncertainty in the prediction of the thermal conductivity for the assumed gas mixture. It was assumed that gas mixture uncertainty varied linearly from $0 \%$ at beginning-of-life (BOL) to $25 \%$ at end-of-life (EOL) while the model uncertainty varied from 2 to $6.5 \%$ for gases ranging from pure helium to typical fission gas mixtures; all of which resulted in a steadily increasing gas themal conductivity uncertainty with burnup.

However, it may reasonably be assumed that at a certain point the gas composition will be nearly saturated with fission gas and the thermal conductivity uncertainty may be reduced. Therefore, a new method has been derived to assign gas thermal conductivity.

The primary question still remains: What is the gas composition uncertainty? The method now used is based on the following: First, the Beyer-Hann fission gas release model (Beyer and Hann 1974) is used in GT3 for temperatures greater than 1473K; for lower temperatures, the gas release model of Bellamy and Rich (1969) is used. Next, it was observed that for the GT3 runs being evaluated, predicted temperatures exceeded 1473K only at low burnup; therefore, the low-temperature gas release model was operative during the bulk of the GT3 modeling. Beyer and Hann assign an uncertainty of $1 \%$ to the low-temperature gas release mode1. This uncertainty is applied to the GT3 estimate of fission gas moles released, which produces upper and lower estimates of the amount of fission gas in the gap. Finally, the limits on gas composition are converted to themal conductivities, and an uncertainty estimate is obtained by comparing the original thermal conductivity to the calculated limits. A rinimum uncertainty of $2 \%$ is maintained. Appendix $C$ provides further discussion and plots of fill gas composition as a function of burnup.

\section{Fuel Themal Conductivity}

At $\mathrm{BOL}$, the uncertainty for fuel thermal conductivity based on examination of data from unirradiated $\mathrm{UO}_{2}$ is $10 \%$ (Hagrman and Reymann 1979). During 
irradiation, the effective thermal conductivity of the fuel matrix is expected to change because of fuel cracking and relocation. In addition, the entrapment of fission products within the $\mathrm{UO}_{2}$ lattice may change the thermal conductivity of individual chunks of $\mathrm{UO}_{2}$. However, examination of burnup effects on the thermal conductivity of solid pieces of $\mathrm{UO}_{2}$ reveais little effect above 1100K (Lokken and Courtright 1977). It is therefore concluded that the major effects on $\mathrm{UO}_{2}$ thermal conductivity are caused by cracking and relocation. From anaiysis of in-reactor thermal data, it has been concluded that cracking and relocation are essentially concluded by $430 \mathrm{GJ} / \mathrm{kgU}$ (5000 MNd/MTM) of burnup. In addition, reductions of up to $20 \%$ have been deduced for the effective $\mathrm{UO}_{2}$ thermal conductivity (Williford et al. 1980). Therefore, the uncertainty applied in this report for $\mathrm{UO}_{2}$ thermal conductivity will vary linearly with burnup from $10 \%$ at $\mathrm{BOL}$ to $20 \%$ at $430 \mathrm{GJ} / \mathrm{kgU}$; and $20 \%$ uncertainty will be assumed for all burnups greater than $430 \mathrm{GJ} / \mathrm{kgU}$.

\section{Flux Depression}

During irradiation the flux depression within the fuel pellet primarily changes due to neutronics effects such as U-235 depletion and Pu build-in. Secondary causes of flux depression change might be physical effects such as fuel densification and relocation. Physical changes occur during the first $430 \mathrm{GJ} / \mathrm{kgJ}$ of burnup while the neutronics changes occur over the lifetime of the fuel.

The flux depression uncertainty for this report will increase linearly from $5 \%$ at $\mathrm{BOL}$ (based on neutronics considerations) to $10 \%$ at $430 \mathrm{GJ} / \mathrm{kgU}$. This may provide an initially high uncertainty number but should account for any effects throughout the postulated irradiation.

Specific Heat

Under steady-state conditions, specific heat affects the stored energy. From evaluation of data, the specific heat of $\mathrm{UO}_{2}$ has an uncertainty of $5 \%$ at BOL (Kerrisk and Clifton 1972); for this report, it will increase linearly from $5 \%$ at BOL to $10 \%$ at $432 \mathrm{GJ} / \mathrm{kgU}$ to account for any factors that could change the specific heat. 


\section{SUMMARY OF PREVIOUS RESULTS}

The work presented in PNL-2581 discussed the uncertainties in the predicted response of a BWR- 6 fuel rod during startup and steady-state irradiation. The principal objectives of that work were to apply LPE to a steadystate fuel code, evaluate the applicability of LPE, and determine which code variables are most important to the uncertainties of predicted variables.

It was concluded that when compared to Monte Carlo evaluations, LPE is an adequate means to estimate uncertainty. Because LPE is applied to basic thermal models, the uncertainty predictions are generally applicable to steadystate fuel codes rather than being specific to only one code. Other conclusions that were reached include

- Good agreement was observed between the LPE uncertainty estimates for gap conductance and the observed variability of gap conductance as inferred from experimental data.

- Linear power, fuel thermal conductivity, and neutron flux depression (radial power profile) are the most influential parameters upon fuel centerline temperatures.

- The maximum uncertainties that occur are time dependent and generally increase with time. 


\section{FUEL ROD DESIGN ANALYSIS}

The light water reactor (LWR) industry uses several basic fuel rod designs and operating conditions, which are modified as necessary from reactor to reactor. Designs can differ in dimension, fuel type, and power history. Because of these differences, questions arise as to their effects on thermal performance and uncertainty. This section presents an analysis of some standard designs and thereby a cursory and general evaluation of LWR fuel rod designs.

The selection of standard designs for an analysis of this type can be troublesome; however, four designs were specified during the assessment of

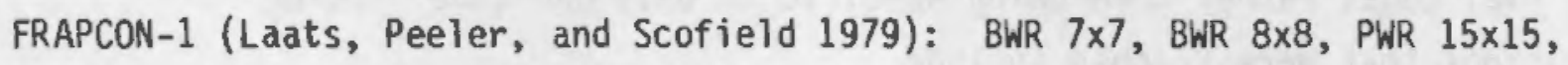
and PWR 17x17. These and two additional designs were analyzed. BWR fuel rods are normally backfilled with helium at 1-atm pressure. Future BWR rods, however, may be backfilled at $3 \mathrm{~atm}$ to reduce the thermal effects of fission gas release; therefore, a BWR $8 \times 8$ rod with an initial pressure of 3 atm was included. PWR rods are normally backfilled with helium at 15-25 atm to counteract the high external pressure; for contrast, a PWR $17 \times 17$ rod at 1-atm pressure was included. Table 1 lists the fuel rod parameters used in this study.

IABLE 1. Fuel Rod Parameters for Comparison Study

\begin{tabular}{|c|c|c|c|c|}
\hline Parameter & BWR $7 \times 7$ & BWR $8 \times 8$ & PWR $15 \times 15$ & PWR $17 \times 17$ \\
\hline Cladding outer diameter, $\mathrm{mm}$ & 14.30 & 12.52 & 10.77 & 9.50 \\
\hline Cladding inner diameter, $\mathrm{mm}$ & 12.67 & 10.80 & 9.50 & 8.36 \\
\hline Fuel diameter, mm & 12.37 & 10.57 & 9.31 & 8.19 \\
\hline Fuel enrichment, $\%$ & 2.2 & 2.2 & 2.6 & 2.6 \\
\hline Fuel density, OTD $_{2}$ & 94 & 95 & 94 & 95 \\
\hline Plenum volume, $\mathrm{cm}^{3}$ & 37.5 & 21.0 & 9.2 & 6.6 \\
\hline Fill gas pressure, $\mathrm{MPa}$ & 0.10 & $0.10,0.30$ & 2.38 & $0.10,2.03$ \\
\hline Coolant pressure, $\mathrm{MPa}$ & 7.14 & 7.27 & 15.51 & 15.51 \\
\hline Coolant temperature, $\mathrm{K}$ & 551 & 551 & 561 & 561 \\
\hline Fuel/length, $\mathrm{kg} / \mathrm{m}$ & 1.239 & 0.914 & 0.702 & 0.549 \\
\hline
\end{tabular}


This type of comparative examination must include power history. In an operating reactor, an LWR fuel rod will be exposed to a number of power cycles, several different sustained power levels, and shifts in core position. Thus, fuel rods from different reactors may have significantly different power histories. It would be impractical to try to duplicate this variety of power histories; however, since the general objective is to operate power reactors at constant power, the following assumptions were used to construct a useful power history:

- The rods are exposed to a constant neutron flux throughout the assumed irradiation exposure.

- Because of fuel depletion, each rod will experience decreasing power during the exposure.

- All rods are irradiated to a burnup of $2590 \mathrm{GJ} / \mathrm{kgU}$ (30 GWd/MTM).

- Two different initial rod peak powers are assumed: the core average for the rod design as stated in Table II of the assessment of FRAPCON-1 (Case 1 ) and $35 \mathrm{~kW} / \mathrm{m}$ (Case 2).

Tables 2 and 3 summarize the assumed power histories.

As stated previously, the procedure for the uncertainty analys is is to first run the code of choice and then match STORE to the code results. The resulting uncertainty estimates from STORE may be considered applicable to the fuel performance code if appropriate input uncertainties are provided to STORE. (a) Tables 4-6 list the assumed input uncertainties for the rod type analysis.

Table 7 lists predicted uncertainties for the water film temperature rise $\left(\Delta T_{F}\right)$, the cladding temperature rise $\left(\Delta T_{c}\right)$, and the cladding inner surface temperature $\left(T_{C}\right)$. Several items are noteworthy.

(a) Assumes that the basic models in the code of choice are equivalent to those in STORE. 
TABLE 2. Assumed Power Histories for Rod Type Comparison, Varying Peak Powers

\begin{tabular}{|c|c|c|c|c|c|c|c|}
\hline \multicolumn{2}{|c|}{ BWR 7×7 } & \multicolumn{2}{|c|}{ BWR $8 \times 8$} & \multicolumn{2}{|c|}{ PUR $15 \times 15$} & \multicolumn{2}{|c|}{ PWR $17 \times 17$} \\
\hline$B u^{(a)}$ & PLHR ${ }^{(b)}$ & BU & PLHR & BU & PLHR & BU & PLHR \\
\hline 0 & 0.0 & 0 & 0.0 & 0 & 0.0 & 0 & 0.0 \\
\hline 0 & 6.6 & 0 & 6.6 & 0 & 6.6 & 0 & \\
\hline 0 & 13.1 & 0 & 13.1 & 0 & 13.1 & 0 & 13.1 \\
\hline 0 & 19.7 & 0 & 19.7 & 0 & 19.7 & 0 & 19.7 \\
\hline 1 & 26.3 & 1 & 26.3 & 1 & 26.3 & 1 & 25.0 \\
\hline 2 & 34.0 & $\overline{1}$ & 29.0 & 1 & 32.0 & 4 & 25.0 \\
\hline 13 & 34.0 & 3 & 29.0 & 4 & 32.0 & 22 & 25.0 \\
\hline 26 & 34.0 & 15 & 29.0 & 21 & 32.0 & 44 & 25.0 \\
\hline 53 & 34.0 & 31 & 29.0 & 43 & 32.0 & 89 & 25.0 \\
\hline 107 & 33.9 & 62 & 29.0 & 88 & 32.0 & 177 & 24.8 \\
\hline 187 & 33.7 & 124 & 28.9 & 175 & 31.8 & 266 & 24.7 \\
\hline 266 & 33.6 & 216 & 28.7 & 263 & 31.7 & 353 & 24.6 \\
\hline 346 & 33.4 & 308 & 28.6 & 350 & 31.6 & 441 & 24.5 \\
\hline 425 & 33.3 & 399 & 28.4 & 437 & 31.5 & 528 & 24.4 \\
\hline 503 & 33.1 & 490 & 28.3 & 524 & 31.3 & 615 & 24.3 \\
\hline 581 & 33.0 & 581 & 28.1 & 610 & 31.2 & 702 & 24.3 \\
\hline 659 & 32.8 & 671 & 28.0 & 696 & 31.1 & 788 & 24.2 \\
\hline 737 & 32.7 & 760 & 27.8 & 782 & 31.0 & 874 & 24.1 \\
\hline 814 & 32.5 & 849 & 27.7 & 867 & 30.8 & 959 & 23.9 \\
\hline 891 & 32.4 & 938 & 27.6 & 952 & 30.7 & 1044 & 23.8 \\
\hline 967 & 32.2 & 1026 & 27.4 & 1036 & 30.6 & 1129 & 23.7 \\
\hline 1043 & 32.1 & 1114 & 27.3 & 1121 & 30.5 & 1213 & 23.6 \\
\hline 1119 & 32.0 & 1201 & 27.1 & 1205 & 30.4 & 1297 & 23.5 \\
\hline 1195 & 31.8 & 1288 & 27.0 & 1288 & 30.3 & 1381 & 23.4 \\
\hline 1270 & 31.7 & 1375 & 26.8 & 1372 & 30.2 & 1464 & 23.3 \\
\hline 1345 & 31.5 & 1460 & 26.7 & 1455 & 30.0 & 1547 & 23.2 \\
\hline 1419 & 31.4 & 1546 & 26.6 & 1537 & 29.9 & 1629 & 23.1 \\
\hline 1493 & 31.2 & 1631 & 26.4 & 1620 & 29.8 & 1712 & 23.1 \\
\hline 1567 & 31.1 & 1716 & 26. & 1702 & 29. & 1794 & 23.0 \\
\hline 1639 & 31.0 & 1800 & 26.2 & 1783 & 29.6 & 1875 & 22.9 \\
\hline 1714 & 30.8 & 1883 & & 1865 & 29.4 & 1957 & 22.8 \\
\hline 1786 & 30.7 & 1967 & 25 & 1946 & 29.3 & 2038 & 22.7 \\
\hline 1859 & 30.6 & 2050 & 25. & 2026 & 29.2 & 2118 & 22.6 \\
\hline 1931 & 30.4 & 2132 & & 2107 & 29.1 & 2198 & 22.5 \\
\hline 2003 & 30.3 & 2214 & 2 & 2187 & 29.0 & 2278 & 22.4 \\
\hline 2074 & 30.2 & 2296 & & 2267 & 28. & 2358 & 22.3 \\
\hline 2146 & 30.0 & 2377 & & 2346 & 28.8 & 2437 & 22.2 \\
\hline 2217 & 29.9 & 2459 & 25 & 2425 & 28.6 & 2516 & 22.1 \\
\hline 2283 & 29.8 & 2538 & 25.0 & 2504 & 28.5 & 2595 & 22.0 \\
\hline 2358 & 29.6 & & & 2583 & 28.4 & & \\
\hline
\end{tabular}

(a) BU = burnup in $\mathrm{GJ} / \mathrm{kgU}$

(b) $P L H R=$ rod peak linear heat rate in $\mathrm{kW} / \mathrm{m}$ 
TABLE 3. Assumed Power Histories for Rod Type Comparison, $35 \mathrm{~kW} / \mathrm{m}$ Peak Power

\begin{tabular}{|c|c|c|c|c|c|c|c|}
\hline \multicolumn{2}{|c|}{ BWR 7 $\times 7$} & \multicolumn{2}{|c|}{ BWR $8 \times 8$} & \multicolumn{2}{|c|}{ PWR $15 \times 15$} & \multicolumn{2}{|c|}{ PWR $17 \times 17$} \\
\hline$\underline{B U^{(a)}}$ & PLHR ${ }^{(b)}$ & BU & PLHR & BU & PLHR & BU & PLHR \\
\hline $\begin{array}{r}0 \\
0 \\
0 \\
0 \\
1 \\
1 \\
2 \\
13 \\
27 \\
55 \\
110 \\
192 \\
274 \\
356 \\
437 \\
518 \\
598 \\
678 \\
758 \\
837 \\
916 \\
995 \\
1073 \\
1151 \\
1229 \\
1306 \\
1382 \\
1459 \\
1535 \\
1611 \\
1686 \\
1761 \\
1836 \\
1910 \\
1985 \\
2058 \\
2132 \\
2205 \\
2277 \\
2350 \\
2422 \\
2493 \\
2565\end{array}$ & $\begin{array}{r}0.0 \\
6.6 \\
13.1 \\
19.7 \\
26.3 \\
31.2 \\
35.0 \\
35.0 \\
35.0 \\
35.0 \\
34.8 \\
34.7 \\
34.5 \\
34.4 \\
34.2 \\
34.1 \\
33.9 \\
33.8 \\
33.6 \\
33.4 \\
33.3 \\
33.1 \\
33.0 \\
32.8 \\
32.7 \\
32.5 \\
32.4 \\
32.2 \\
32.1 \\
31.9 \\
31.8 \\
31.6 \\
31.5 \\
31.4 \\
31.2 \\
31.1 \\
30.9 \\
30.8 \\
30.6 \\
30.5 \\
30.4 \\
30.2 \\
30.1\end{array}$ & $\begin{array}{r}0 \\
0 \\
0 \\
0 \\
1 \\
1 \\
3 \\
18 \\
37 \\
74 \\
149 \\
260 \\
371 \\
481 \\
590 \\
698 \\
806 \\
913 \\
1020 \\
1126 \\
1231 \\
1335 \\
1439 \\
1542 \\
1645 \\
1747 \\
1848 \\
1949 \\
2049 \\
2149 \\
2247 \\
2346 \\
2443 \\
2540\end{array}$ & $\begin{array}{r}0.0 \\
6.6 \\
13.1 \\
19.7 \\
26.3 \\
32.8 \\
35.0 \\
35.0 \\
35.0 \\
35.0 \\
34.8 \\
34.6 \\
34.4 \\
34.2 \\
33.9 \\
33.7 \\
33.5 \\
33.3 \\
33.1 \\
32.9 \\
32.7 \\
32.5 \\
32.3 \\
32.1 \\
31.9 \\
31.7 \\
31.5 \\
31.3 \\
31.1 \\
30.9 \\
30.7 \\
30.5 \\
30.3 \\
30.2\end{array}$ & $\begin{array}{r}0 \\
0 \\
0 \\
0 \\
1 \\
1 \\
4 \\
23 \\
47 \\
96 \\
192 \\
287 \\
383 \\
478 \\
572 \\
666 \\
760 \\
853 \\
946 \\
1039 \\
1131 \\
1223 \\
1314 \\
1405 \\
1496 \\
1586 \\
1676 \\
1765 \\
1854 \\
1943 \\
2031 \\
2119 \\
2207 \\
2294 \\
2381 \\
2467 \\
2553\end{array}$ & $\begin{array}{r}0.0 \\
6.6 \\
13.1 \\
19.7 \\
26.3 \\
32.8 \\
35.0 \\
35.0 \\
35.0 \\
34.9 \\
34.8 \\
34.7 \\
34.5 \\
34.4 \\
34.2 \\
34.1 \\
33.9 \\
33.8 \\
33.6 \\
33.5 \\
33.3 \\
33.2 \\
33.1 \\
32.9 \\
32.8 \\
32.6 \\
32.5 \\
32.4 \\
32.2 \\
32.1 \\
32.0 \\
31.8 \\
31.7 \\
31.5 \\
31.4 \\
31.3 \\
31.1\end{array}$ & $\begin{array}{r}0 \\
0 \\
0 \\
0 \\
1 \\
2 \\
5 \\
30 \\
61 \\
123 \\
247 \\
370 \\
450 \\
615 \\
736 \\
856 \\
975 \\
1094 \\
1212 \\
1330 \\
1447 \\
1563 \\
1678 \\
1793 \\
1907 \\
2021 \\
2133 \\
2246 \\
2357 \\
2468 \\
2578\end{array}$ & $\begin{array}{r}0.0 \\
6.6 \\
13.1 \\
19.7 \\
26.3 \\
32.8 \\
35.0 \\
35.0 \\
35.0 \\
34.9 \\
34.7 \\
34.5 \\
34.3 \\
34.1 \\
33.9 \\
33.7 \\
33.5 \\
33.3 \\
33.1 \\
32.9 \\
32.7 \\
32.6 \\
32.4 \\
32.2 \\
32.0 \\
31.8 \\
31.6 \\
31.4 \\
31.2 \\
31.0 \\
30.9\end{array}$ \\
\hline
\end{tabular}

(a) $\mathrm{BU}=$ burnup in $\mathrm{GJ} / \mathrm{kgU}$

(b) $P L H R=$ peak linear heat rate in $\mathrm{kW} / \mathrm{m}$ 
TABLE 4. Assumed Input Uncertainties Common to All Rod Types

Local linear heat rate $(q)$

Coolant temperature $\left(T_{W}\right)$

Fuel diameter $\left(d_{f i}\right)$

Cladding inner diameter $\left(d_{c i}\right)$

$C l$ adding outer diameter $\left(d_{C O}\right)$

Fuel and cladding roughness $(R)$

Cladding thermal conductivity $\left(K_{C}\right)$

Fuel thermal conductivity $\left(K_{F}\right)$

Fuel specific heat $\left(C_{p}\right)$

Water film coefficient $\left(h_{f}\right)$

Contact conductance coefficients $\left(C_{2}, C_{3}\right)$

Temperature jump distance coefficient $\left(a_{g}\right)$

Fuel area function $\left(F_{p}\right)$

Fuel relocation parameter $\left(F_{p r}\right)$

Flux depression $(F)$

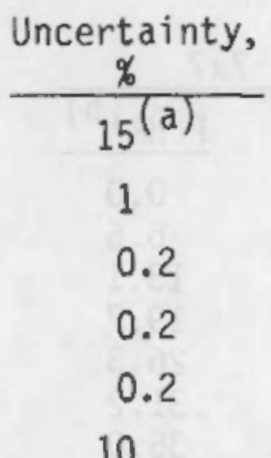

15

15

10

100

0.3

0.5

(c)

(a) All uncertainties are for a $3 \sigma$ confidence level.

(b) Varies linearly from $10 \%$ at $0 \mathrm{GJ} / \mathrm{kgU}$ to $20 \%$ at $432 \mathrm{GJ} / \mathrm{kgU}$.

(c) Varies linearly from $5 \%$ at $0 \mathrm{GJ} / \mathrm{kgU}$ to $10 \%$ at $432 \mathrm{GJ} / \mathrm{kgU}$. 
TABLE 5. Gas Thermal Conductivity Uncertainty, Varying Peak Power (Case 1)

\begin{tabular}{|c|c|c|c|c|c|c|c|c|}
\hline \multicolumn{2}{|c|}{ BWR $7 \times 7$} & \multicolumn{3}{|c|}{ BWR $8 \times 8$} & \multicolumn{2}{|c|}{ PWR $15 \times 15$} & \multicolumn{2}{|c|}{ PWR $17 \times 17$} \\
\hline$B U^{(a)}$ & $U K_{g}{ }^{(b)}$ & BU & $\mathrm{UK}_{\mathrm{g}}$ (1 atm) & $\mathrm{UK}_{\mathrm{g}}(3 \mathrm{~atm})$ & $\mathrm{BU}$ & $U K_{g}$ & $\mathrm{BU}$ & $\mathrm{UK}_{\mathrm{g}}$ \\
\hline 0 & 2.0 & & & & & & & \\
\hline 0 & 2.0 & 0 & 2.0 & 2.0 & 0 & 2.0 & 0 & 2.0 \\
\hline 0 & 2.0 & 0 & 2.0 & 2.0 & 0 & 2.0 & 0 & 2.0 \\
\hline 0 & 2.0 & 0 & 2.0 & 2.0 & 0 & 2.0 & 0 & 2.0 \\
\hline 1 & 2.0 & 0 & 2.0 & 2.0 & 1 & 2.0 & 1 & 2.0 \\
\hline 2 & 2.0 & 1 & 2.0 & 2.0 & 1 & 2.0 & 1 & 2.0 \\
\hline 13 & 2.0 & 1 & 2.0 & 2.0 & 1 & 2.0 & 4 & 2.0 \\
\hline 26 & 2.0 & 3 & 2.0 & 2.0 & 4 & 2.0 & 22 & 2.0 \\
\hline 53 & 2.0 & 15 & 2.0 & 2.0 & 21 & 2.0 & 44 & 2.0 \\
\hline 107 & 3.4 & 31 & 2.0 & 2.0 & 43 & 2.0 & 89 & 2.0 \\
\hline 187 & 6.2 & 62 & 2.3 & 2.0 & 88 & 2.0 & 177 & 2.0 \\
\hline 266 & 9.3 & 124 & 3.5 & 2.0 & 175 & 2.0 & 266 & 2.0 \\
\hline 346 & 12.7 & 216 & 7.7 & 2.8 & 263 & 2.0 & 353 & 2.0 \\
\hline 425 & 16.3 & 308 & 11.1 & 4.2 & 350 & 2.0 & 441 & 2.0 \\
\hline 503 & 20.4 & 399 & 14.8 & 5.7 & 437 & 2.0 & 528 & 2.0 \\
\hline 581 & 24.7 & 490 & 18.5 & 7.3 & 524 & 2.0 & 615 & 2.3 \\
\hline 659 & 28.0 & 581 & 22.2 & 8.9 & 610 & 2.0 & 702 & 2.7 \\
\hline 737 & 29.4 & 671 & 26.4 & 10.7 & 696 & 2.2 & 788 & 3.2 \\
\hline 814 & 30.2 & 760 & 30.7 & 12.5 & 782 & 2.6 & 874 & 3.7 \\
\hline 891 & 30.6 & 849 & 35.0 & 14.3 & 867 & 3.0 & 959 & 4.2 \\
\hline 967 & 30.7 & 938 & 39.8 & 16.3 & 952 & 3.4 & 1044 & 4.7 \\
\hline 1043 & 30.8 & 1026 & 44.7 & 18.4 & 1036 & 3.8 & 1129 & 5.3 \\
\hline 1119 & 30.4 & 1114 & 49.8 & 20.5 & 1121 & 4.3 & 1213 & 5.8 \\
\hline 1195 & 30.0 & 1201 & 55.2 & 22.6 & 1205 & 4.8 & 1297 & 6.2 \\
\hline 1270 & 29.4 & 1288 & 55.1 & 23.5 & 1288 & 5.0 & 1381 & 6.5 \\
\hline 1345 & 28.8 & 1375 & 54.4 & 24.3 & 1372 & 5.4 & 1464 & 6.8 \\
\hline 1419 & 28.1 & 1460 & 54.3 & 25.2 & 1455 & 5.6 & 1547 & 7.2 \\
\hline 1493 & 27.3 & 1546 & 53.0 & 25.7 & 1537 & 5.9 & 1629 & 7.5 \\
\hline 1567 & 26.5 & 1631 & 51.5 & 26.1 & 1620 & 6.2 & 1712 & 7.8 \\
\hline 1639 & 25.6 & 1716 & 50.5 & 26.6 & 1702 & 6.4 & 1794 & 8.1 \\
\hline 1714 & 24.9 & 1800 & 48.1 & 26.6 & 1783 & 6.7 & 1875 & 8.3 \\
\hline 1786 & 23.8 & 1883 & 45.9 & 26.7 & 1865 & 6.9 & 1957 & 8.5 \\
\hline 1859 & 22.7 & 1967 & 42.5 & 26.2 & 1946 & 7.1 & 2038 & 8.7 \\
\hline 1931 & 21.5 & 2050 & 38.7 & 25.4 & 2026 & 7.3 & 2087 & 8.8 \\
\hline 2003 & 19.9 & 2132 & 35.0 & 24.5 & 2107 & 7.4 & 2198 & 9.0 \\
\hline 2074 & 18.3 & 2214 & 31.6 & 23.4 & 2187 & 7.6 & 2278 & 9.0 \\
\hline 2146 & 16.8 & 2296 & 28.2 & 22.2 & 2267 & 7.6 & 2358 & 9.0 \\
\hline 2217 & 15.3 & 2377 & 25.1 & 20.9 & 2345 & 7.7 & 2437 & 9.0 \\
\hline 2283 & 13.9 & 2459 & 22.2 & 19.5 & 2425 & 7.7 & 2516 & 8.9 \\
\hline 2358 & 12.6 & 2538 & 19.5 & 18.1 & 2504 & 7.7 & 2595 & 8.8 \\
\hline & & & & & 2583 & 7.7 & & \\
\hline
\end{tabular}

(a) $8 \mathrm{U}=$ burnup in $\mathrm{GJ} / \mathrm{kgU}$

(b) $\mathrm{UK}_{\mathrm{g}}=$ gas thermal conductivity relative uncertainty, $\%$ 
TABLE 6. Gas Thermal Conductivity Uncertainty, $35 \mathrm{~kW} / \mathrm{m}$ Peak Power (Case 2)

\begin{tabular}{|c|c|c|c|c|}
\hline \multicolumn{2}{|c|}{ BWR $7 \times 7$} & \multicolumn{3}{|c|}{ BUR $8 \times 8$} \\
\hline $8 u^{(a)}$ & $\mathrm{UK}_{\mathrm{g}}{ }^{(b)}$ & BU & $\mathrm{UK}_{\mathrm{g}}(1 \mathrm{~atm})$ & $U \mathrm{~K}_{\mathrm{g}}$ (3 atm) \\
\hline 0 & 2.0 & 0 & 2.0 & 2.0 \\
\hline 0 & 2.0 & 0 & 2.0 & 2.0 \\
\hline 0 & 2.0 & 0 & 2.0 & 2.0 \\
\hline 0 & 2.0 & 0 & 2.0 & 2.0 \\
\hline 1 & 2.0 & 1 & 2.0 & 2.0 \\
\hline 1 & 2.0 & 1 & 2.0 & 2.0 \\
\hline 2 & 2.0 & 3 & 2.0 & 2.0 \\
\hline 13 & 2.0 & 18 & 2.0 & 2.0 \\
\hline 27 & 2.0 & 37 & 2.0 & 2.0 \\
\hline 55 & 2.0 & 74 & 2.8 & 2.0 \\
\hline 110 & 3.4 & 149 & 5.2 & 2.0 \\
\hline 192 & 6.4 & 260 & 9.4 & 3.4 \\
\hline 274 & 9.6 & 371 & 13.7 & 5.2 \\
\hline 356 & 13.1 & 481 & 18.1 & 7.1 \\
\hline 437 & 17.0 & 590 & 22.8 & 9.1 \\
\hline 518 & 21.2 & 698 & 27.7 & 11.2 \\
\hline 598 & 25.6 & 806 & 32.9 & 13.4 \\
\hline 678 & 28.4 & 913 & 38.4 & 15.7 \\
\hline 758 & 29.4 & 1020 & 44.2 & 18.1 \\
\hline 837 & 30.3 & 1126 & 50.5 & 20.7 \\
\hline 916 & 30.7 & 1231 & 55.5 & 23.0 \\
\hline 995 & 30.8 & 1335 & 54.7 & 23.9 \\
\hline 1073 & 30.6 & 1439 & 54.0 & 24.8 \\
\hline 1151 & 30.2 & 1542 & 52.9 & 25.6 \\
\hline 1229 & 29.7 & 1645 & 51.4 & 26.1 \\
\hline 1306 & 29.0 & 1747 & 49.8 & 26.6 \\
\hline 1382 & 28.4 & 1848 & 46.9 & 26.7 \\
\hline 1459 & 27.6 & 1949 & 43.2 & 26.3 \\
\hline 1535 & 26.9 & 2049 & 38.7 & 25.4 \\
\hline 1611 & 26.0 & 2149 & 34.3 & 24.3 \\
\hline 1686 & 25.2 & 2247 & 30.2 & 22.9 \\
\hline 1761 & 24.2 & 2346 & 26.3 & 21.4 \\
\hline 1836 & 23.1 & 2443 & 22.7 & 19.8 \\
\hline 1910 & 21.8 & 2540 & 19.4 & 18.1 \\
\hline 1985 & 20.3 & & & \\
\hline 2063 & 18.7 & & & \\
\hline 2132 & 17.1 & & & \\
\hline 2205 & 15.6 & & in & \\
\hline 2277 & 14.1 & & & \\
\hline 2350 & 12.8 & & & \\
\hline 2422 & 11.5 & & & \\
\hline $\begin{array}{l}2493 \\
2565\end{array}$ & 10.3 & & & \\
\hline 2565 & 9.2 & & & \\
\hline
\end{tabular}
PUR $15 \times 15$

\begin{tabular}{|c|c|c|c|c|c|}
\hline BU & $\mathrm{UKg}_{\mathrm{g}}$ & BU & $\mathrm{UK}_{\mathrm{g}}(20 \mathrm{~atm})$ & BU & $\mathrm{UK}_{\mathrm{g}}$ (1 atm) \\
\hline $\begin{array}{r}0 \\
0 \\
0 \\
1 \\
1 \\
1 \\
4 \\
23 \\
47 \\
96 \\
192 \\
287 \\
383 \\
478 \\
572 \\
666 \\
760 \\
853 \\
946 \\
1039 \\
1131 \\
1223 \\
1314 \\
1405 \\
1496 \\
1586 \\
1676 \\
1765 \\
1854 \\
1943 \\
2031 \\
2119 \\
2207 \\
2294 \\
2381 \\
2467 \\
2553\end{array}$ & $\begin{array}{l}2.0 \\
2.0 \\
2.0 \\
2.0 \\
2.0 \\
2.0 \\
2.0 \\
2.0 \\
2.0 \\
2.0 \\
2.0 \\
2.0 \\
2.0 \\
2.0 \\
2.0 \\
2.1 \\
2.5 \\
2.9 \\
3.4 \\
3.9 \\
4.4 \\
4.8 \\
5.1 \\
5.5 \\
5.8 \\
6.1 \\
6.3 \\
6.6 \\
6.9 \\
7.1 \\
7.3 \\
7.4 \\
7.6 \\
7.7 \\
7.7 \\
7.7 \\
7.7\end{array}$ & $\begin{array}{r}0 \\
0 \\
0 \\
1 \\
1 \\
2 \\
5 \\
30 \\
61 \\
123 \\
247 \\
370 \\
450 \\
615 \\
736 \\
856 \\
975 \\
1094 \\
1212 \\
1330 \\
1447 \\
1563 \\
1678 \\
1793 \\
1907 \\
2021 \\
2133 \\
2246 \\
2357 \\
2468 \\
2578\end{array}$ & $\begin{array}{l}2.0 \\
2.0 \\
2.0 \\
2.0 \\
2.0 \\
2.0 \\
2.0 \\
2.0 \\
2.0 \\
2.0 \\
2.0 \\
2.0 \\
2.0 \\
2.3 \\
2.9 \\
3.6 \\
4.3 \\
5.1 \\
5.8 \\
6.3 \\
6.8 \\
7.2 \\
7.7 \\
8.1 \\
8.4 \\
8.7 \\
8.9 \\
9.0 \\
9.0 \\
8.9 \\
8.8\end{array}$ & $\begin{array}{r}0 \\
0 \\
0 \\
1 \\
1 \\
2 \\
3 \\
10 \\
16 \\
28 \\
41 \\
59 \\
91 \\
122 \\
184 \\
276 \\
369 \\
460 \\
552 \\
643 \\
733 \\
824 \\
914 \\
1003 \\
1092 \\
1181 \\
1269 \\
1357 \\
1444 \\
1531 \\
1618 \\
1704 \\
1790 \\
1876 \\
1961 \\
2046 \\
2131 \\
2215 \\
2298 \\
2382 \\
2465 \\
2547\end{array}$ & $\begin{array}{r}2.0 \\
2.0 \\
2.0 \\
2.0 \\
2.0 \\
2.0 \\
2.0 \\
2.0 \\
2.0 \\
2.0 \\
2.0 \\
2.3 \\
3.8 \\
5.2 \\
8.5 \\
13.0 \\
17.7 \\
22.7 \\
27.6 \\
32.8 \\
38.5 \\
44.4 \\
50.8 \\
57.5 \\
64.5 \\
71.9 \\
72.7 \\
70.3 \\
67.6 \\
64.6 \\
61.5 \\
59.2 \\
55.5 \\
51.5 \\
46.5 \\
41.3 \\
36.3 \\
32.0 \\
27.8 \\
24.1 \\
20.8 \\
17.7\end{array}$ \\
\hline
\end{tabular}

(a) $\mathrm{BU}=$ burnup in $\mathrm{GJ} / \mathrm{kgU}$

(b) $\mathrm{UK}_{g}=$ gas thermal conductivity relative uncertainty, $\%$ 
TABLE 7. Uncertainty for Water Film and Cladding Temperatures at $35 \mathrm{~kW} / \mathrm{m}$

\begin{tabular}{|c|c|c|c|c|c|}
\hline \multirow{2}{*}{\multicolumn{2}{|c|}{ Rod Type }} & \multicolumn{3}{|c|}{$\begin{array}{c}\text { Relative } \\
\text { Uncertainty, } \%(3 \sigma)\end{array}$} & \multirow{2}{*}{$\begin{array}{c}\begin{array}{c}\text { Aosolute } \\
\text { Uncertainty, }\end{array} \\
{ }_{c^{5} \pm 3 \sigma}\end{array}$} \\
\hline & & $\Delta \mathrm{T}_{F}$ & $\Delta T_{C}$ & $T_{c}^{\xi}$ & \\
\hline BWR & $7 \times 7$ & 21.2 & 21.3 & 3.2 & $606 \pm 19.1$ \\
\hline$B W R$ & $8 \times 8$ & 21.2 & 21.3 & 3.7 & $017 \pm 22.7$ \\
\hline PWR & $15 \times 15$ & 21.2 & 21.3 & 3.2 & $634 \pm 20.0$ \\
\hline PWR & $17 x+7$ & 21.2 & 21.3 & 3.2 & $030 \pm 20.0$ \\
\hline
\end{tabular}

- For all rod types, the relative uncertainty for the cladding inner surface temperature is low $(<4 \%)$.

- All rod types have the same relative uncertainty for the water film temperature rise because relative uncertainty depends only upon the sum of the variances of the parameters that are used to calculate the temperature rise and those parameters are assumed to be the same for all rod types.

- All rod types have the same relative uncertainty for the cladding temperature rise because the relative uncertainty depends principally on the uncertainty to the input parameters, rather than the value of the parameters.

- For botn tne water film and cladding temperature rises, the temperatures are power dependent; but their relative uncertainties are not. The relative uncertainty for the cladding inner surface temperature has a slight power dependency because the uncertainty equation includes the power-dependent temperatures.

The uncertainty of the cladding inner surface temperature will not receive further attention in this analysis because it is low--neariy constant between rod types--and unchanging with burnup. 
Figures $2-12^{(a)}$ visually summarize the rod type analysis. Each figure includes as a function of burnup ${ }^{(b)}$ the GT3 predictions of fuel surface temperature, fuel centerline temperature, and stored energy above coolant, (c) along with the absolute uncertainty bounds $(d)$ ( $3 \sigma$ confidence level) as predicted by STORE. These figures illustrate the effect of design differences; from them, certain deductions can be made.

\section{FUEL ROD TEMPERATURES}

Beginning with the predicted thermal response of the fuel rod designs, there is a difference between the peak centerline temperatures of the different fuel rod designs at $\mathrm{BOL}$. At $35 \mathrm{~kW} / \mathrm{m}$, those temperatures are

$\begin{array}{ll}\text { BWR } 7 \times 7 & 1746 \mathrm{~K} \\ \text { BWR } 8 \times 8(1 \mathrm{~atm}) & 1696 \mathrm{~K} \\ \text { BWR } 8 \times 8(3 \mathrm{~atm}) & 1681 \mathrm{~K} \\ \text { PWR } 15 \times 15 & 1661 \mathrm{~K} \\ \text { PWR } 17 \times 17 & 1654 \mathrm{~K}\end{array}$

The differences in peak temperature are directly associated with the initial gap size. The BWR $7 \times 7$ has the largest gap and the highest temperature, and the PWR $17 \times 17$ has the smallest gap and the lowest temperature. The 3-atm BWR $8 \times 8$ rod has a slightly lower temperature than the 1-atm rod because the extra helium provides some improvement in gap conductance.

The most obvious feature of Figures $2-12$ is the rapid drop in temperature early in life. This drop is due to the relocation model in GT3 that specifies the change in the radial gap $\left(\Delta \mathrm{d}_{g}\right)$ as a function of power $(q)$ and burnup (BU):

(a) These figures begin with the first full power step after the rise to power.

(b) Tables 8 and 9 relate the burnup in the figures to elapsed time of irradiation for each rod design.

(c) For each rod type, the stored energy between 293K and coolant temperature is: $8 W R 7 \times 7=8.67 \times 10^{4} \mathrm{~J} / \mathrm{m}$, BWR $8 \times 8=6.39 \times 10^{4} \mathrm{~J} / \mathrm{m}$, PWR $15 \times 15=$ $5.44 \times 10^{4} \mathrm{~J} / \mathrm{m}$, PWR $17 \times 17=4.21 \times 10^{4} \mathrm{~J} / \mathrm{m}$. The conversion for $\mathrm{J} / \mathrm{m}$ to BTU/lb for each rod type is: BWR $7 \times 7=0.3466$, BWR $8 \times 8=0.4698$, PWR $15 \times 15=0.6117$, PWR $17 \times 17=0.7821$.

(d) These bounds are indicated on the figures by arrowheads. 

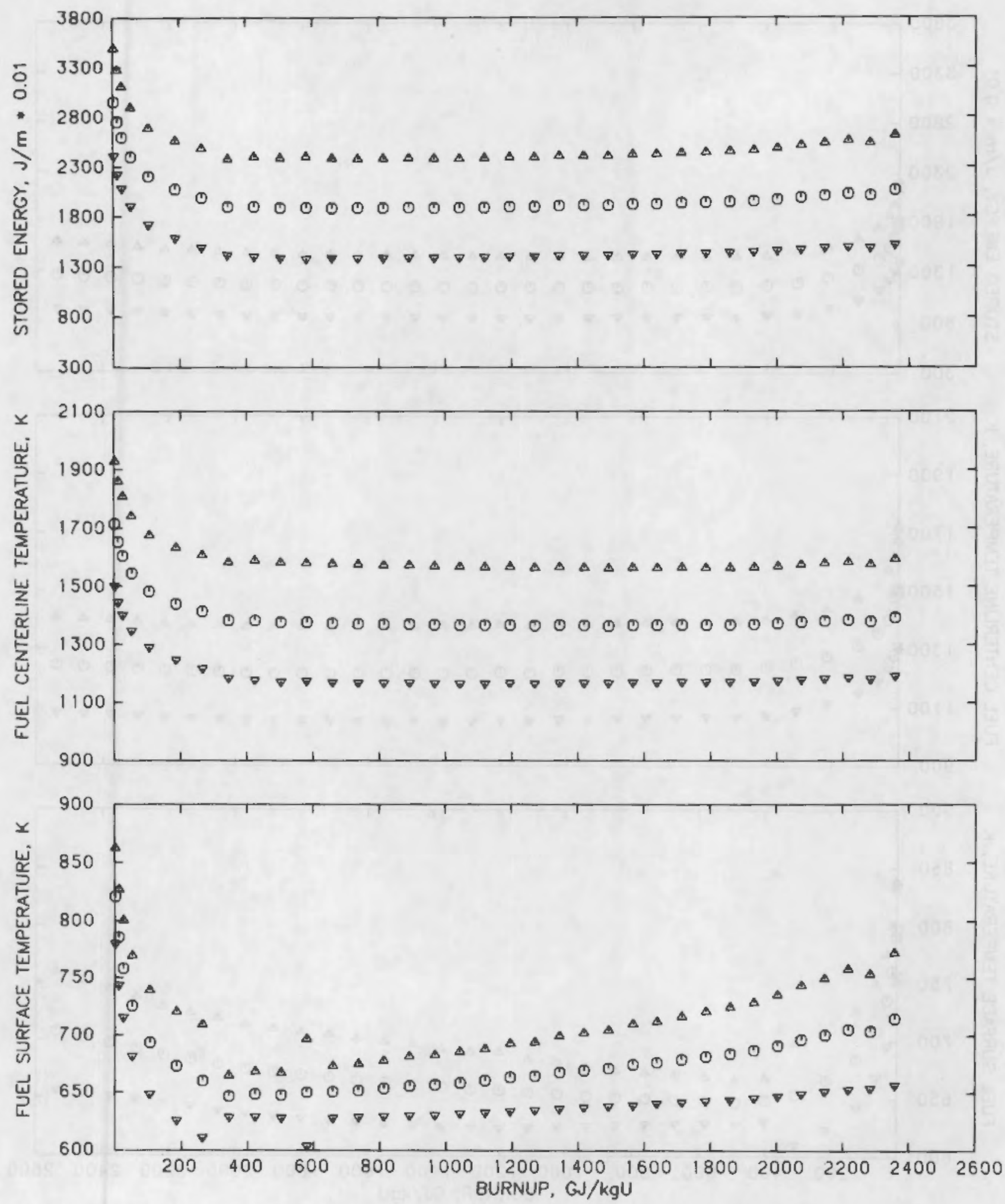

FIGURE 2. Thermal History for BWR $7 \times 7,34 \mathrm{~kW} / \mathrm{m}$ Peak Power 

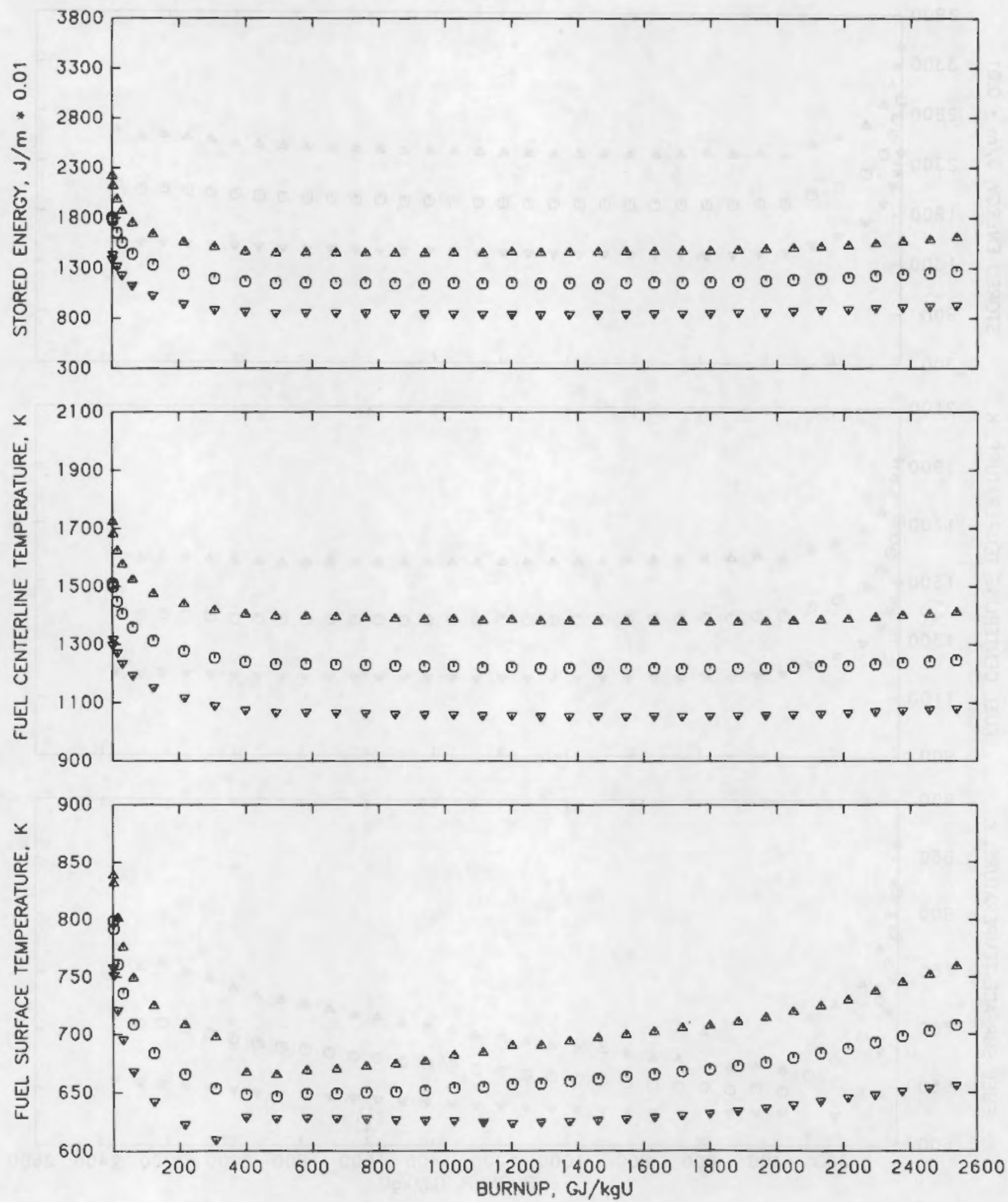

FIGURE 3. Thermal History for BWR $8 \times 8,29 \mathrm{~kW} / \mathrm{m}$ Peak Power 

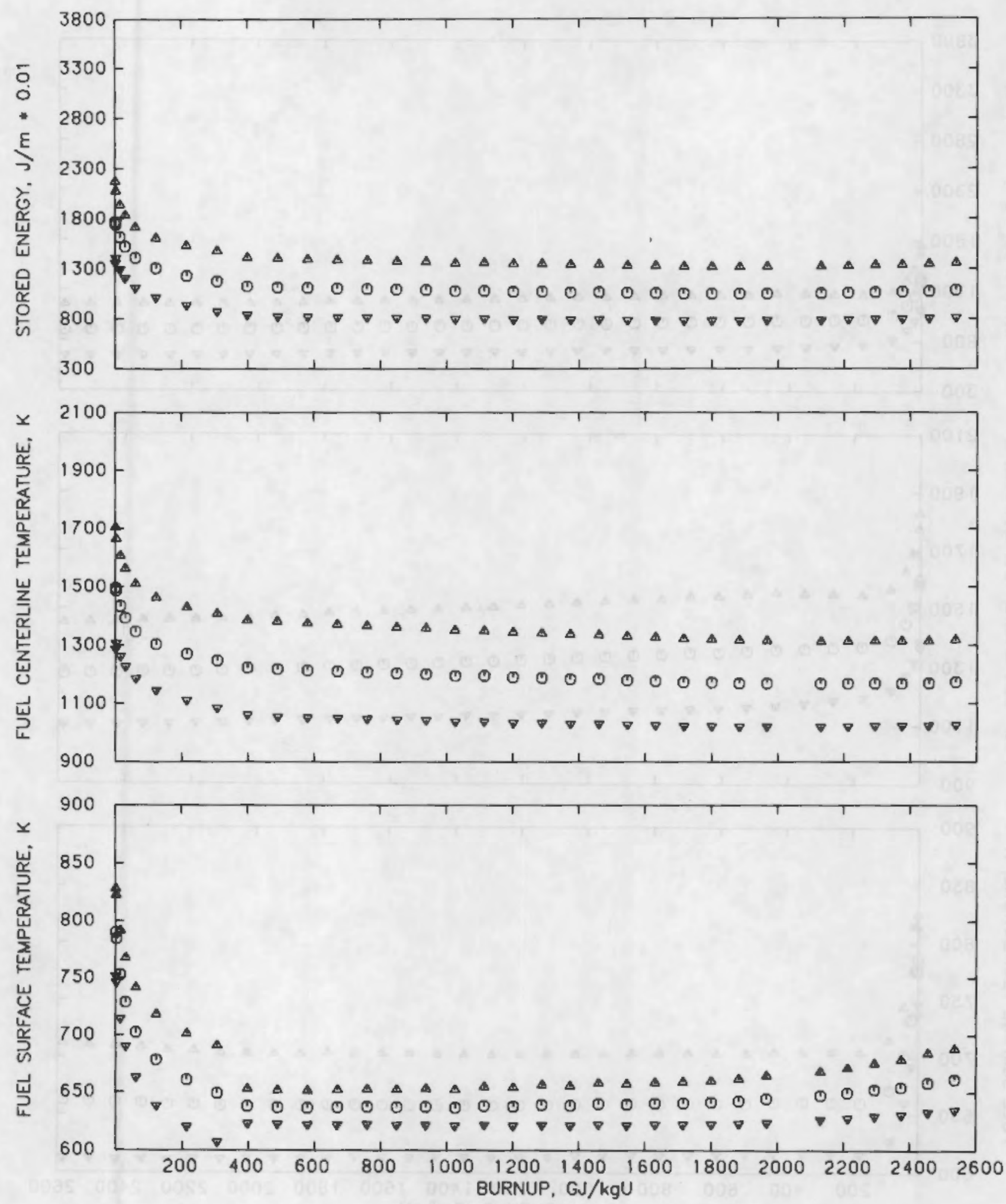

FIGURE 4. Thermal History for BWR $8 \times 8,3 \mathrm{~atm}, 29 \mathrm{~kW} / \mathrm{m}$ Peak Power 

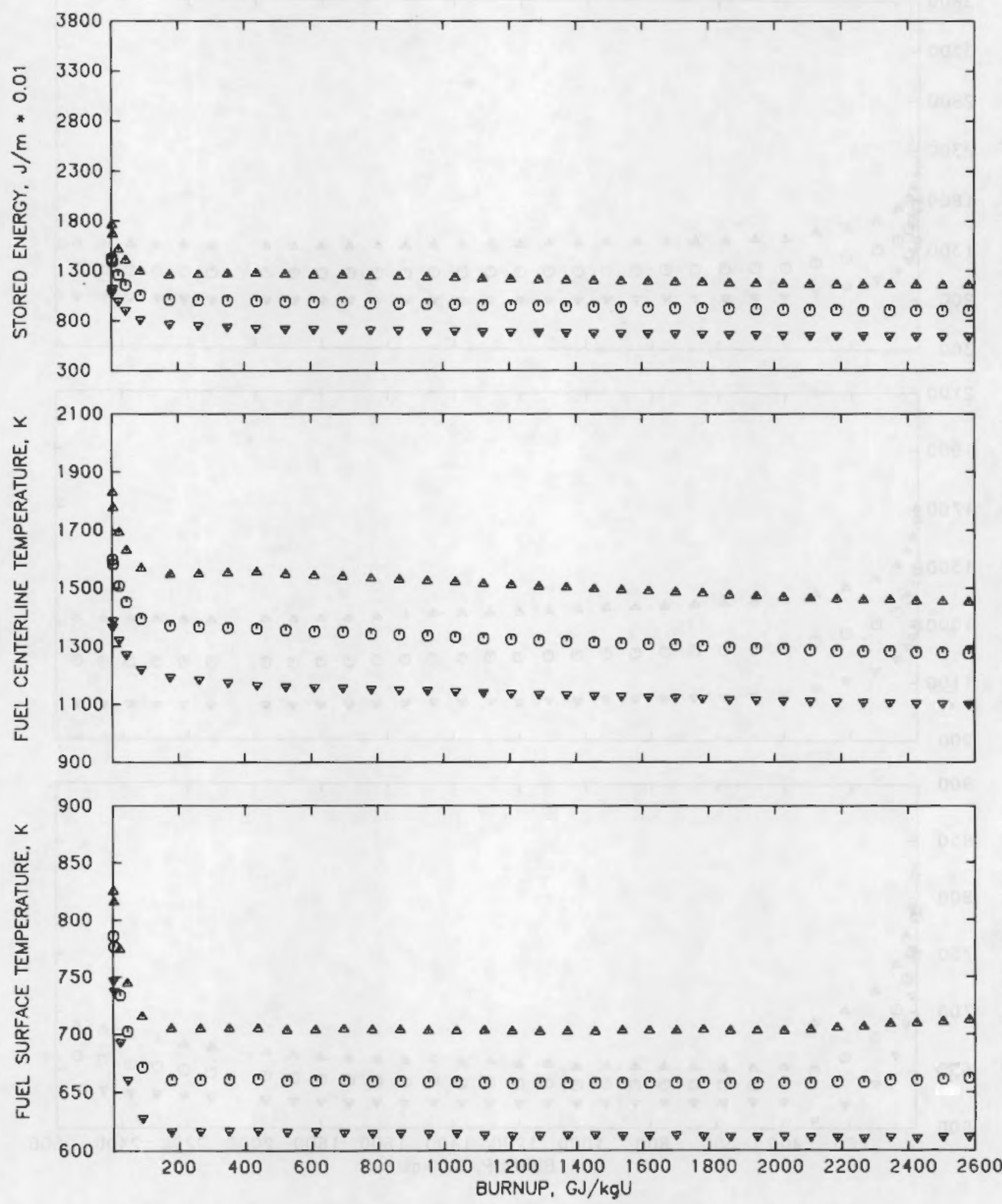

FIGURE 5. Thermal History for PWR $15 \times 15,32 \mathrm{~kW} / \mathrm{m}$ Peak Power 

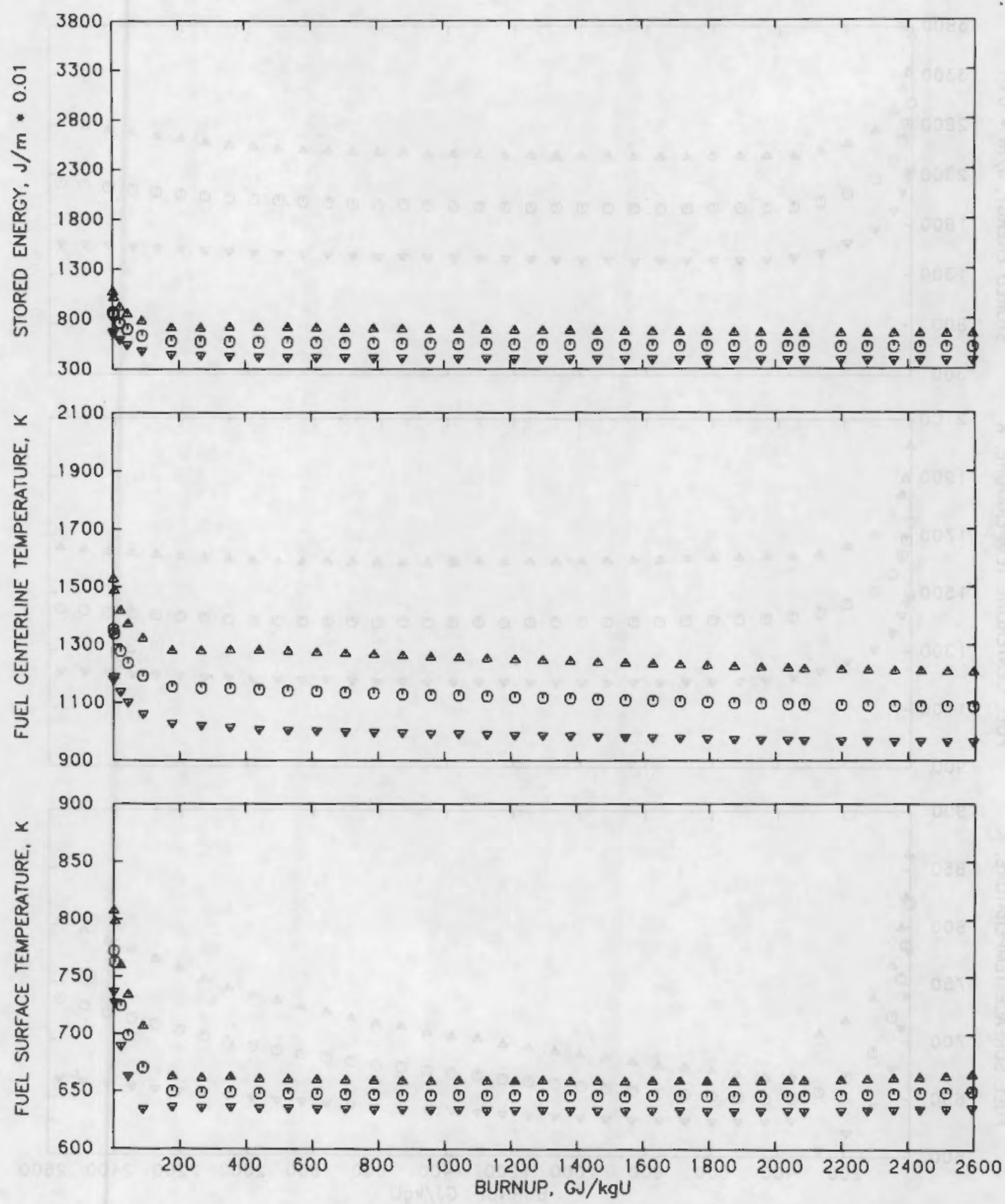

FIGURE 6. Thermal History for PWR $17 \times 17,25 \mathrm{~kW} / \mathrm{m}$ Peak Power 

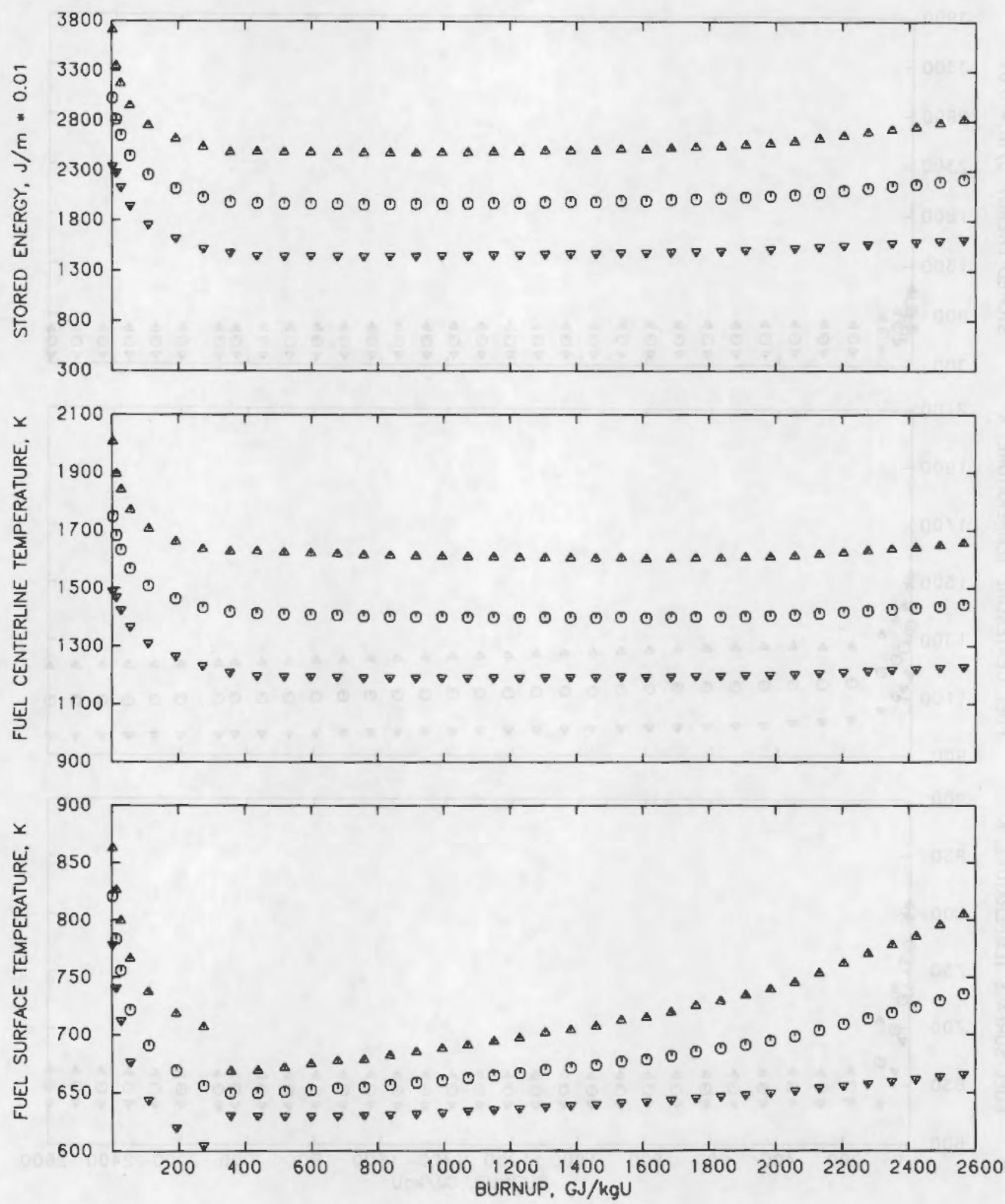

FIGURE 7. Thermal History for BWR $7 \times 7,35 \mathrm{~kW} / \mathrm{m}$ Peak Power 

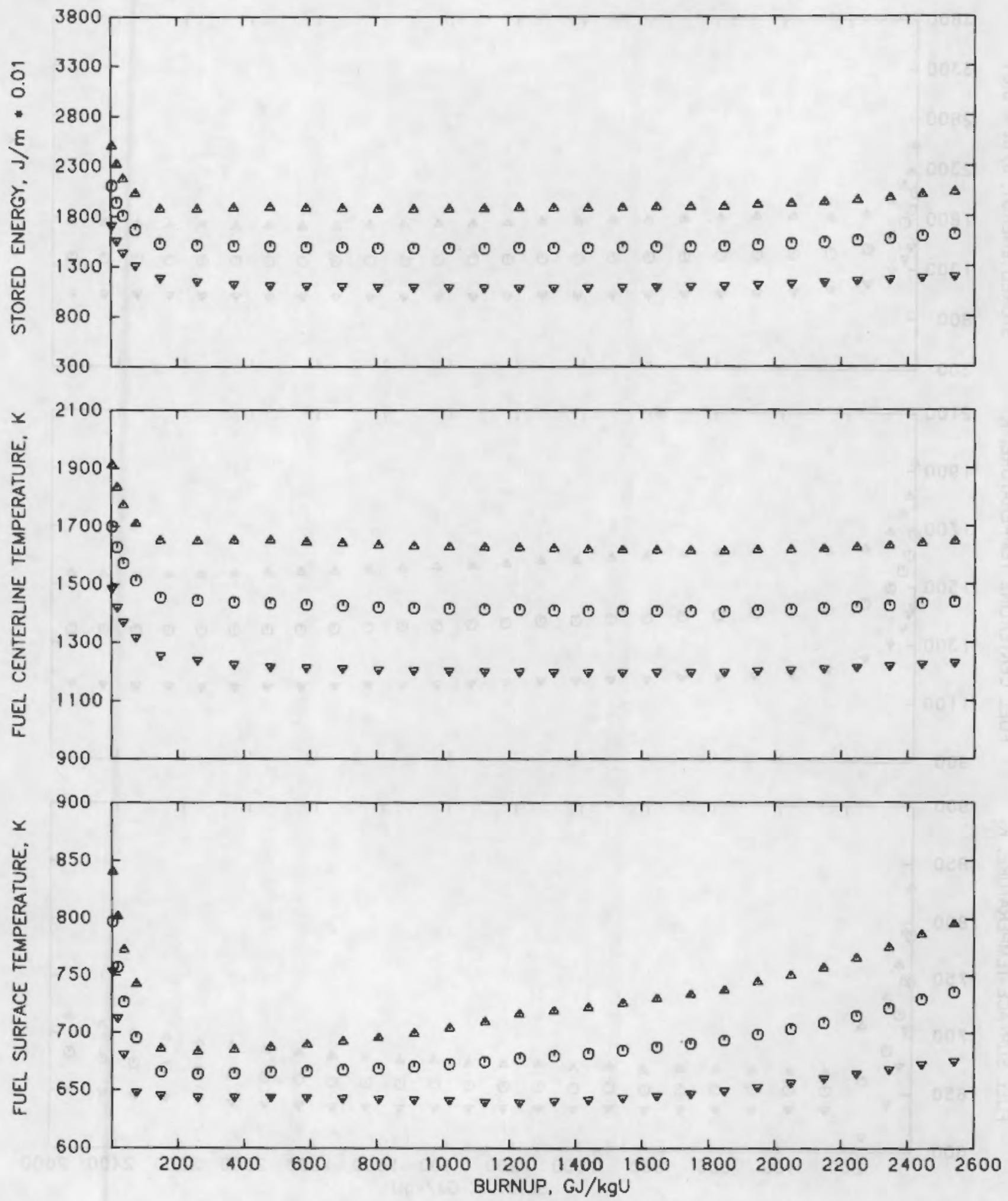

FIGURE 8. Thermal History for BWR $8 \times 8,35 \mathrm{~kW} / \mathrm{m}$ Peak Power 

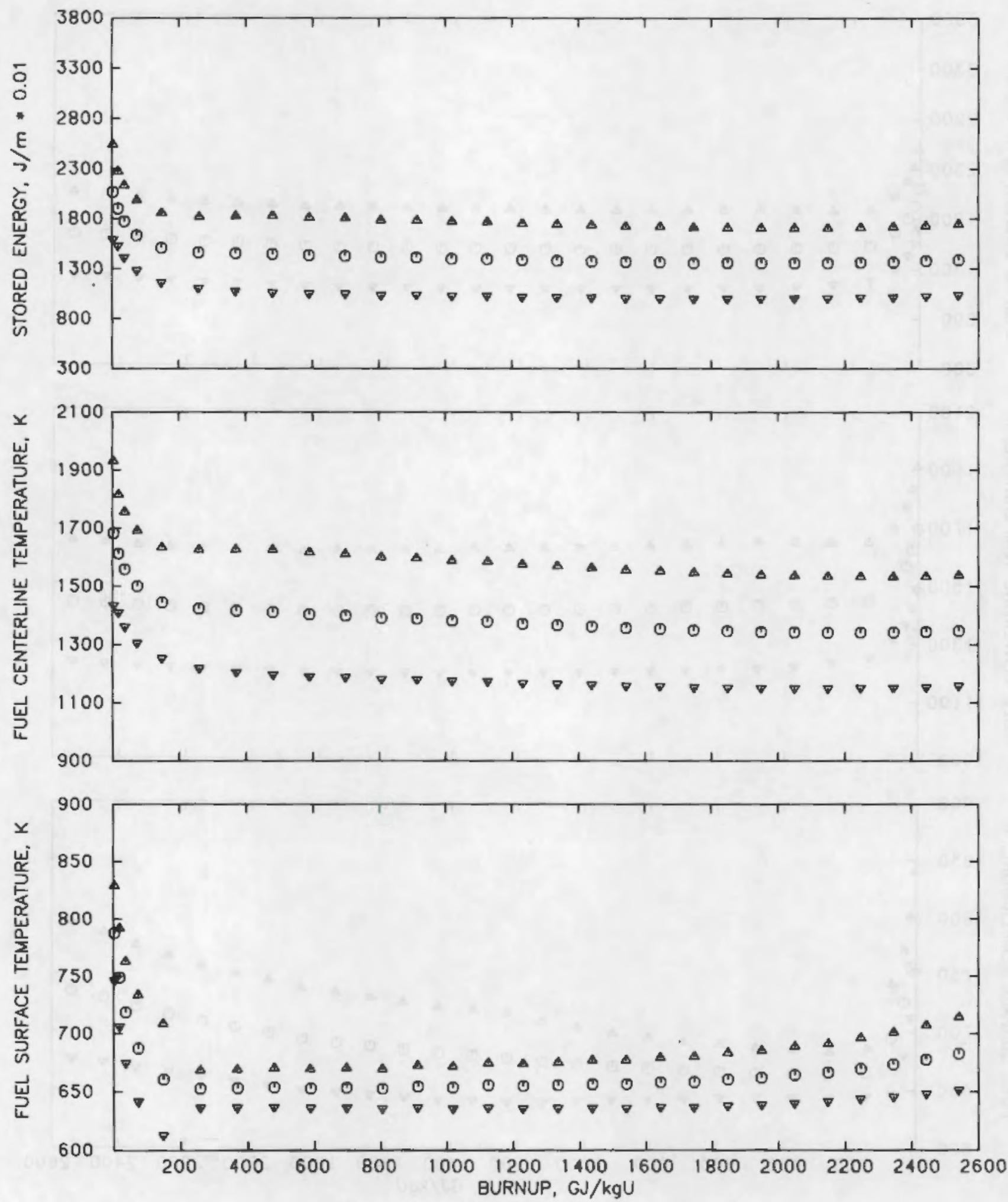

FIGURE 9. Thermal History for BWR $8 \times 8,3 \mathrm{~atm}, 35 \mathrm{~kW} / \mathrm{m}$ Peak Power 

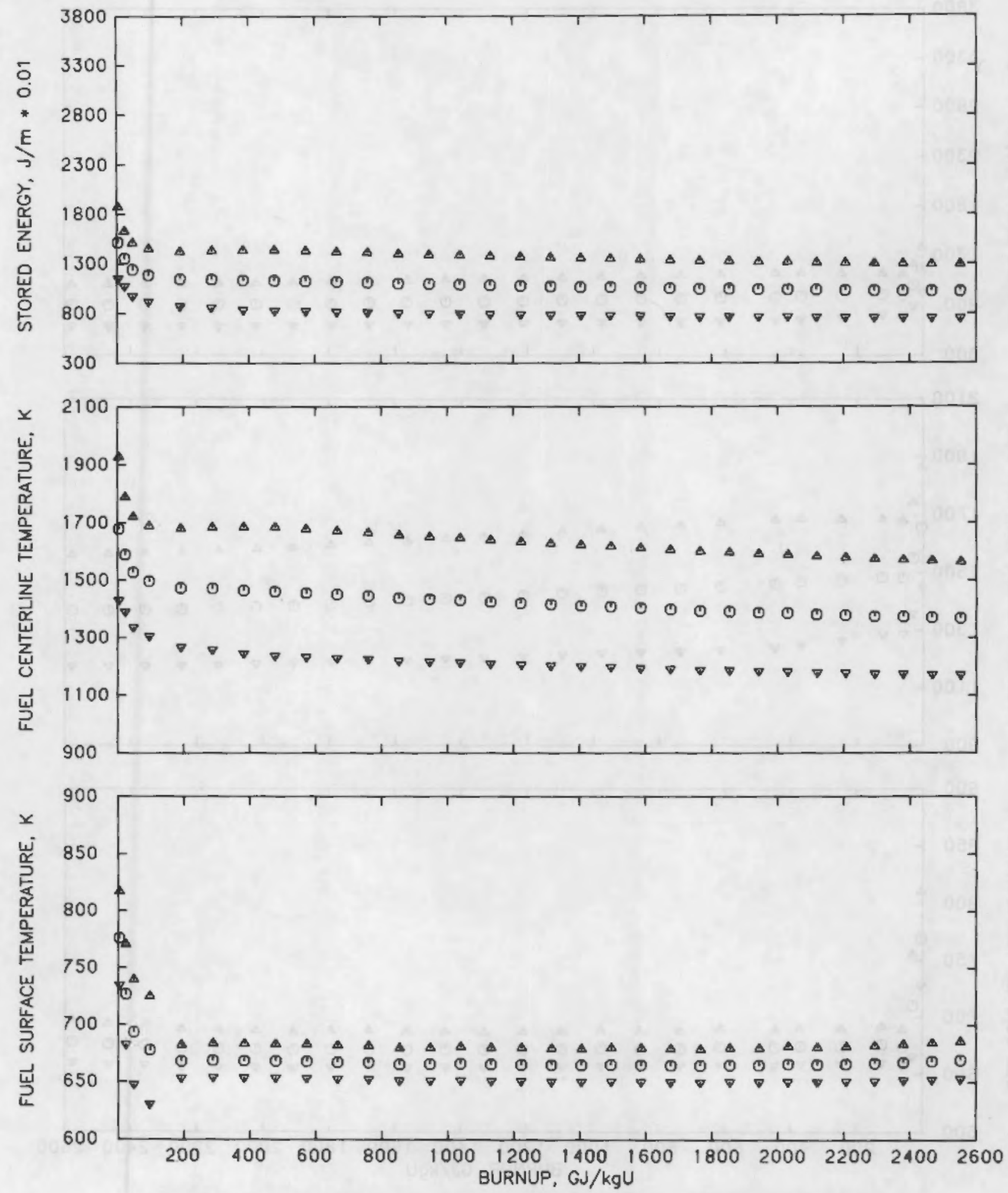

FIGURE 10. Thermal History for PWR $15 \times 15,35 \mathrm{~kW} / \mathrm{m}$ Peak Power 

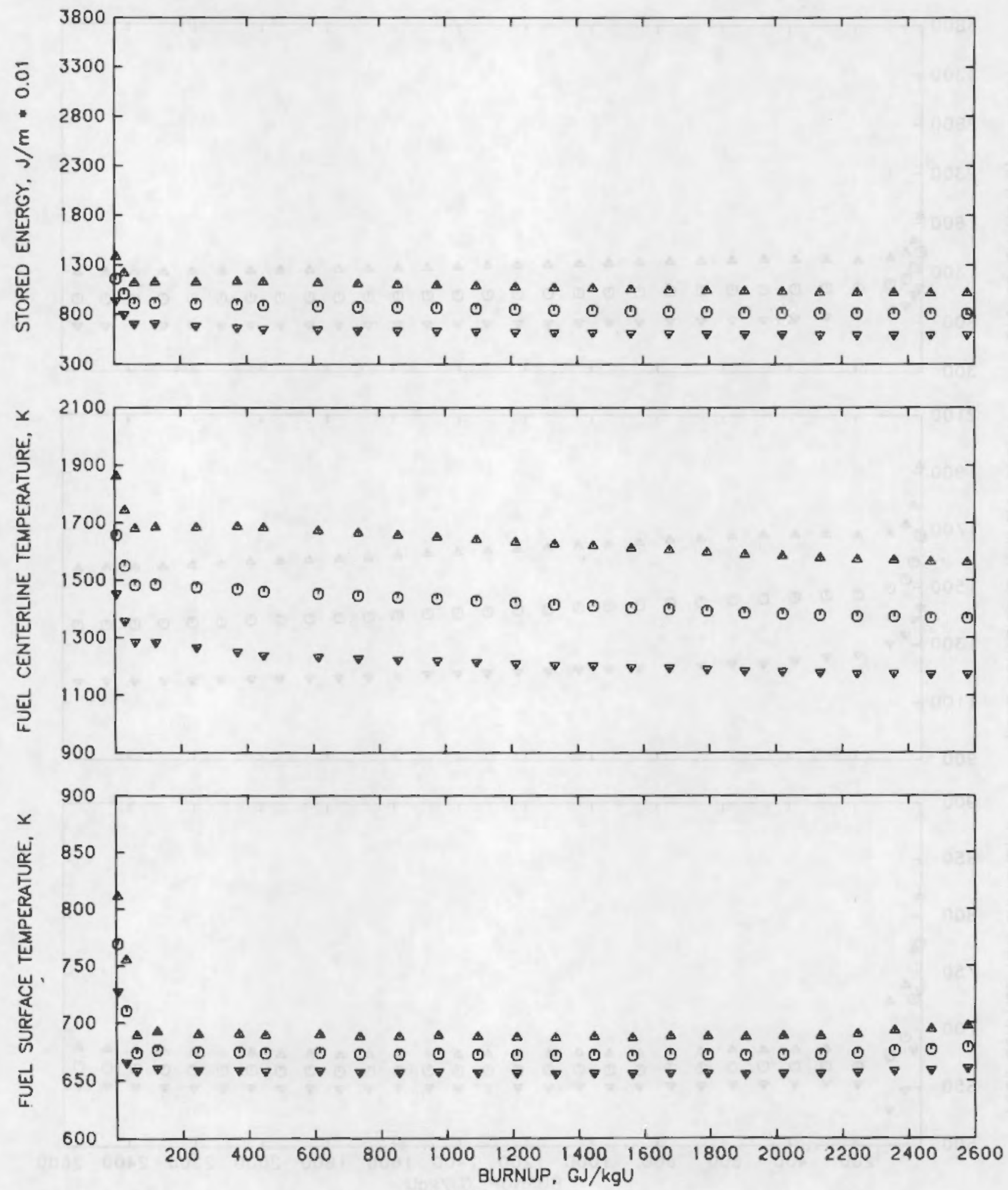

FIGURE 11. Thermal History for PWR $17 \times 17,35 \mathrm{~kW} / \mathrm{m}$ Peak Power 

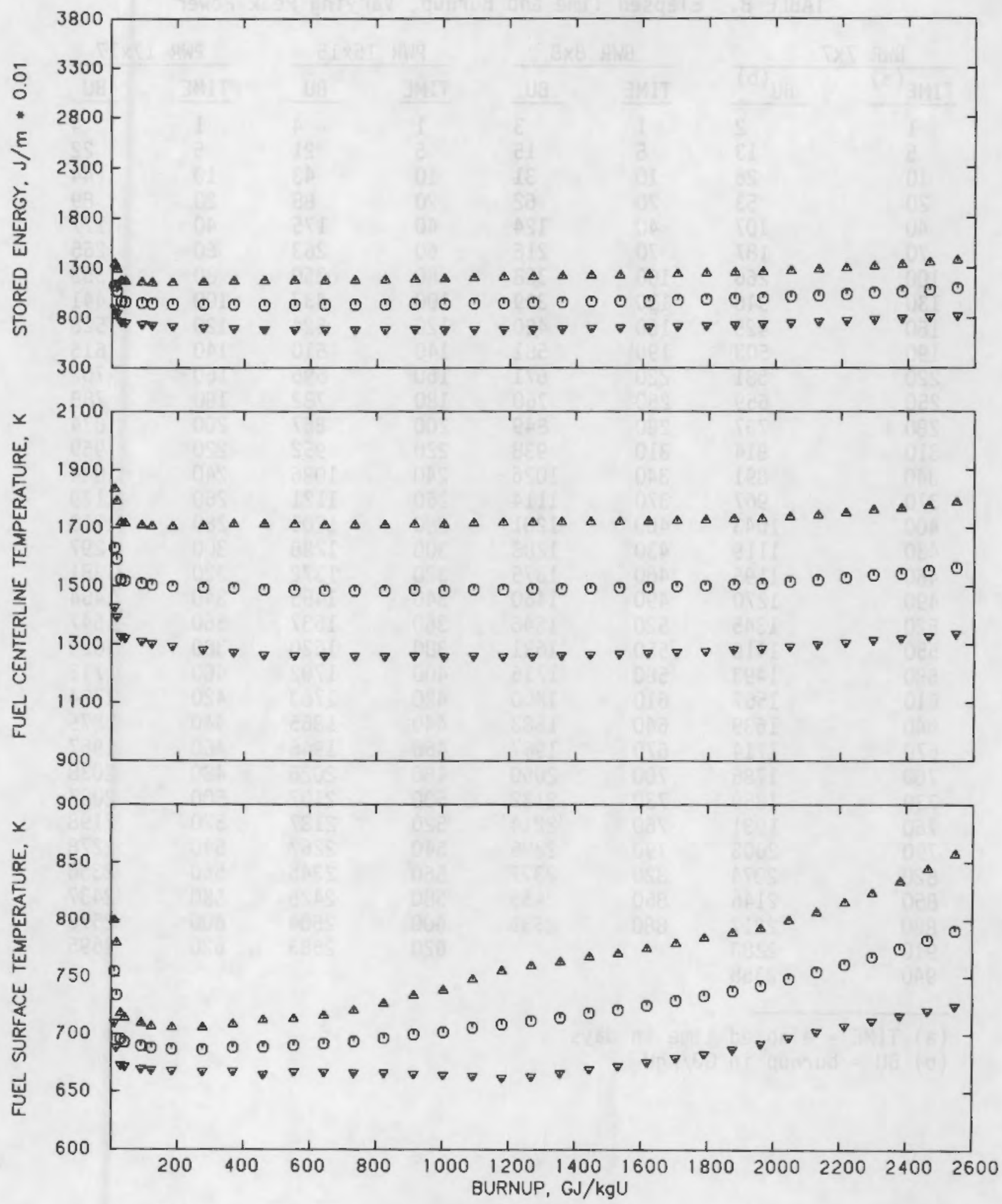

FIGURE 12. Thermal History for PWR $17 \times 17,1 \mathrm{~atm}, 35 \mathrm{~kW} / \mathrm{m}$ Peak Power 
TABLE B. Elapsed Time and Burnup, Varying Peak Power

\begin{tabular}{|c|c|c|c|c|c|c|c|}
\hline \multicolumn{2}{|c|}{ BWR $7 \times 7$} & \multicolumn{2}{|c|}{ BWR $8 \times 8$} & \multicolumn{2}{|c|}{ PWR $15 \times 15$} & \multicolumn{2}{|c|}{ PWR $17 \times 17$} \\
\hline $\operatorname{IIME}^{(\mathrm{a})}$ & $\mathrm{Bu}^{(b)}$ & TIME & BU & IIME & $B U$ & TIME & BU \\
\hline 1 & 2 & 1 & 3 & 1 & 4 & 1 & 4 \\
\hline 5 & 13 & 5 & 15 & 5 & 21 & 5 & 22 \\
\hline 10 & 26 & 10 & 31 & 10 & 43 & 10 & 44 \\
\hline 20 & 53 & 20 & 62 & 20 & 88 & 20 & 89 \\
\hline 40 & 107 & 40 & 124 & 40 & 175 & 40 & 177 \\
\hline 70 & 187 & 70 & 216 & 60 & 263 & 60 & 266 \\
\hline 100 & 266 & 100 & 308 & 80 & 350 & 80 & 353 \\
\hline 130 & 346 & 130 & 399 & 100 & 437 & 100 & 441 \\
\hline 160 & 425 & 160 & 490 & 120 & 524 & 120 & 528 \\
\hline 190 & 503 & 190 & 581 & 140 & 610 & 140 & 615 \\
\hline 220 & 581 & 220 & 671 & 160 & 696 & 160 & 702 \\
\hline 250 & 659 & 250 & 760 & 180 & 782 & 180 & 788 \\
\hline 280 & 737 & 280 & 849 & 200 & 867 & 200 & 874 \\
\hline 310 & 814 & 310 & 938 & 220 & 952 & 220 & 959 \\
\hline 340 & 891 & 340 & 1026 & 240 & 1036 & 240 & 1044 \\
\hline 370 & 967 & 370 & 1114 & 260 & 1121 & 260 & 1129 \\
\hline 400 & 1043 & 400 & 1201 & 280 & 1205 & 280 & 1213 \\
\hline 430 & 1119 & 430 & 1288 & 300 & 1288 & 300 & 1297 \\
\hline 460 & 1195 & 460 & 1375 & 320 & 1372 & 320 & 1381 \\
\hline 490 & 1270 & 490 & 1460 & 340 & 1455 & 340 & 1464 \\
\hline 520 & 1345 & 520 & 1546 & 360 & 1537 & 360 & 1547 \\
\hline 550 & 1419 & 550 & 1631 & 380 & 1620 & 380 & 1629 \\
\hline 580 & 1493 & 580 & 1716 & 400 & 1702 & 400 & 1712 \\
\hline 610 & 1567 & 610 & 1800 & 420 & 1783 & 420 & 1794 \\
\hline 640 & 1639 & 640 & 1883 & 440 & 1865 & 440 & 1875 \\
\hline 670 & 1714 & 670 & 1967 & 460 & 1946 & 460 & 1957 \\
\hline 700 & 1786 & 700 & 2050 & 480 & 2026 & 480 & 2038 \\
\hline 730 & 1859 & 730 & 2132 & 500 & 2107 & 500 & 2087 \\
\hline 760 & 1931 & 760 & 2214 & 520 & 2187 & 520 & 2198 \\
\hline 790 & 2003 & 790 & 2296 & 540 & 2267 & 540 & 2278 \\
\hline 820 & 2074 & 820 & 2377 & 560 & 2345 & 560 & 2358 \\
\hline 850 & 2146 & 850 & 2459 & 580 & 2425 & 580 & 2437 \\
\hline 880 & 221 & 880 & 2538 & 600 & 2504 & 600 & 2516 \\
\hline 910 & & & & 620 & 2583 & 620 & 2595 \\
\hline 940 & & & & & & & \\
\hline
\end{tabular}

(a) TIME = elapsed time in days

(b) $\mathrm{BU}=$ burnup in $\mathrm{GJ} / \mathrm{kgU}$ 
TABLE 9. Elapsed Time and Burnup, $35 \mathrm{~kW} / \mathrm{m}$ Peak Power

\begin{tabular}{|c|c|c|c|c|c|c|c|}
\hline \multicolumn{2}{|c|}{ BWR $7 \times 7$} & \multicolumn{2}{|c|}{ BUR $8 \times 8$} & \multicolumn{2}{|c|}{ PWR $15 \times 15$} & \multicolumn{2}{|c|}{ PWR $17 \times 17$} \\
\hline $\operatorname{IIME}^{(\mathrm{a})}$ & $\mathrm{BU}^{(\mathrm{b})}$ & TIME & BU & TIME & 80 & TIME & $\mathrm{BU}$ \\
\hline & 2 & & 3 & 1 & $a$ & 1 & 5 \\
\hline$\frac{1}{5}$ & 13 & $\begin{array}{l}1 \\
5\end{array}$ & 18 & $\begin{array}{l}1 \\
5\end{array}$ & $\begin{array}{r}4 \\
23\end{array}$ & 10 & 61 \\
\hline 10 & 27 & 10 & 37 & 10 & 47 & 20 & 123 \\
\hline 20 & 55 & 20 & 74 & 20 & 96 & 40 & 247 \\
\hline 40 & 110 & 40 & 149 & 40 & 192 & 60 & 371 \\
\hline 70 & 192 & 70 & 260 & 60 & 287 & 80 & 493 \\
\hline 100 & 274 & 100 & 371 & 80 & 383 & 100 & 615 \\
\hline 130 & 356 & 130 & 481 & 100 & 478 & 120 & 736 \\
\hline 160 & 437 & 160 & 590 & 120 & 572 & 140 & 856 \\
\hline 190 & 518 & 190 & 698 & 140 & 666 & 160 & 975 \\
\hline 220 & 598 & 220 & 806 & 160 & 760 & 180 & 1094 \\
\hline 250 & 678 & 250 & 913 & 180 & 853 & 200 & 1212 \\
\hline 280 & 758 & 280 & 1020 & 200 & 946 & 220 & 1330 \\
\hline 310 & 837 & 310 & 1126 & 220 & 1039 & 240 & 1447 \\
\hline 340 & 916 & 340 & 1231 & 240 & 1131 & 260 & 1563 \\
\hline 370 & 995 & 370 & 1335 & 260 & 1223 & 280 & 1678 \\
\hline 400 & 1073 & 400 & 1439 & 280 & 1314 & 300 & 1793 \\
\hline 430 & 1151 & 430 & 1542 & 300 & 1405 & 320 & 1907 \\
\hline 460 & 1229 & 460 & 1645 & 320 & 1496 & 340 & 2021 \\
\hline 490 & 1306 & 490 & 1747 & 340 & 1586 & 360 & 2133 \\
\hline 520 & 1382 & 520 & 1848 & 360 & 1676 & 380 & 2246 \\
\hline 550 & 1459 & 550 & 1949 & 380 & 1765 & 400 & 2357 \\
\hline 580 & 1535 & 580 & 2049 & 400 & 1854 & 420 & 2468 \\
\hline $\begin{array}{l}610 \\
640\end{array}$ & 1611 & $\begin{array}{l}610 \\
640\end{array}$ & $\begin{array}{l}2149 \\
2247\end{array}$ & $\begin{array}{l}420 \\
440\end{array}$ & 1943 & & \\
\hline 670 & 1761 & 670 & 2346 & 460 & 2119 & & \\
\hline 700 & 1836 & 700 & 2443 & 480 & 2207 & & \\
\hline 730 & 1910 & 730 & 2540 & 500 & 2294 & & \\
\hline $\begin{array}{l}760 \\
790\end{array}$ & 1985 & & & 520 & 2381 & & \\
\hline $\begin{array}{l}190 \\
820\end{array}$ & $\begin{array}{l}2063 \\
2132\end{array}$ & & & $\begin{array}{l}540 \\
560\end{array}$ & $\begin{array}{l}246 / \\
2553\end{array}$ & & \\
\hline 850 & 2205 & & & & & & \\
\hline 880 & 2277 & & & & & & \\
\hline & & & & & & & \\
\hline 970 & $\begin{array}{l}2422 \\
2493\end{array}$ & & & & & & \\
\hline 1000 & 2565 & & & & & & \\
\hline
\end{tabular}

(a) TIME = elapsed time in days

(b) $\mathrm{BU}=$ burnup in $\mathrm{GJ} / \mathrm{kgU}$ 
where $B=\exp \left(-4+B U^{1 / 4}\right)$ and GAP is the initial (cold) diametral gap. It can be seen that once full power is reached ${ }^{(a)}$ this relationship is dependent only on burnup and, as shown in Table 10, reaches a maximum value after a burnup of $400 \mathrm{GJ} / \mathrm{kgU}$. This value corresponds to the time in the irradiation when the rapid temperature drop is completed.

After $400 \mathrm{GJ} / \mathrm{kgU}$, the temperature behavior is dependent upon the slowly decreasing linear heat rate (which will drop temperatures) and the increasing amount of fission gas present in the fuel-cladding gap (which will raise temperatures). At this point, fuel rod design begins to show a significant effect.

Although Figures 2-12 present the predicted fuel centerline and surface temperatures as a function of burnup, there is a better indicator of the

TABLE 10. GT3 Predicted Relocation as a Function of Burnup

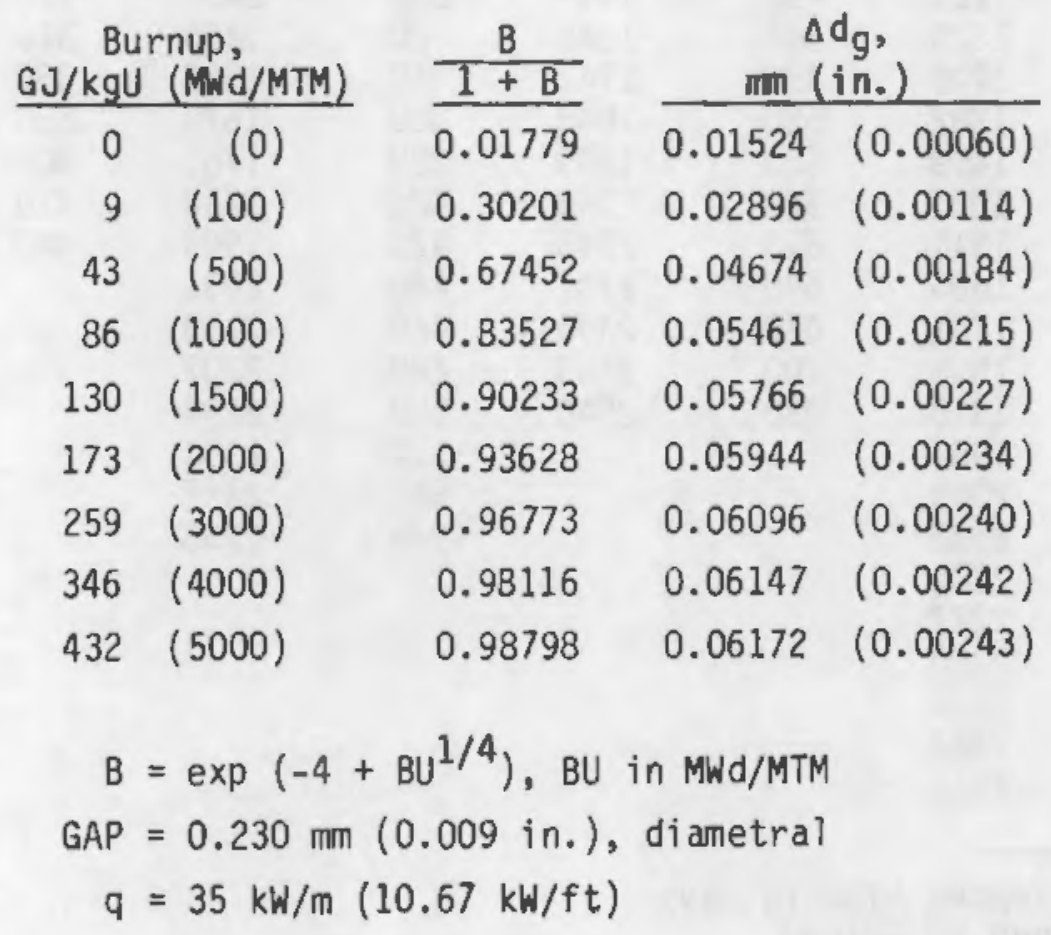

(a) The model as written assumes that once the fuel has relocated toward the cladding it cannot retreat; therefore, should the power drop, fuel relocation would not be reduced. 
changes within a fuel rod. Figures 13 and 14 present the thermal resistance ${ }^{(a)}$ of the fuel rods as a function of burnup. Appendix B provides a more detailed discussion of the resistance concept.

The BOL drop in fuel centerline and surface temperatures seen in Figures 2-12 is aiso observed in Figures 13 and 14; however, after this temperature drop differences between the rod designs are observed. The BWR centerline temperatures in Figures 2-4 and 7-9 appear fairly constant with only a slight increase at EOL. However, the resistance for the 1-atm BWR rods begins increasing immediately after the fuel relocation is completed. This increase in resistance is directly attributable to the release of fission gas to the fuel-cladding gap, which degrades the gap conductance.

This emphasizes the importance of increasing the initial amount of helium in a fuel rod. Notice the resistance for the 3-atm BWR rod: Initially it is constant (after relocation is completed) and does not begin to rise until later in life. This is because fission gas release cannot degrade the thermal conductivity of the fill gas as quickly and thus reduce the gap conductance.

The PWR centerline temperatures in Figures 5, 6, 10, and 11 are seen to decrease with burnup. Figures 13 and 14 show that the resistance $f$ or these rods remains constant during the assumed power history. Therefore, the observed temperature drop is directly attributable to the power decrease during the power history. The constant resistance indicates that fission gas release has a negligible effect on the gap conductance. As with the 1-atm BWR rods, resistance for the 1-atm PWR rod begins increasing after relocation is complete (Figure 14). At EOL, its resistance is $30 \%$ higher than that of the pressurized PWR rods.

Figure 14 indicates that for approximately equal power and after relocation has been assumed, the $7 \times 7$ BWR rod has a lower resistance than the $8 \times 8$ BWR rod and the $15 \times 15$ PWR rod has a lower resistance than the $17 \times 17$ PWR rod. It was noted earlier that at BOL increasing gap sizes lead to higher fuel temperatures, which is the opposite of the effect here. After relocation each rod

(a) The thermal resistance is defined as the difference between the fuel centerline temperature and coolant temperature divided by the local linear heat rate. 


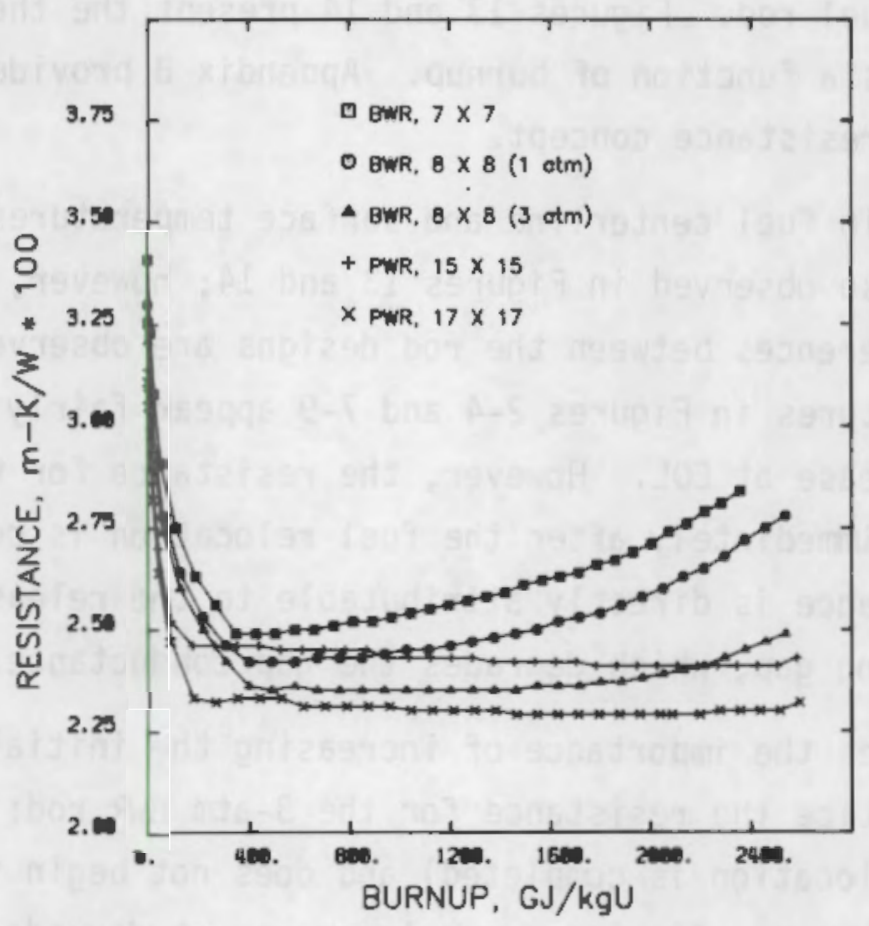

FIGURE 13. Thermal Resistance History for Case 1

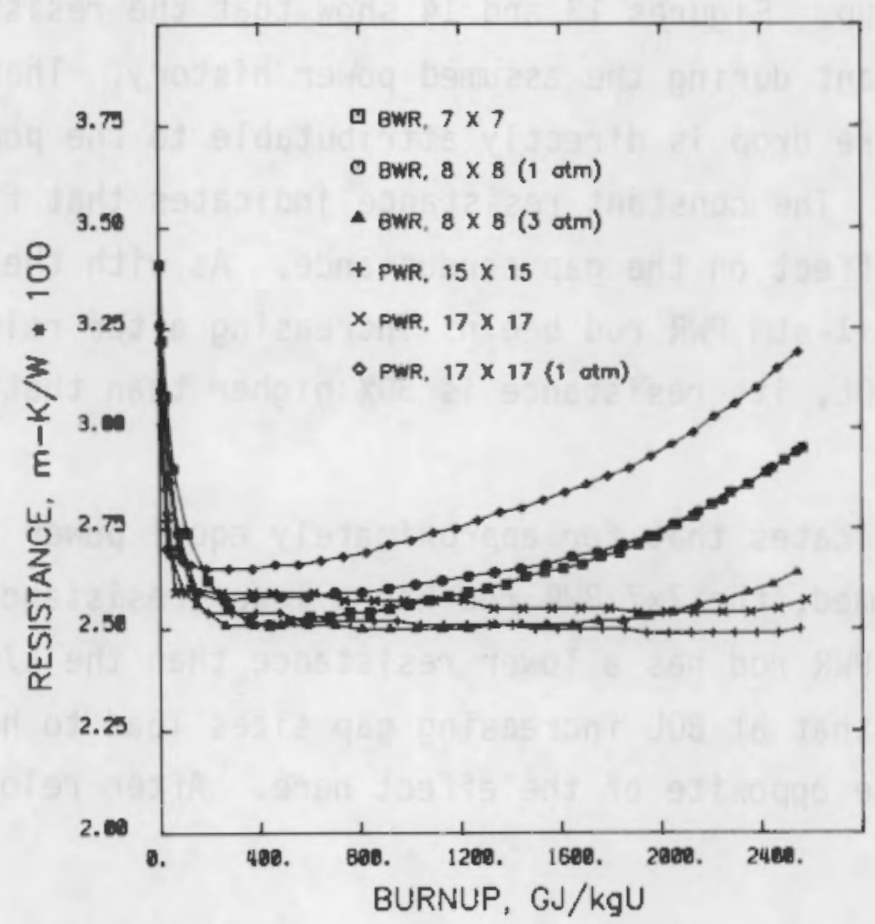

FIGURE 14. Thermal Resistance History for Case 2 
type has approximately the same gap conductance and fuel surface temperature, which would seem to lead to equal resistances. However, to produce the same linear heat rod, the smaller diameter rod must have a higher power density. This then results in a higher centerline temperature and the observed higher resistance for the smaller diameter fuel rods.

\section{TEMPERATURE UNCERTAINTY}

Figures 2-12 present the estimated uncertainty for temperature and stored energy as upper and lower bounds. Several general observations can be made from these plots.

- The uncertainty for fuel surface temperature decreases when fuelcladding contact occurs because it is assumed that gap conductance is better defined under contact conditions.

- The width of the uncertainty band for fuel surface temperature increases with burnup because of an increasing contribution from the fuel relocation factor $\left(F_{p r}\right)$.

- Although the fuel surface temperature uncertainty decreases under fuel-cladding contact conditions, the uncertainty of centerline temperature and stored energy does not decrease at this point. This occurs because power is the principal driver of fuel temperatures and is therefore the major contributor to and dominator of fuel uncertainties. If the assigned power uncertainty was less than $15 \%$ (30), a decrease in fuel centerline temperature and stored energy uncertainty would be seen when the fuel surface temperature uncertainty decreases. This result is also seen with Monte Carlo analysis.

While the temperature and absolute uncertainty response differs from rod to rod, the relative uncertainty (expressed as a percent of the predicted parameter) is nearly equal between rod designs. This particularly holds true at $\mathrm{BOL}$ before burnup effects make substantial changes to the operating conditions. Therefore, parametric plots have been developed that present the relative uncertainty for centerline temperature and stored energy as a function of power uncertainty. 
Figures 15-17 present BOL relative uncertainty plots for power levels of 20,35 , and $50 \mathrm{~kW} / \mathrm{m}$; and Table 11 lists basic assumptions. Three assumptions for fuel thermal conductivity, specific heat, and flux depression uncertainty are used to provide a band of relative uncertainty. It can be noted that

- Uncertainties for fuel centerline temperature and stored energy are nearly linear with power uncertainty when power uncertainty exceeds $20 \%$.

- Uncertainties for fuel thermal properties and radial power distribution define the minimum uncertainty for temperature, stored energy, and the width of the uncertainty band as a function of power.

Table 12 presents the uncertainties used for developing parametric plots for EOL conditions. Because of the difference in time-dependent response between BWR and PWR designs, a single plot cannot be developed for both designs. Results for a BWR rod are shown in Figures 18-20 and results for a PWR rod are shown in Figures 21-23. The same general trends observed in the BOL figures can be seen with the major exception being the lower values of relative uncertainty (relative uncertainty is inversely proportional to temperature). Although the higher EOL temperatures have a lower relative uncertainty, the absolute uncertainty (in $\mathrm{K}$ for temperature or $\mathrm{J} / \mathrm{m}$ for stored energy) is larger than at BOL because of the higher temperatures at EOL.

The uncertainty estimates provided in this section serve a dual purpose. First, as presented, they provide confidence bands for modeling predictions of fuel rod thermal behavior; i.e., a prediction of $X$ is good within $\pm Y \%$. Secondly, because the models are predicting behavior, it would be expected that observed data would fall within the band described by the uncertainty anaiysis if the modeling is done correctly. This leads into the next subject: verification and validation of models and computer implementations of those models. 


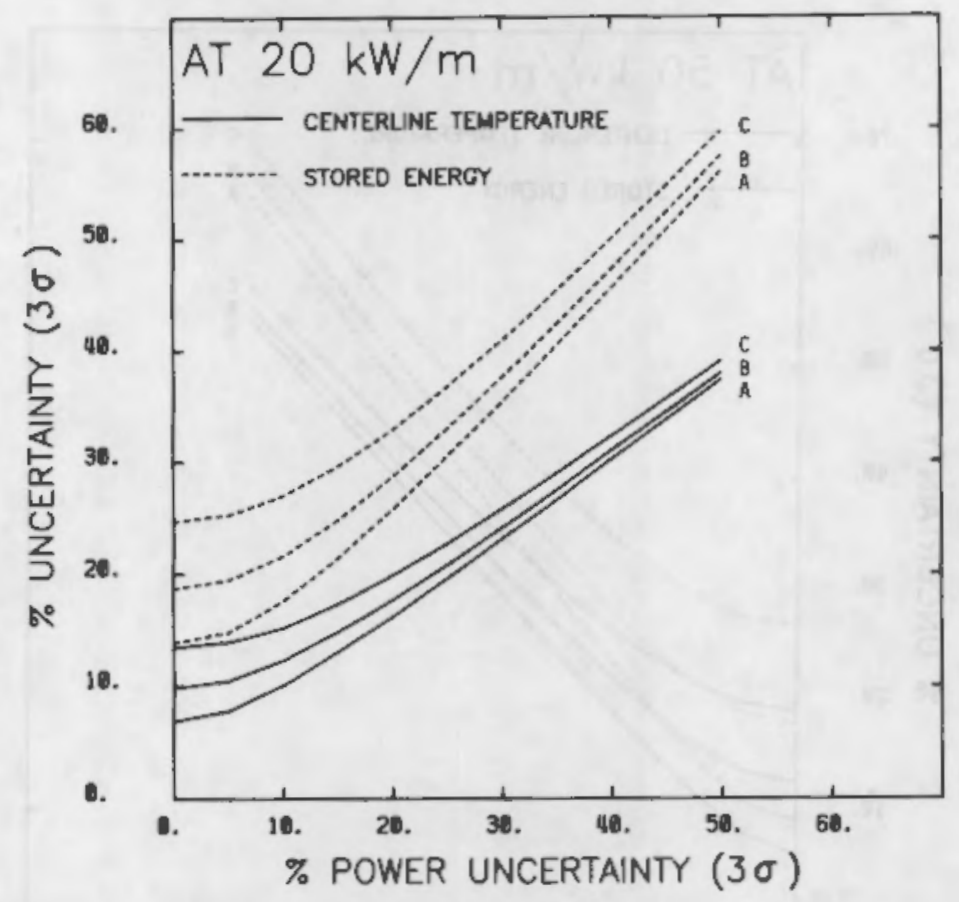

FIGURE 15. Beginning-of-Life Relative Uncertainty for a LWR Rod at $20 \mathrm{~kW} / \mathrm{m}$

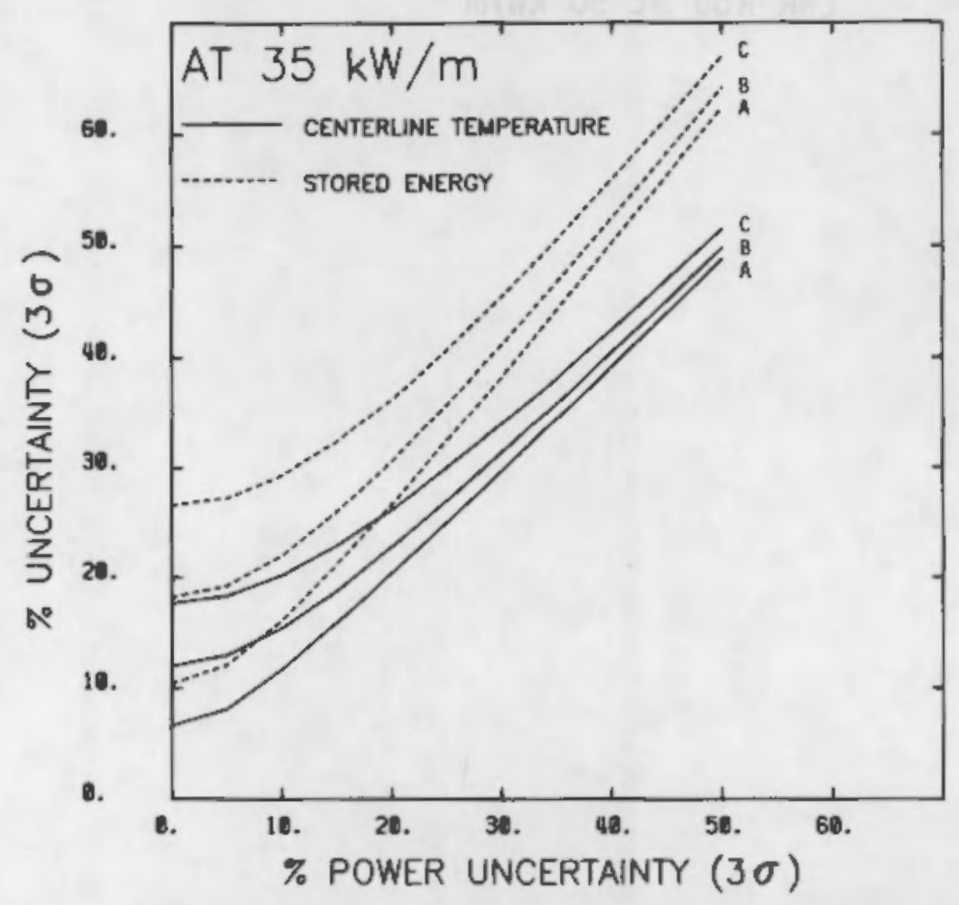

FIGURE 16. Beginning-of-Life Relative Uncertainty for a LWR Rod at $35 \mathrm{~kW} / \mathrm{m}$ 


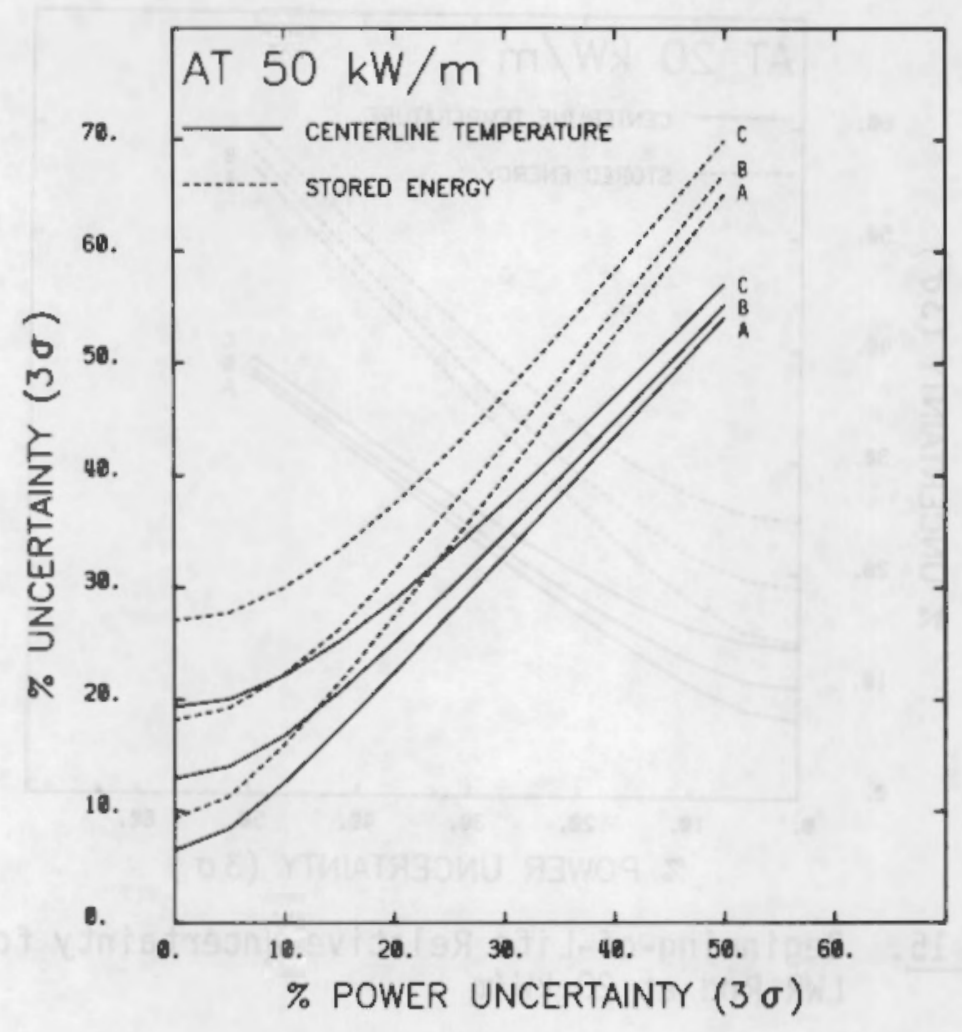

FIGURE 17. Beginning-of-Life Relative Uncertainty for an LWR Rod at $50 \mathrm{~kW} / \mathrm{m}$ 
TABLE 11. Basic Assumptions for BOL Uncertainty Parametric Study THERMAL RESPONSE

\begin{tabular}{|c|c|c|c|c|c|c|}
\hline \multirow{2}{*}{$\begin{array}{l}\text { Power, } \\
\mathrm{kW} / \mathrm{m}\end{array}$} & \multicolumn{2}{|c|}{$\begin{array}{l}\text { Resist ance, (a) } \\
\mathrm{mK} / \mathrm{W} \cdot 100\end{array}$} & \multicolumn{2}{|c|}{$\begin{array}{l}\text { Centerline } \\
\text { Temperature, } \mathrm{K}\end{array}$} & \multicolumn{2}{|c|}{$\begin{array}{l}\text { Stored Energy, (b) } \\
\mathrm{J} / \mathrm{m}\end{array}$} \\
\hline & & PAIR & & & Bin & $P L R$ \\
\hline 20 & 2.6 & 2.6 & 1073 & 1096 & 94,400 & 55,900 \\
\hline 35 & 2.6 & 2.6 & 1463 & 1479 & 154,900 & 90,500 \\
\hline 50 & 2.9 & 2.9 & 1980 & 2021 & 240,600 & 145,200 \\
\hline
\end{tabular}

INPUT UNCERTAINTY

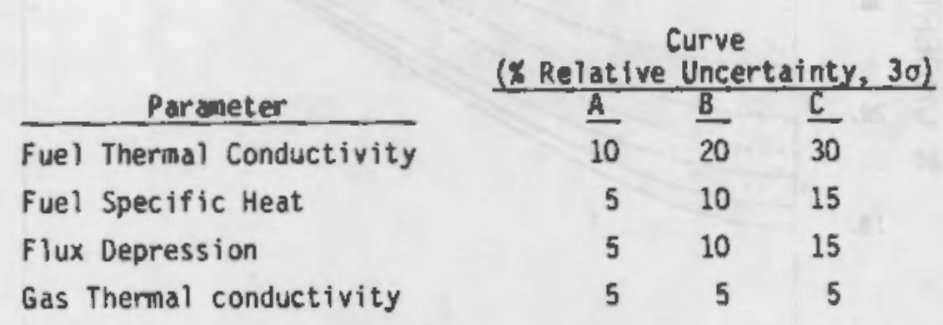

(a) Resistance based on GT3 predictions with fuel relocation. Higher resistance at $50 \mathrm{~kW} / \mathrm{m}$ is due to reduced fuel thermal conductivity at higher temperatures.

(b) Stored energy is relative to coolant temperature.

TABLE 12. Basic Assumptions for EOL Uncertainty Parametric Study THERMAL RESPONSE

\begin{tabular}{|c|c|c|c|c|c|c|}
\hline \multirow{2}{*}{$\begin{array}{l}\begin{array}{l}\text { Power, } \\
\mathrm{kH} / \mathrm{m}\end{array} \\
\end{array}$} & \multicolumn{2}{|c|}{$\begin{array}{l}\text { Resistance, (a) } \\
\text { MK/W } 100\end{array}$} & \multicolumn{2}{|c|}{$\begin{array}{c}\text { Centerline } \\
\text { Temperature, } K\end{array}$} & \multicolumn{2}{|c|}{$\begin{array}{l}\text { Stored Energy, (b) } \\
\mathrm{J} / \mathrm{m}\end{array}$} \\
\hline & & & & & BWR & PMR \\
\hline 20 & 4.2 & 3.1 & 1393 & 1198 & 179,600 & 71,200 \\
\hline 35 & 3.9 & 3.0 & 1917 & 1629 & 270,100 & 112,000 \\
\hline 50 & 3.7 & 2.9 & 2393 & 2052 & 349,300 & 149,500 \\
\hline
\end{tabular}

INPUT UNCERTAINTY

\section{Parameter}

Fuel Thermal Conductivity

Fuel Specific Heat

Flux Depression

Gas Thermal conductivity

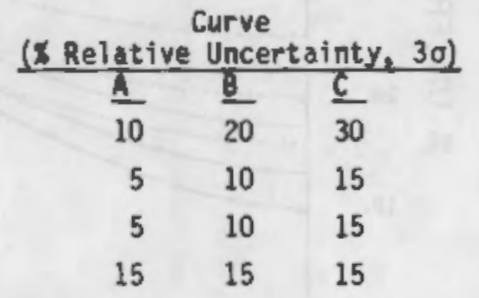

(a) Because of fission gas release, resistance is gap dominated.

(b) Stored energy is relative to coolant temperature. 


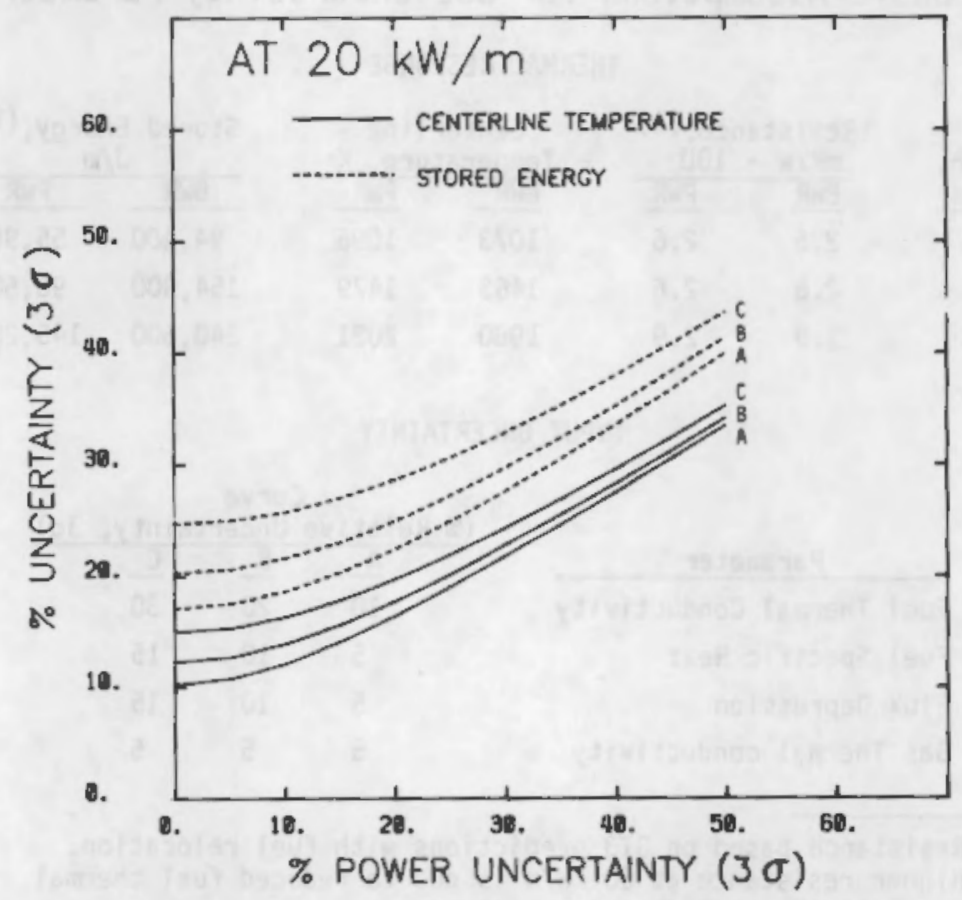

FIGURE 18. End-of-Life Relative Uncertainty for a BWR Rod at $20 \mathrm{~kW} / \mathrm{m}$

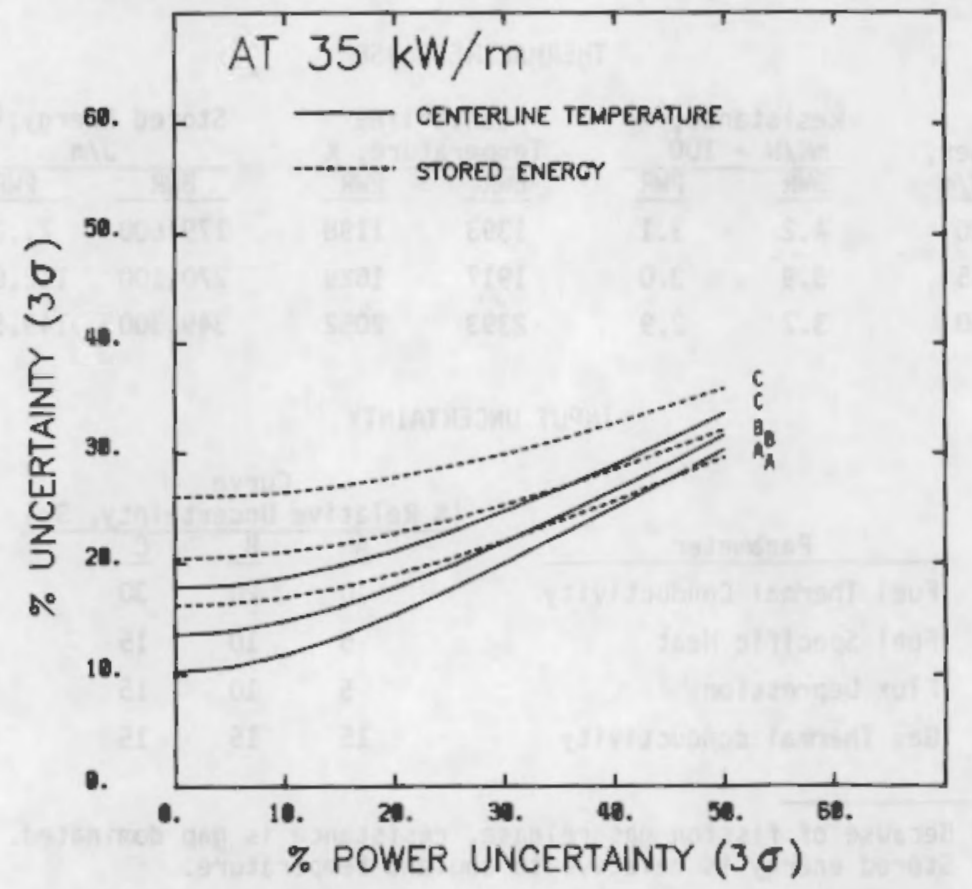

FIGURE 19. End-of-Life Relative Uncertainty for a BWR Rod at $35 \mathrm{~kW} / \mathrm{m}$ 


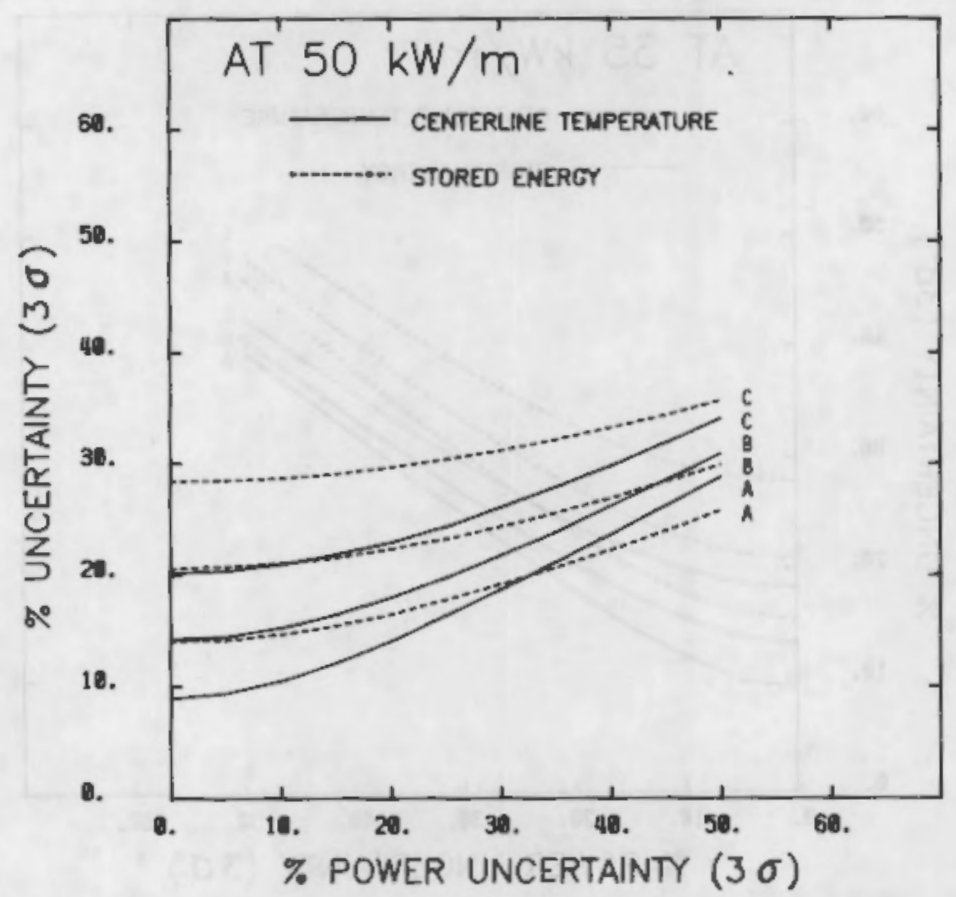

FIGURE 20. End-of-Life Relative Uncertainty for a BWR Rod at $50 \mathrm{~kW} / \mathrm{m}$

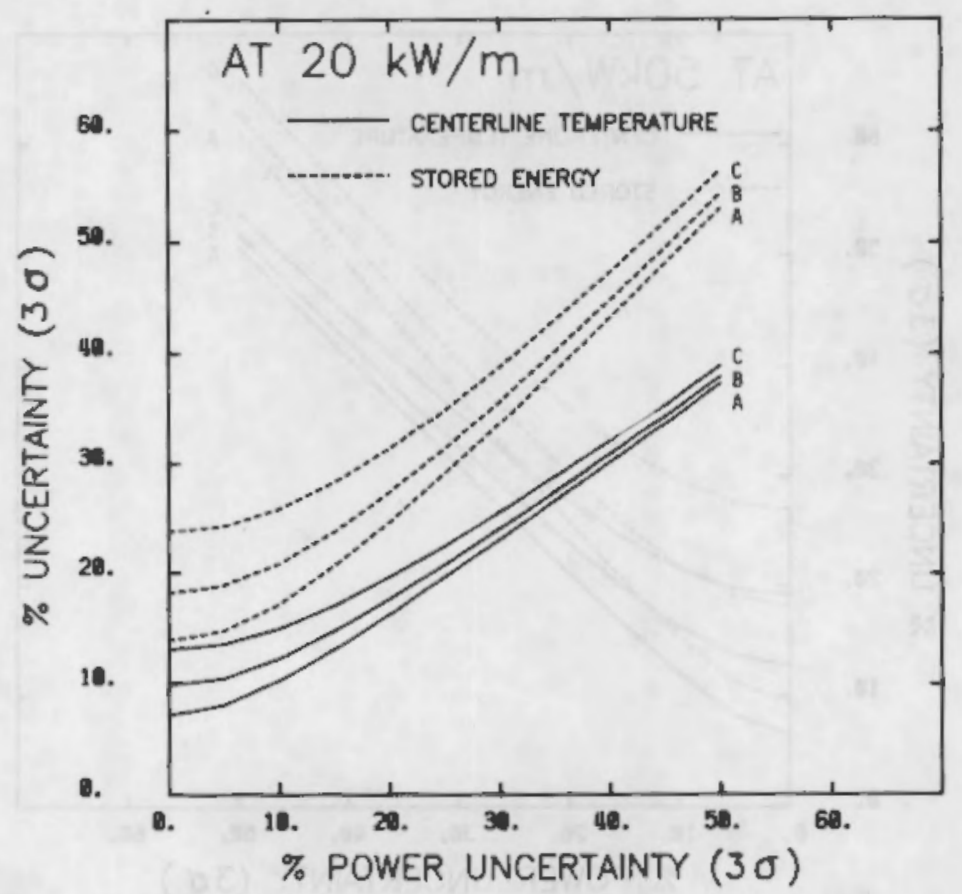

FIGURE 21. End-of-Life Relative Uncertainty for a PWR Rod at $20 \mathrm{~kW} / \mathrm{m}$ 


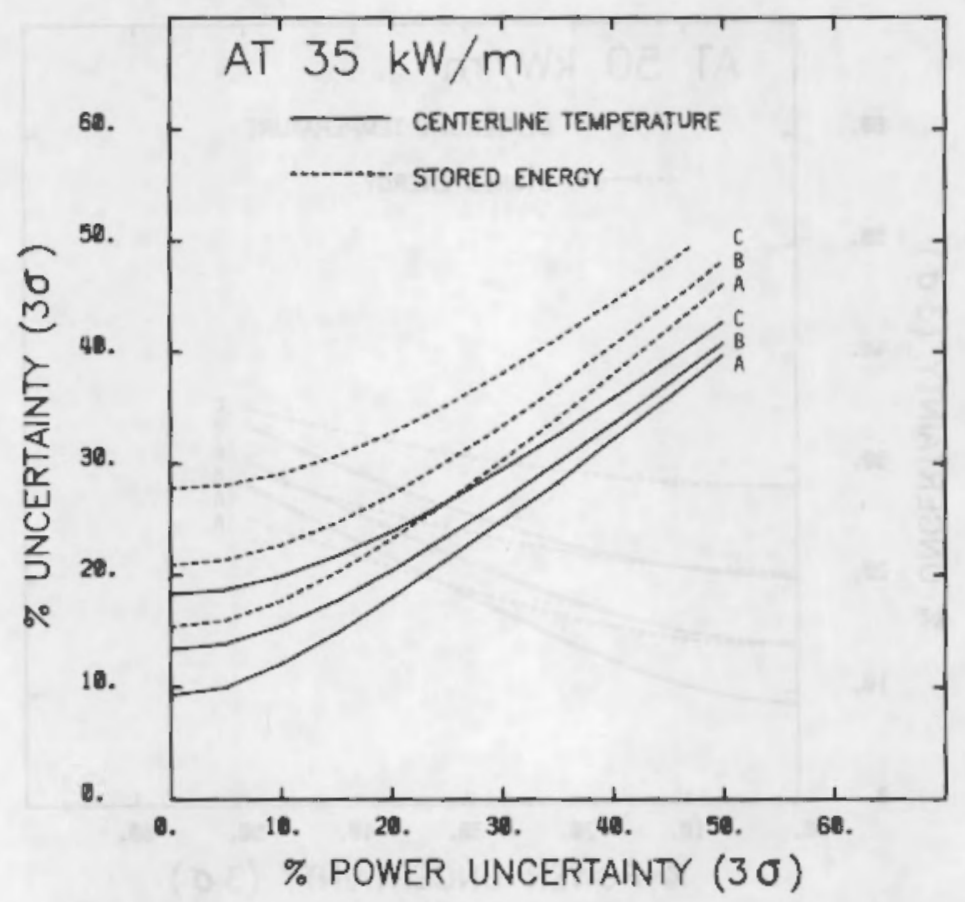

FIGURE 22. End-of-Life Relative Uncertainty for a PWR Rod at $35 \mathrm{~kW} / \mathrm{m}$

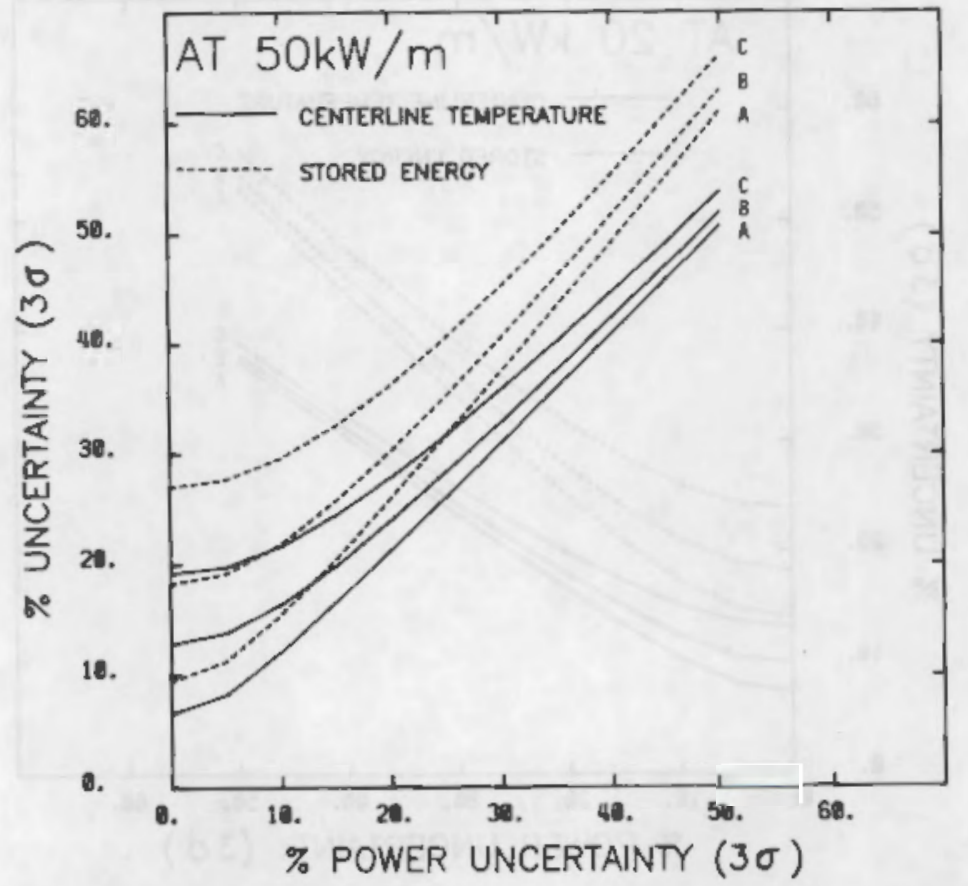

FIGURE 23. End-of-Life Relative Uncertainty for a PWR Rod at $50 \mathrm{~kW} / \mathrm{m}$ 


\section{MODEL AND CODE VERIFICATION}

The end objective of developing fuel rod behavior models is to be able to predict how fuel rods will behave. Once the desired models have been selected/ developed, implemented as a computer code, and a prediction obtained, the question arises: How good is the prediction? Points to be considered when answering this question include accuracy of the chosen models, quality of the input to the computer code, and, in general, confidence in the code.

This section presents a qualitative discussion of the modeling process and code validation. Examples of several techniques that have been used to support the validity of computer codes have been included.

\section{MODELING AND REALITY}

The process of model development is usually not discussed when model validation is the main interest; however, this process is a factor in assessing the confidence to be given to a model. It also provides a common ground from which the terms model verification, validation, and evaluation may be later defined.

Mar (1974) in discussing the process of structuring models states that the development follows a general pattern of explicit mental models, correlative models, and casual models. First, a model is constructed based on experience, intuition, and limited data to provide quantitative insights. Second, as additional quantitative data become available, a model is developed based on those data and empirical correlation of significant parameters. Third, physical theories plus quantitative data are employed to develop a model. In the physical sciences, theories and data are usually available to develop basic models that are generally accepted. However, even in well-developed physical science areas (for example, nuclear fuel thermal and mechanical performance) detailed theory and extensive data may not always be available to describe a model completely. Some components of a model will therefore be based on empirical correlations and others only on intuition, experience, and limited data. Recognition of these stages or steps in model development is important when confidence in a model is assessed. 
The basic components inherent in the modeling process are the true physical situation, the observed physical situation, accepted scientific knowledge, and the resulting theoretical model. The relationship among these components is shown in Figure 24. It is the objective of the modeler to develop a theoretical model that adequately depicts the true physical situation. The theoretical model is based on accepted scientific knowledge and the observed or perceived physical situation.

The actual physical situation may be described as the true state of nature. For nuclear fuel performance this includes the actual state of the fuel, cladding, fill gas, coolant, and many additional factors associated with nuclear reactor operation. The factors may be classified roughly into initial conditions, controllable operating conditions, and resulting operating conditions or states. Initial conditions are typified by fuel, cladding, and general reactor characteristics. Controllable operating characteristics are power level and coolant conditions. Operating characteristics are those that are determined by initial conditions and controllable operating characteristics. These include fuel radial temperature profile, cladding temperature, fission gas composition, and fuel-cladding mechanical behavior as well as many others.

The process of observing or perceiving the true physical situation induces noise or error in the resultant observations or perceptions. This arises from the process of measuring a certain characteristic or when perceptions are filtered by previous knowledge. In many cases the error introduced is inconsequential to the development of the theoretical model; in others it may actually lead to an incorrect model or at least an incorrect empirical parametric formulation. For example, if a thermocouple is placed in the center of the fuel to measure the fuel centerline temperature, it may actually change the physical

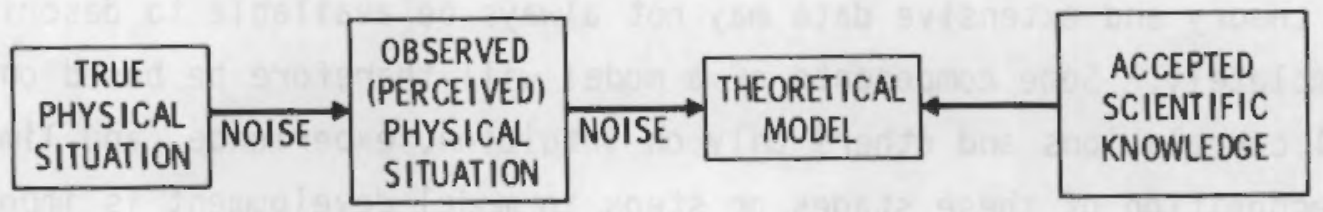

FIGURE 24. Components of Modeling Process 
situation: Since part of the fuel is removed to accomplish the measurement, the centerline temperature is changed. Although adjustments in the measured centerline temperature can be made, it is the resulting adjusted temperature that enters into the development of the theoretical model.

An advantage of developing a theoretical model in the physical sciences is the rich history of theory and related data available; hence, much of the model development is based on accepted scientific knowledge. However, the use of scientific knowledge in model development is not always done without the introduction of noise (error). For example, in constructing a theoretical model to describe fuel rod behavior, it is often necessary to approximate or simplify certain aspects of accepted scientific knowledge (represented in Figure 24 as the introduction of noise between components). In GT3 the radial temperature profiles can be theoretically described by a coupled set of equations in three dimensions. In actual application the equations are simplified to include only the radial direction. This approximation, and the numerical algorithm used for its solution, introduces noise (for lack of a better term) to the information.

The observed or perceived physical situation is also a part of the development of a theoretical model. In many cases there is no accepted theory; only empirical information is available for formulating possible mechanisms. Much of this information is gained from observing and analyzing previous experiments. In some cases a theoretical mechanism can be formulated but an empirically determined parametric function must be used to implement the mechanism as part of a working model. In this case the theoretical model is based on actual physical situations, but approximation and/or noise is introduced by the process of observing the actual situation and by simplifying the observed situation by means of a mathematical formulation.

A distinction is made between the theoretical model, which includes the mathematical fomulation and supporting empirical parameterizations, and the implementation of the theoretical model as a computer code or model. Confidence in the computer model is enhanced by increased understanding of the basis for the theoretical model. This understanding may be increased by providing 
in separate documentation a detailed description of the philosophy used in developing the model, the reasoning behind the selection of the approach, and the components of the model.

It is necessary to emphasize that documenting the development process for a theoretical model is an important part in the subsequent assessment of the model as a computer code. The documentation of the process, including the model selected and the reasons for the choice, is the mechanism for informing users. The documentation must give a clear definition of the system modeled and $i$ ts proposed use plus a clear understanding of the status of theory, experience, intuition, and data for each component in the system. This does not imply that each component of the model must be state of the art, only that its relationship to the state of the art be given.

A computer code is the implementation of a theoretical model, and it is a distinct step in the modeling process and should not be cambined with the development of the theoretical model. It is recognized that interactions occur between the steps and iterations do occur in the process. The construction of the computer code is not a direct translation fram a detailed theoretical model.

Implementation of the theoretical model usually requires iterative solutions and/or numerical approximations. For example, in GT3 and the FRAPCON code series, the theoretical model for radial temperature profile is solved by using the technique of orthogonal collocation of weighted residuals. To develop confidence in these codes, it is not only necessary that the theoretical model be accepted but also the choice of the numerical technique. Documentation supporting the technique in the present application is necessary for developing confidence in the model.

Computer code implementation may involve additional approximations or assumptions. As previously indicated, numerical approximations are usually required to implement the theoretical model. Their use introduces noise between the computer code implementation and the theoretical model. The implementation introduces coding errors or inadequacies. Hopefully, these can be easily eliminated. The finite accuracy of the computer can also introduce 
noise in implementing the theoretical model. All of the these factors must be considered.

The theoretical model and its computer code implementation can be distinguished by the code's dependence on a particular computer operating system; the theoretical model is independent of computers. Thus, the model must stand alone and be evaluated accordingly. Justification and documentation of the model must be sufficient to allow an independent assessment of its merits. This is especially critical when the model is to be used in situations where it is impossible to check model predictions against actual observed data. The computer code cannot be assessed independently of its computing system environment; therefore, a change in the computing system requires a reevaluation of and occasionally a redesign of the code even though the theoretical model has not changed.

\section{COOE VERIFICATION}

Code verification is defined as the process of verifying that the computer code implementation of the model is done correctly. Commonly, code verification is assumed to be the same as debugging a computer code; however, debugging is only a part of code verification. When numerical approximations have been used, the solution accuracy needs to be evaluated and checked for the possibility of multiple solutions. The stability of algorithms must be investigated, especially for models predicting a time history. Theoretically an algorithm may be known to be stable but the finite accuracy of the computer may invalidate the stability.

Code verification encompasses only the theoretical model, the code implementation, past observed physical situations used to develop the model, and elements of accepted scientific knowledge (Figure 25). The only concern is: Does the computer code in the present computing environment accurately represent the theoretical model? At this stage it is not of concern whether the computer code agrees with the actual or observed physical situation.

In addition to careful documentation of the design and implementation of the computer code and comparison of code predictions with analytical results 


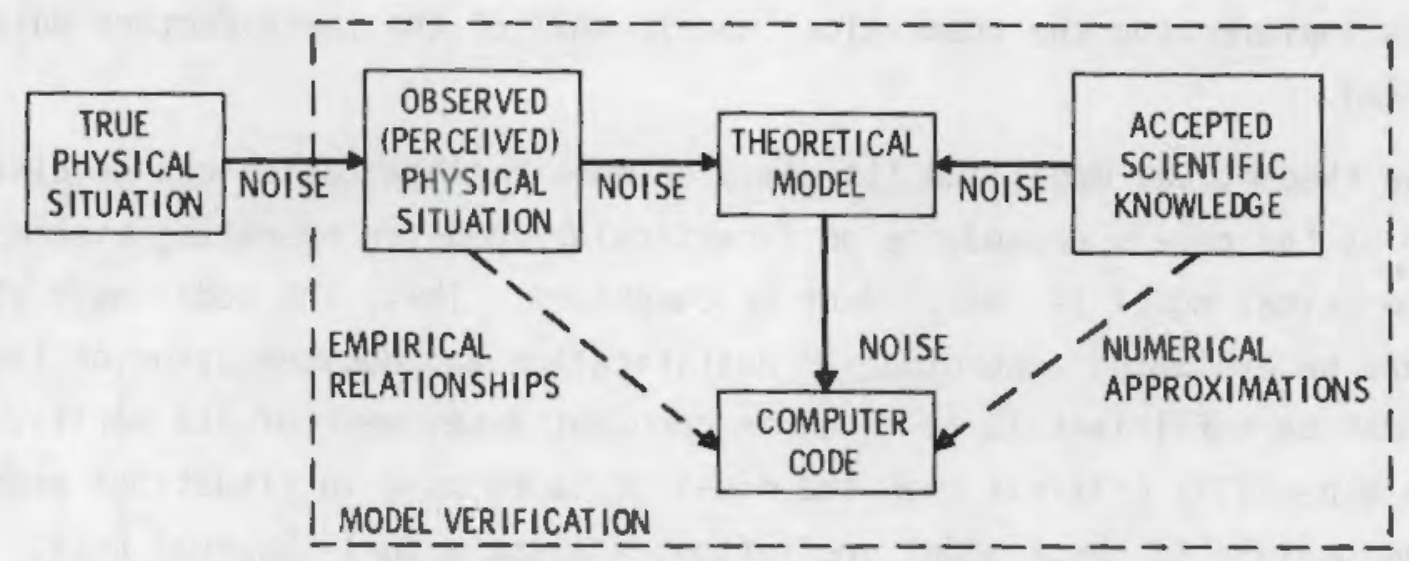

FIGURE 25. Computer Code Implementation of Theoretical Model

where possible, active debugging is important. Active debugging concerns the process of running the code to find hidden errors, which is not a trivial task for complex codes such as GT3. The number of input variables, their allowable ranges, and the number of options quickly result in a large number of combinations to be checked in separate computer runs. Statistical concepts of experimental design can be used to reduce the number of computer runs required. One application of this concept is given by Mckay, Conover, and Beckman (1979), although in a different context.

Except with relatively simple models, a computer code is rarely completely verified. Verification is limited by the knowledge available about what the theoretical model implies in specific cases. This is especially true concerning magnitudes of output variables. Generally, the only comparison available is whether the result "looks reasonable." Another limitation is the inability to try all possible combinations. Stated in a statistical context, an inference is required in stating that a computer code is verified. The strength of or confidence placed in this inference depends on all aspects of model verification and not just selected computer results.

\section{MOOEL ANALYSIS}

Model analysis is concerned with gaining an understanding of the theoretical model. The importance of selected variables or the change in an output 
variable as a function of changes in input variables may be investigated. Although model analysis can be completed on the theoretical model directly, it is usually conducted by using the computer code implementation in a sensitivity study. In general, the emphasis is on gaining an understanding of the relationship between output and input variables; and in some cases the contribution of complete submodels is investigated.

Model analysis can contribute to the development of confidence in a theoretical model through an increased understanding of the inner workings of the model. Secondary consequences of a theoretical model may also be checked through model analysis to further increase confidence in the model. However, model analysis as defined here will not directly support the correctness of the theoretical model in representing the actual physical situation.

\section{MODEL VALIDATION}

Model validation may mean many things. For present purposes a relatively restricted definition is given. Model validation is the comparison of output from a computer code implementation of a theoretical model to data obtained from an observed physical situation. Preferably the observed data are the product of a planned experiment so that the conditions under which the data are collected are well controlled and measured.

The term model validation has been criticized by many authors. Mitchell and Wilson (1979) have given some of the main reservations:

- It implies that it is possible to establish the validity of a model.

- It ignores the role of the use of the model.

- It ignores the temporary nature of model "validity."

- It ignores the role of subjectivity in the validation process. Although these reservations apply to a more general definition of model validation than presently assumed, they do highlight important points both in model validation and in developing confidence in a model. In fact, the concept of developing confidence in a model coincides with some authors' definition of model validation.

Since the use of the model must be considered as an integral part of model validation, it is generally not possible to validate a model for all possible 
uses. Therefore, the first step in model validation must be a clear definition of the use for which the model is being validated.

Model validation, as applied in the usual way, has a sense of concreteness associated with it. The association of the validation with an end use erodes that idea of finality or definiteness, and it is further eroded when the subjective and temporary nature of the validation is accepted. Validation is subjective since the physical situation is observed with error, which affects not only the comparison of the output with observed data but also the selec$t$ ion of input values for the code.

After clearly stating the end use, the next step is to determine how precisely and accurately the identified variables must be predicted for the model to be considered useful. This viewpoint stresses a yes/no decision on model validity for a particular use, and it presumes that it is possible to obtain observed data to make the necessary comparisons. If adequate data cannot be obtained, a formal determination of the validity of the model for that use cannot be made.

The term model validation implies that it is possible to establish the validity of the model. If a model has not yet been validated, the implication is that if more effort were expended (i.e., more experimental data gathered and compared to model output) it would only be a matter of time before the model would be validated. This implication ignores the dependence on the end use of the model and the known introduction of noise between the theoretical model, the true physical situation, and accepted scientific knowledge. The useful question is: Does the computer code implementation agree closely enough with the physical situation for a specific use? Segments of the model may be known to be invalid (incorrect or only approximations); but if the model output is satisfacory for the end use, the model remains useful. As a result the model may be inadequate for another use.

Conceptually, the process of model validation is simple. An end use of the model is described and criteria are selected for determining if the model is adequate enough for the use. An experiment is planned and executed or previously gathered data are found that meet the requirements for the 
validation exercise. Values for the input variables are chosen to match the experimental conditions and the computer model is executed. The model output is then compared to corresponding observed experimental data. In practice, however, there are many difficulties that may arise.

Since most models produce a time history on a number of variables, a large number of individual comparisons are definable. The comparisons that are to be included in the validation must be described clearly, for it is probable that not all of them will indicate that the model is valid. A subjective assessment, or in rare cases a quantitative measure, must be made concerning overall model validity. It is easy to envision a large number of comparisons for even a single physical situation.

In practice, determining the validity of a model for even a single end use will require that a number of physical situations be included in the validation process. Clearly, obtaining observed data will not be feasible for all situations; and, therefore, some representative cases must be selected. In the physical sciences the continuity or smoothness of most physical processes is a distinct advantage. The end use of the model naturally leads to a definition of ranges appropriate for each of the input variables to the model. More generally this is termed the feasible region for the input variables for that end use. By using the basic concepts of experimental design, a series of experiments may be defined that will allow an inference to be made on the validity of the model. This is a luxury that is available only if it is possible to conduct experiments with input variables in the feasible region. The concept of designed experiments can be profitably used in model verification where observations of the physical situation are not needed.

More commonly a few previously conducted experiments and additional observations are available from nomal operations and routine measurements. Rarely do these experiments and observations result in data covering the feasible region of input variables, and only occasionally are they near the feasible region. Clearly the formal validation process is curtailed or even invalidated in that particular case and must be replaced in this situation by a face validation. Face validation, which is much weaker than formal validation, is 
based on comparisons made outside the feasible region, if any; confidence in the model is developed through model verification, analysis, and documentation.

Given the input values to the code, the output is fixed and may differ from the observed data due to

- observation error in the data

- input to the code that differs from the true physical situation - an inadequate model.

Any or all of these can be present in a model validation exercise, and it is difficult to determine if there is an inadequacy in the model that affects the usefulness of the model when other causes for discrepancy are possible.

In most validation studies it is assumed that input variables (initial conditions) are known without error. All differences between model output and observed data are attributed either to model inadequacies or experimental error in the observed output variables. Typically in the physical sciences it is possible to estimate (guesstimate?) the error in the observed variables. The predicted output is compared to the observed data with its associated error limits. If the prediction is within the error limits, it is inferred that the model is adequate; if not, model adequacy is questioned. This procedure depends heavily on the correct assessment of the error associated with the observed data. Hence, model validation depends on an error analysis of the observed data or physical situation; when errors in input variables are admitted, the comparison becomes even more subjective.

An alternative approach to model validation is available that assumes that there are errors in input and output variables. Conceptually the idea is to adjust the model input variables so that model output agrees exactly with observed output data. The model validation comparison is then between observed input values and empirically determined model input values. The model is validated when the input values do not differ significantly. The process, however, is complicated by having to determine model input values; and a trial-and-error approach can be expensive. A further complication is the 
possibility that more than one choice of model input values can lead to the same model output values. Knowledge of such multiplicity can be very enlightening however.

The preceding comments certainly do not cover all the topics connected with developing confidence in a model nor are those included discussed in any depth. However, those individuals looking for a well-defined procedure that will lead to the validation of a model for all applications for which it was designed will be disappointed; those looking for specific statistical techniques to use have not been satisfied. These comments are not what model developers want to hear.

Briefly, the position taken is that while it is not possible to validate a model in the usual sense, it is possible to develop confidence in a model. The level of confidence will depend upon who is studying the model and will change with time. Model developers and users must make a distinction between face validity and formal validity. Formal validity is only possible when observed data for a feasible region of input variables are available for comparison with model output predictions; face validity depends only on model documentation, verification, and analysis and is therefore weaker. This does not imply that gaining face validity for a model is an easy task; in a sense, it is more difficult since it rests solely on communication of model characteristics and defense of the correctness of the logic behind the model.

\section{EXAMPLES OF VALIDATION}

Having discussed the process and problems of validating theoretical models, it is useful to present several examples of model validation.

The first example is the code verification and model validation of GT3 (Lanning, Panisko, and Mohr 1978). The work was divided into three basic areas

- Mathematical consistency of the coding was verified by comparison to hand calculations and analytical solutions. Simplifications in the code, such as elimination of temperature-dependent properties, were often necessary for this comparison. Figure 26 illustrates the verification of the steady-state temperature calculator. 


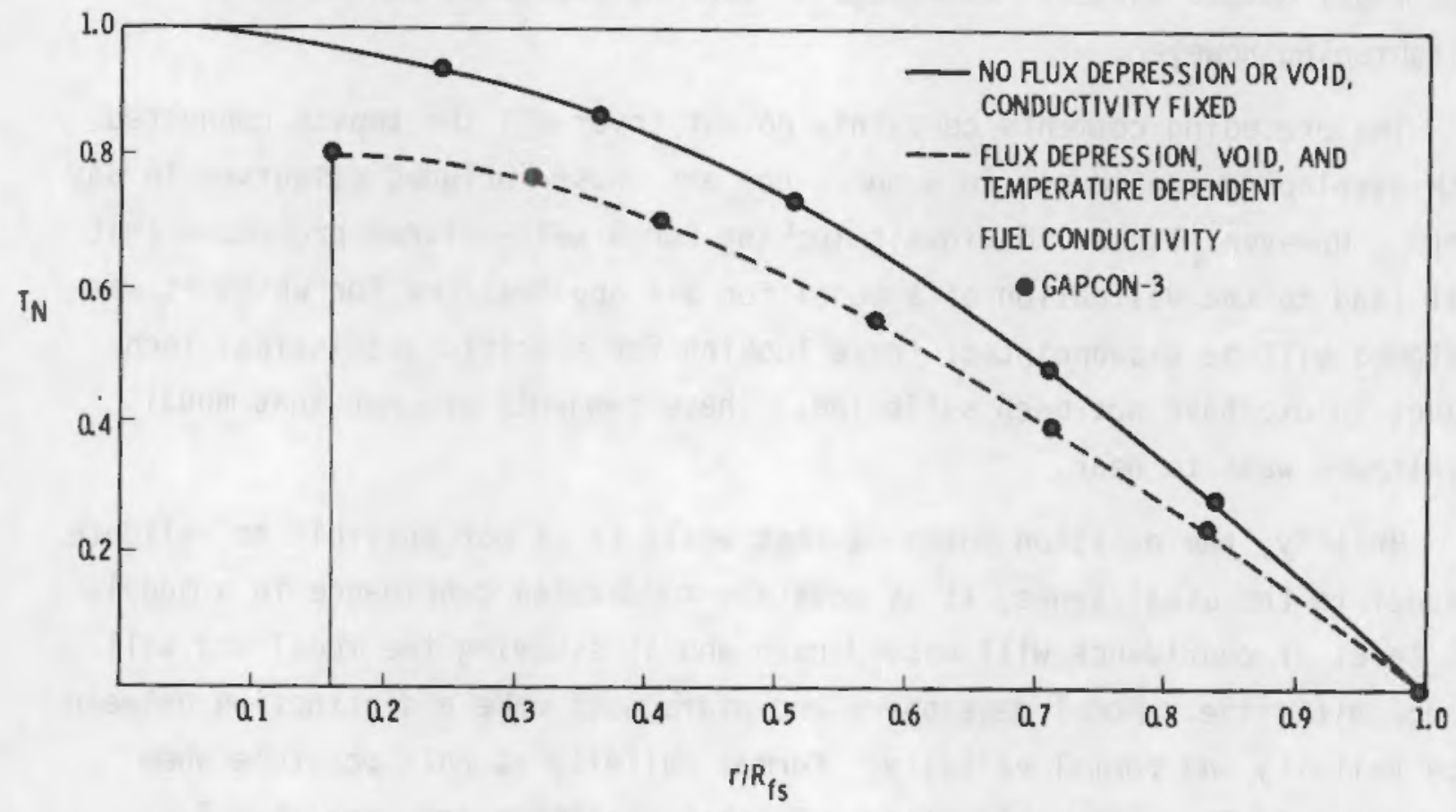

FIGURE 26. Verification of Steady-State Fuel Radial Temperature Profile Model

- Models used to describe separate effects, such as the thermal conductivity of fill gas, were validated by comparison to data. Figure 27 illustrates this type of validation.

- Statements on the validity of the entire code were prepared based on comparison to irradiation experiments. A series of defined tests were used in this comparison; Figure 28 provides an example of one comparison.

For each code/data comparison presented, a discussion of code usage and the experimental data were supplied to clarify and identify differences and similarities. Based on their work, the authors made the following general conclusions:

- mathematical consistency - The temperature calculations were accurate relative to analytical solutions; the mechanical models were consistent with a detailed finite element model. 


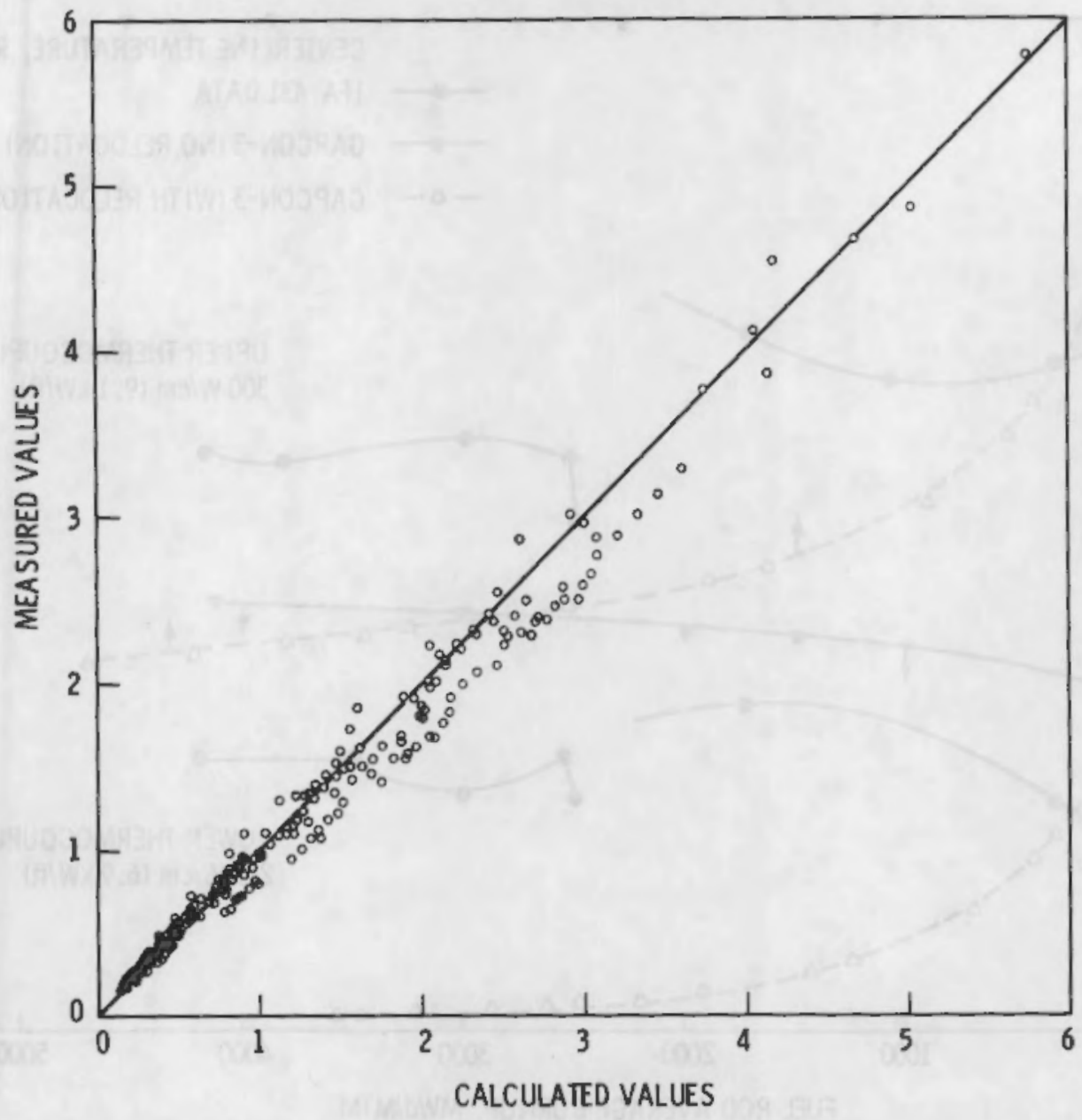

FIGURE 27. Validation of Gas Thermal Conductivity Model

- individual model validation - Comparison of the major models with recognized data sets showed that the models were contributing reasonable results to the overall calculation.

- total model validation - General agreement between code and data was obtained with some exceptions (which were listed).

This validation exercise provided no concrete limits on the usage of the code, and no statistical analysis was performed on the differences between code and data. 


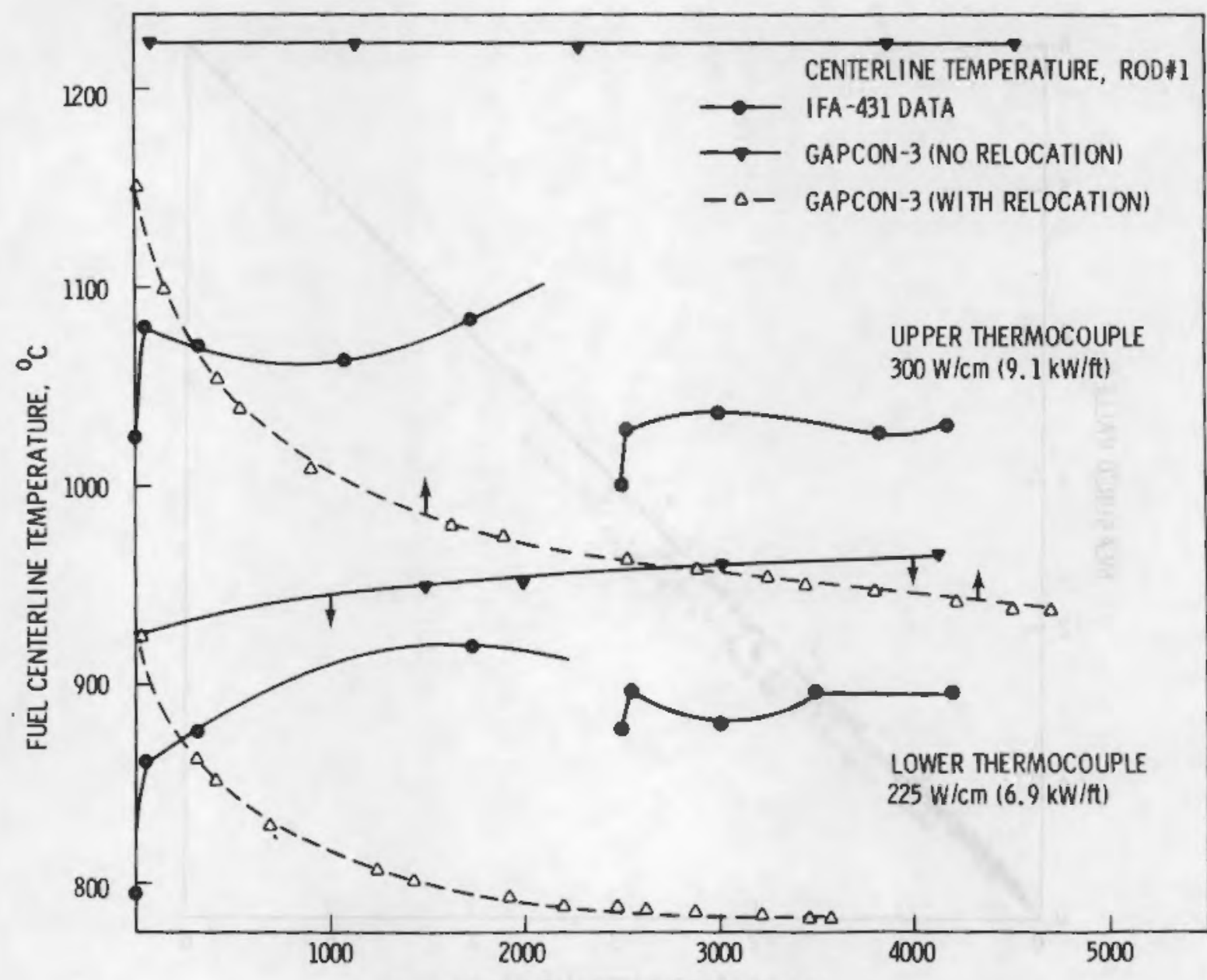

FUEL ROD AVERAGE BURNUP, MWd/MTM

FIGURE 28. Example of Validation of a Fuel Performance Code

The stated objectives for the assessment of FRAPCON-1 (Laats, Peeler, and Scofield 1979) were to evaluate the general code performance characteristics (model analysis) and perform a code/data comparison to evaluate model accuracy (model validation). Code verification is not presented in either the assessment document or the description document (Berna and Bohn 1980).

Model analysis of FRAPCON-1 consisted of running FRAPCON-1 and FRAP-S3 (Dearien 1978) on a selected problem, which resulted in the identification of changes in predictions due to model differences between the two codes. 
To perform the FRAPCON-1 model validation, a large set of irradiation data was compiled and FRAPCON-1 predictions were obtained. Two major forms of comparison are presented: plots of prediction versus data and plots of the difference between prediction and data. The resulting plots consist of bands that can be statistically analyzed to provide error estimates for the code relative to data.

Because of the large number of code/data comparisons, the FRAPCON-1 assessment used a substantial amount of computer time compared to the GT3 verification. In addition, it becomes more difficult to provide an estimate of the quality of each individual piece of data. However, it can also be said that this type of comparison provides a fair definition of the bounds and limitations of the code.

The third example is not truly a model validation exercise but an attempt to determine which of several fuel behavior codes was better. Freeburn, Pati, and Fiero (1977) compared six codes against five sets of irradiation data. The report presents a discussion of the codes, the data, the code predictions, and an evaluation of the performance of the codes.

The method of evaluation was to observe the differences between code predictions and experimental data. The following parameters were used:

- The bias (B):

$$
B=\frac{\sum W(P-E) / U}{N}
$$

where $P=$ the prediction

$E=$ the experimental value

$U=$ the absolute uncertainty of the experimental value

$W=$ the relative weight (importance) of the experimental value $\mathrm{N}=$ the number of comparisons.

- The standard deviation of the differences $\left(R^{*}\right)$ :

$$
R^{*}=\frac{\sum W\{[(P-E) / U]-B\}}{N}
$$


The final ranking of the codes was based on the values of $R^{\star}$ by assuming that a minimum value of $R^{\star}$ identified the best overall code.

This method of evaluation is useful in evaluating the integral response of a code and identifying any consistent bias in the integral behavior of the code relative to the data. However, the results are highly dependent upon the choice of weighting factors and uncertainties. It is quite possible that by changing weights and uncertainties and making a minor change to one or more codes the final ranking of the codes could change. In addition, the analysis only identified the code with the minimum difference to the data. To be used as a model validation tool, it would have been necessary to specify what the minimum acceptable values of $B$ and $R^{\star}$ would be.

The final validation example is different from the examples already reviewed. The three previous examples have compared code predictions to data from single rods irradiated under different conditions. There are now data available from a test assembly that had three identical rods. Thus, rather than comparing a code to data and estimated uncertainty, it is possible to compare a code to the observed variability between supposedly identical rods. (a) An observed band of data from nominally identical conditions should provide a better basis for code validation than a band of data from known differing conditions.

In this type of comparison, the uncertainty band for the code prediction should be interpreted as follows: Given the assigned uncertainty for code input and models, experimental data would be expected to fall within the code uncertainty band if the fuel rod was behaving according to the models in the code. If the code and data bands overlap, then it may be said that the difference between code and data has a low level of significance; however, if the data and code bands do not overlap, there is a high significance to the code/ data difference. It then becomes a problem of deciding if the code had the wrong input, if the data were observed incorrectly, or if the wrong models were used in the code.

(a) Obviously the rods are not truly identical. Manufacturing tolerances and localized differences in neutron flux will comoine to produce slightly different response between fuel rods. 
The third fuel assembly to be irradiated under the Experimental Support and Development of Single-Rod Fuel Codes Program is IFA-513 (Bradley et al. 1979). It contains three nominally identical rods of BWR design, and each rod contains two centerline thermocouples for data acquisition. Figure 29 compares the observed data from IFA-513 during its startup (Cunningham and Lanning 1978) with the predicted behavior from GT3. The variance bands on the data are calculated as

$$
\bar{x}=\frac{\sum x_{i}}{N}
$$

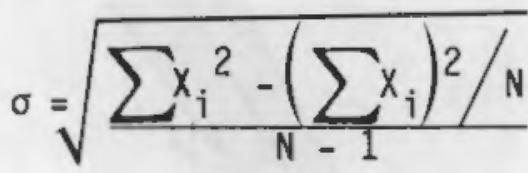

where the $x_{i}$ are the individual observations and $N$ is the total number of observations. The uncertainty band for the code prediction is based on STORE, using input uncertainties appropriate to the Halden Boiling Water Reactor (PNL-2581).

A second code has also been run against the IFA-513 data. This code, FRAPCON-1, is the first of a new series of audit codes currently being developed and used by the Fuel Behavior Research Branch of the NRC. Figure 30 compares this code to the data. Because FRAPCON-1 attempts to account for cracked fuel effects, the following changes to STORE were necessary:

- A fuel thermar conductivity multiplier equal to that used in FRAPCON-1 was used.

- Fuel thermal conductivity uncertainty was increased to $25 \%$ to account for the uncertainty of the multiplier.

Several observations can be made from Figures 29 and 30 . First, both GT3 and FRAPCON-1 overpredict the measured temperatures for IFA-513, with FRAPCON-1 predicting the higher temperatures. Second, when assuming a $10 \%$ uncertainty for fuel thermal conductivity, there is a significant difference between code predictions and observed behavior (the predicted uncertainty bands for the codes and the observed variance band for the data do not 


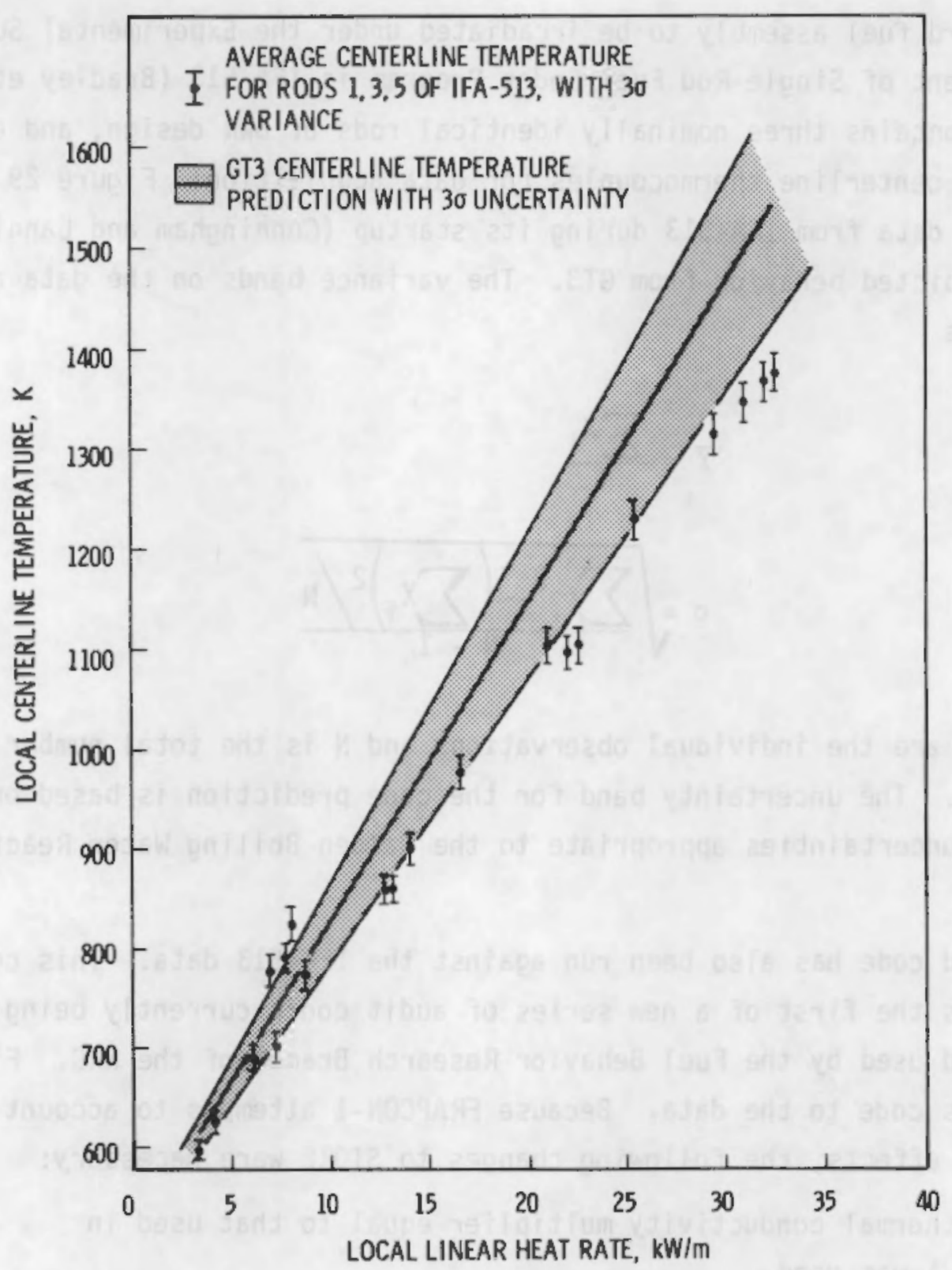

FIGURE 29. Comparison of GAPCON-THERMAL-3 to IFA-513 Startup Data 


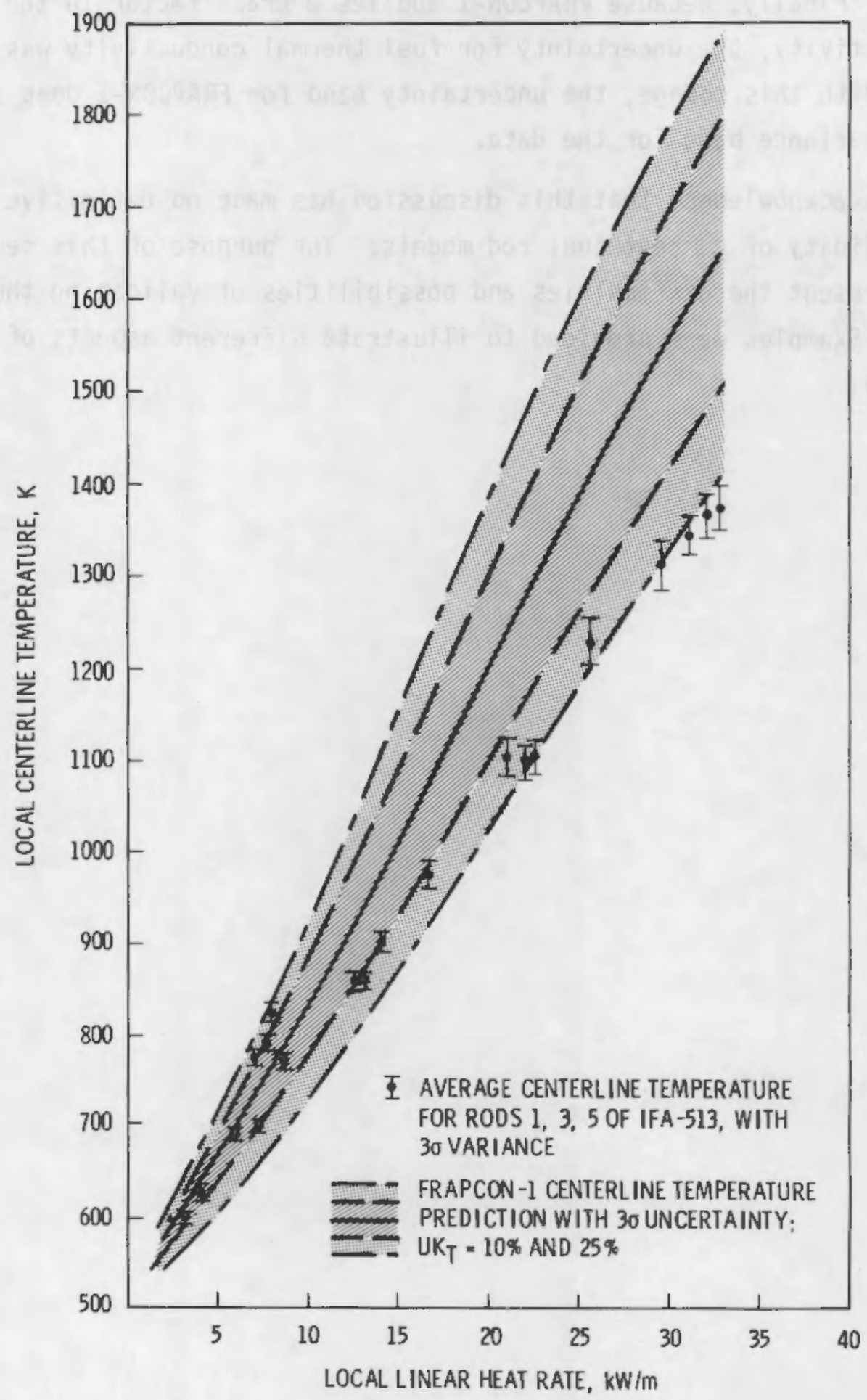

FIGURE 30. Comparison of FRAPCON-1 to IFA-513 Startup Data 
overlap). Finally, because FRAPCON-1 applies a crack factor to the fuel thermal conductivity, the uncertainty for fuel thermal conductivity was increased to $25 \%$. With this change, the uncertainty band for FRAPCON-1 does overlap the observed variance band for the data.

It is acknowledged that this discussion has made no definitive statements on the validity of current fuel rod models. The purpose of this section has been to present the difficulties and possibilities of validating the modeling process. Exampies were provided to illustrate different aspects of the discussion. 


\section{CRACKED PELLETS AND UNCERTAINTY ANALYSIS}

Until recently fuel modelers have made the assumption that fuel behaves as a solid right-circular cylinder concentrically located within the cladding. Although modelers have recognized that the fuel cracks, the assumption of concentric cylinders has been made for simplicity; and it has generally been adequate for thermal calculations but not always for the corresponding mechanical predictions. Because of this increasing awareness that the concentric cylinder model is limited, modeling efforts are now turning toward the problem of improving the interrelation of thermal and mechanical models. This section examines one such new model and its effect upon predicted uncertainty for temperatures and stored energy.

Macrographs of irradiated fuel have consistently shown a network of radial and circumferential cracks in the fuel. However, until recently only limited effort has been made to account for the effects those cracks may have upon the thermal and mechanical response of fuel rods. To prepare for the development of cracked pellet models, the Single-Rod Fuel Codes Program has been collecting transient temperature data for use in evaluating fuel rod behavior (Cunningham, Lanning, and Montgonery 1979). It has been concluded from model/data comparisons that a simplified cracked pellet model provides a better match with the transient temperature data than does the solid pellet model.

A cracked pellet model has been developed that utilizes "effective" values of fuel thermal conductivity and elastic modulus. These effective values are a function of the state of the fuel rod (Williford et al. 1980). This model, to be used in FRAPCON-2, (a) has the following basic features:

- Themal stresses cause the fuel to crack and relocate outward, thereby resulting in a smaller fuel-cladding gap than predicted by thermal expansion of a solid pellet alone.

(a) FRAPCON-2 has not yet been released; September 1980. 
- Because of relocation, the fuel remains in constant contact with the cladding. The contact pressure is determined by equating the fuel hydrostatic stress to the radial stress at the gap.

- The effective fuel themal conductivity and elastic moduli (radial and axial) are dependent on the cracks that exist within the fuel. As power and temperature increase, decreasing crack width (due to thermal expansion) increases the interaction between pellet fragments and therefore increases the effective thermal conductivity and elastic moduli.

In essence, the thermal and mechanical calculations remain based on the solid pellet model (for ease of calculation), but gap conditions and fuel properties (thermal and mechanical) are controlled by the available volume, which is in turn controlled by temperature and crack geametry.

This new model can be seen to affect uncertainty predictions in the following ways:

- Water film and cladding temperatures, and their uncertainty, are unaffected.

- The minimum value of gap conductance is equivalent to a closed gap with no interfacial pressure. Therefore, the uncertainty for gap size and gap conductance is reduced.

- The fuel thermal conductivity $\left(K_{F}\right)$ is reduced by applying a multiplier $\left(C_{F}\right)$ to the conductivity integral. Since there is an uncertainty for the multiplier (in addition to the uncertainty for the conductivity integral), the fuel thermal conductivity uncertainty is increased. This in turn changes the uncertainty predictions for fuel temperatures.

- The calculational method for stored energy is unchanged; however, the temperature profile and uncertainties are changed. Therefore, the predicted stored energy and its uncertainty will change. 
Since by definition the thermal conductivity multiplier is the ratio of cracked pellet thermal conductivity to solid pellet thermal conductivity, it follows that the value of $C_{F}$ ranges from 0.0 to 1.0. For specific values of $T_{S}$ and $T_{C L}, C_{F}$ is found from

$$
C_{F}=\frac{\frac{g F}{4 \pi}}{\int_{T_{S}}^{T_{C L}} K_{F}(T) d T}
$$

Thus, if $T_{C L}$ is known (data or calculation) and a minimum $T_{s}$ is specified, the calculated value of $C_{F}$ is a minimum (i.e., maximum thermal conductivity reduction).

Figure 31 presents minimum $C_{F}$ values for BWR $8 \times 8$ and PWR $17 \times 17$ rod designs as a function of rod total thermal resistance. The principal assumptions needed to derive the curves in this figure are coolant temperature, temperature rise across the fuel-cladding gap, and power level. Table 13 lists the power, fuel surface temperature, and centerline temperature for $C_{F}=1.0$. It should be recalled that an increase in resistance for a constant power means an increase in centerline temperature.

The curves in Figure 31 define the lower boundary of the interaction region between $C_{F}$ and thermal resistance $\left(C_{F}=1.0\right.$ is the upper boundary). Since the difference in temperature between centerline and fuel surface is inversely proportional to fuel thermal conductivity (i.e., as $K_{F}$ increases, $\Delta T=T_{C L}-T_{S}$ decreases), changes in $C_{F}$ will dictate changes in fuel temperature. Thus, as $C_{F}$ increases from the minimum for a constant resistance (constant centerline temperature), the fuel surface temperature will increase. On the other nand, if $C_{F}$ increases and $T_{S}$ is fixed, $T_{C L}$ and thermal resistance will decrease.

The uncertainty for fuel thermal conductivity (UK $K_{F}$ ) is a combination of the uncertainty for the solid thermal conductivity and the uncertainty for the conductivity multiplier. The total uncertainty is found from the two contributions by: 

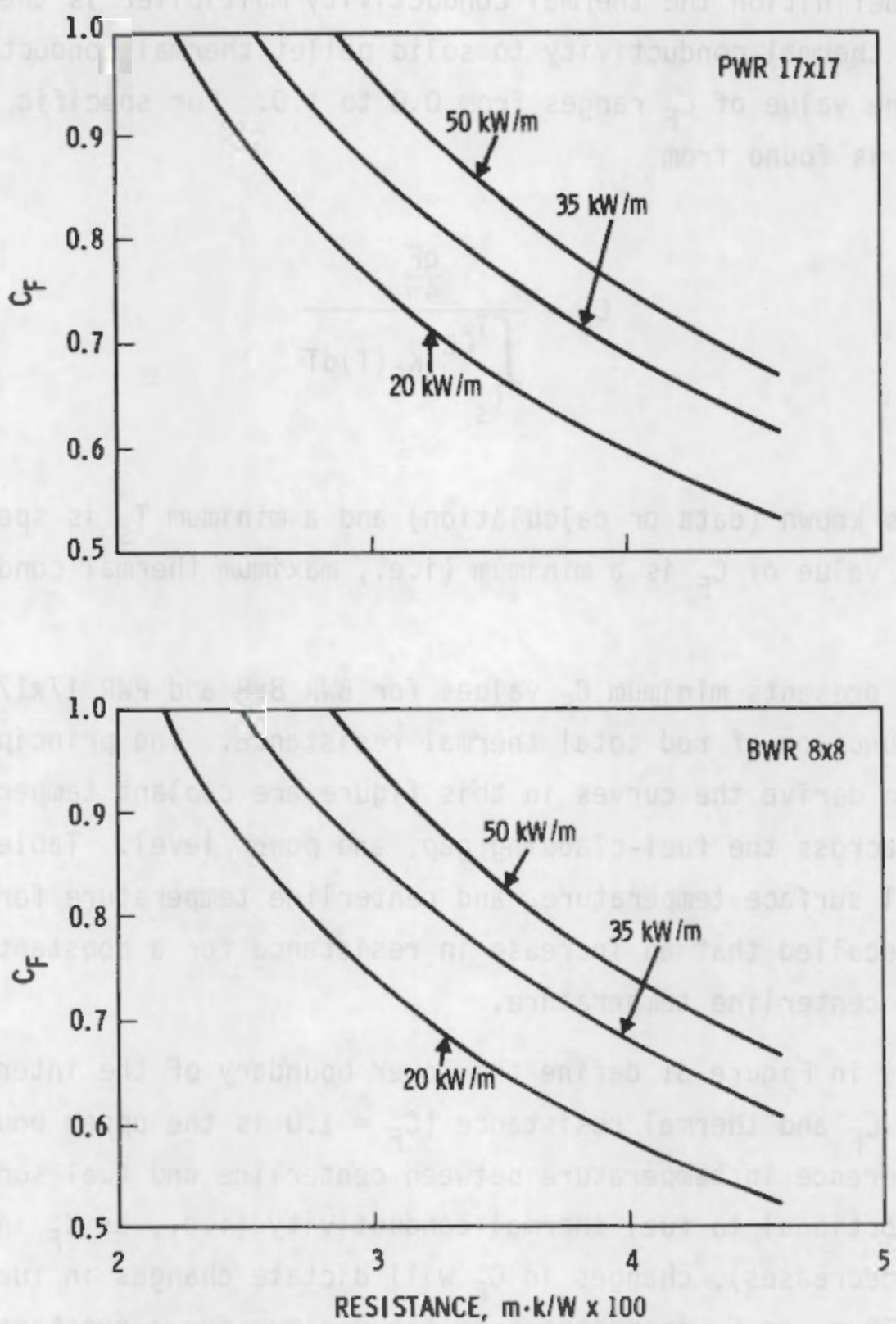

FIGURE 31. Minimum Conductivity Multiplier Values as a Function of Thermal Resistance 
TABLE 13. Assumptions of Parametric Plots of Minimum $\mathcal{C}_{F}$ Versus Resistance

\begin{tabular}{|c|c|c|c|}
\hline Rod Type & $\begin{array}{l}\text { Power, } \\
\mathrm{kW} / \mathrm{m}\end{array}$ & $\begin{array}{c}\text { Fuel Surface(a) } \\
\text { Temperature, } \\
\text { K }\end{array}$ & $\begin{array}{l}\text { Center line(b) } \\
\text { Temperature, } \\
\mathrm{K}\end{array}$ \\
\hline \multirow[t]{3}{*}{ BWR: $\quad T_{W}=553 \mathrm{~K}$} & 20 & 619 & 990 \\
\hline & 35 & 646 & 1424 \\
\hline & 50 & 673 & 1961 \\
\hline \multirow{3}{*}{ PWR: $\quad T_{w}=578 \mathrm{~K}$} & 20 & 15 37 & 1026 \\
\hline & 35 & 667 & 1470 \\
\hline & 50 & 693 & 2013 \\
\hline
\end{tabular}

(a) Fuel surface temperature calculated assuming $\Delta \mathrm{T}_{\mathrm{g}}=30 \mathrm{~K}$

(b) $\mathrm{C}_{\mathrm{F}}=1.0$; Lyons thermal conductivity

$$
U K_{F}=\left(U K_{F-S o l i d}{ }^{2}+U C_{F}{ }^{2}\right)^{1 / 2}
$$

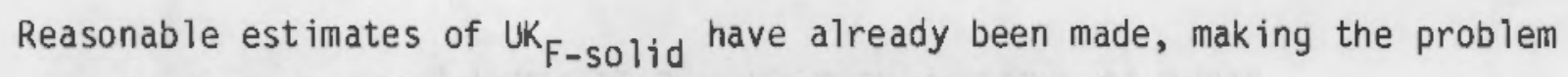
one of estimating the conductivity multiplier uncertainty.

In Figure 31 the upper and lower boundaries for the conductivity multiplier were developed as a function of total thermal resistance. By using these boundaries it is possible to define the maximum uncertainty for $C_{F}$. The relative uncertainty may be specified as the ratio of the difference between the maximum $\left(c_{F}=1.0\right)$ and minimum multiplier $\left(c_{F}=c_{F-m}\right)$ to the assumed value of the multiplier

$$
U C_{F}(x)=\frac{\left(1-C_{F-m}\right)}{C_{F}} \cdot 100
$$

Thus, for a rod with low thermal resistance and small void volume, $C_{F}$ is near 1.0 and has a small relative uncertainty. For a rod with high resistance, the range of possible $C_{F}$ values is larger and the relative uncertainty for the multiplier is larger. 
To show the effect upon uncertainty due to cracked pellet modeling, two rod designs were evaluated. Using the BWR $8 \times 8$ and PWR $17 \times 17$ power/temperature histories discussed in the Fuel Rod Design Analysis section, three STORE runs were made:

- solid pellet: $C_{F}=1.0$, varying $\Delta T_{g}, U_{F}=10 \%$

- cracked pellet: $C_{F}=$ minimum, $\Delta T_{g}=30 \mathrm{~K}, U_{\mathrm{F}}=10 \%$

- cracked pellet: $C_{F}=$ minimum, $\Delta T_{g}=30 K, U K_{F}=20 \%$.

For each run, the centerline temperature history matched that presented earlier.

The resulting centerline temperature and stored energy relative uncertainties are approximately constant with burnup; the range of values seen in Table 14 occurs throughout the burnup history. Notice that there is no overlap for the predicted relative uncertainties; and, as would be expected, the cracked pellet modeling has higher relative uncertainties predicted for centerline temperature and stored energy. In addition, relative uncertainties increase as the input fuel thermal conductivity uncertainty increases.

TABLE 14. Effect of Cracked Pellet Model on Relative Uncertainties

\% Uncertainty $(3 \sigma)$

BWR $8 \times 8 \quad$ PWR $17 \times 17$

Centeriine Temperature

$\begin{array}{lll}\text { A } & 14.9-16.0 & 15.0-16.0 \\ \text { B } & 16.6-17.7 & 15.6-16.8 \\ \text { C } & 18.0-20.0 & 17.0-18.3\end{array}$

Stored Energy

$\begin{array}{lll}\text { A } & 18.7-22.5 & 20.5-21.9 \\ \text { B } & 22.5-23.2 & 22.3-22.7 \\ \text { C } & 24.1-25.5 & 23.8-24.5\end{array}$

A: Solid Pellet $U_{K}=10 \%$

8: Cracked Pellet UKF $=10 \%$

C: Cracked Pellet UKF $=20 \%$ 
Although the relative uncertainty for stored energy is higher for the cracked pellet model than for the solid pellet model, the upper absolute bound ${ }^{(a)}$ for stored energy is lower for the cracked pellet model. The cracked pellet, with its lower fuel surface temperature, has a lower volumetric average fuel temperature and thus a lower stored energy. In general, the stored energy is sufficiently lower for the cracked pellet so that its higher value of relative uncertainty will not result in the absolute uncertainty bound exceeding that of the solid pellet. This effect is illustrated by plotting the predicted stored energy (Figure 32 ) and the three associated upper absolute bounds (Figure 33) for the BWR $8 \times 8$ power history.

Obviously, it would be possible for the cracked pellet upper absolute uncertainty bound to exceed the solid pellet upper absolute uncertainty bound if larger input uncertainties are used during cracked pellet uncertainty analysis. However, the values presented here are believed to be reasonable estimates of the uncertainties for cracked pellet modeling.

In summary, a cracked pellet model has the following effects on steadystate fuel rod temperature calculations relative to a solid pellet model:

- decreased fuel surface temperature because of the increased gap conductance

- decreased stored energy because of decreased fuel volumetric average temperature

- increased relative uncertainty for fuel centerline temperature and stored energy because of increased fuel thermal conductivity uncertainty

- decreased upper absolute uncertainty because of decreased stored energy.

(a) The absolute uncertainty bound is calculated from the nominal value and relative uncertainty by: $U_{X}$ (absolute) $=X_{\text {nominal }}$ * $\left[\left(1.0 \pm U_{X}\right.\right.$ (relative) $]$. 


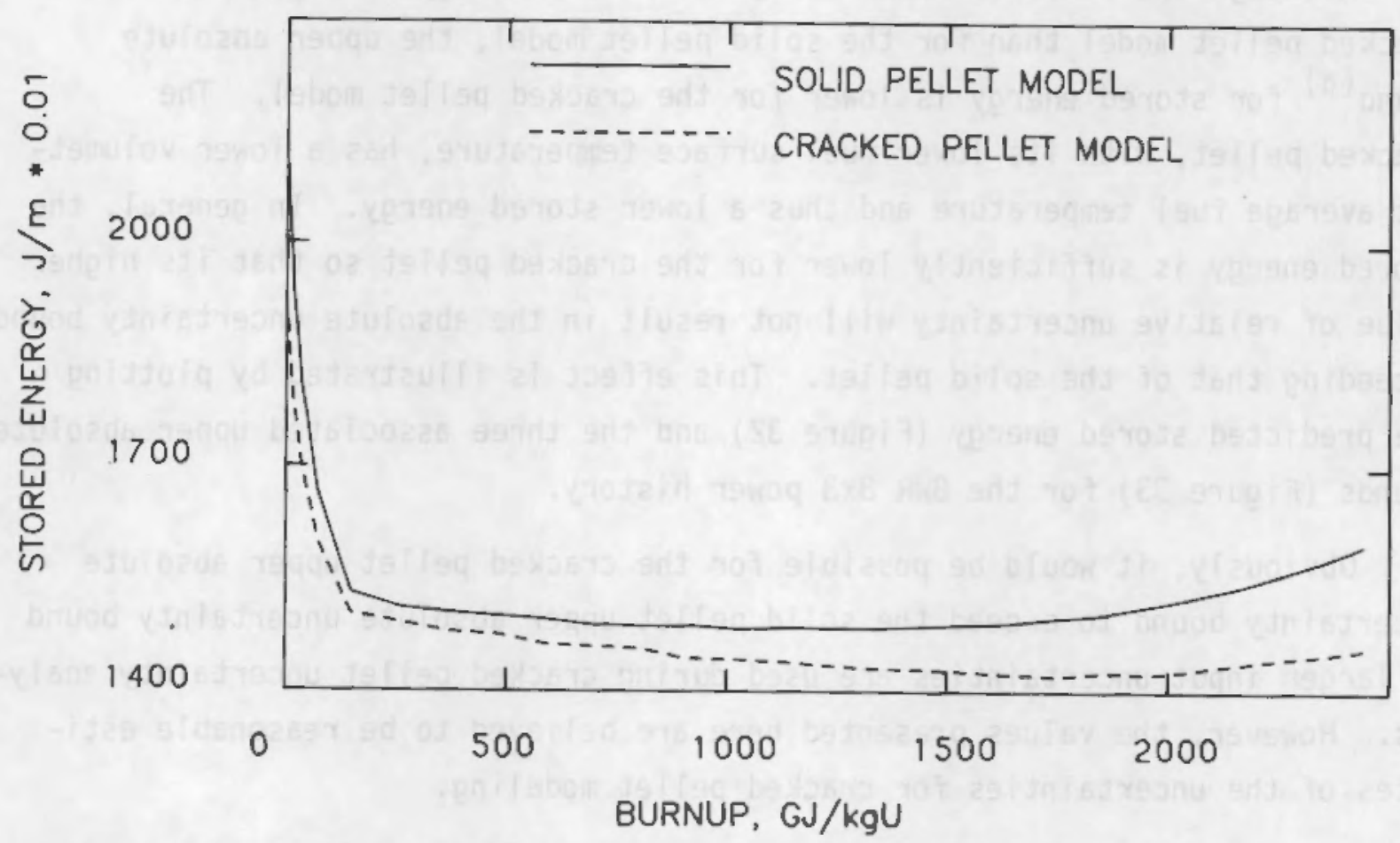

FIGURE 32. BWR $8 \times 8$ Stored Energy Based on Solid and Cracked Pellet Models
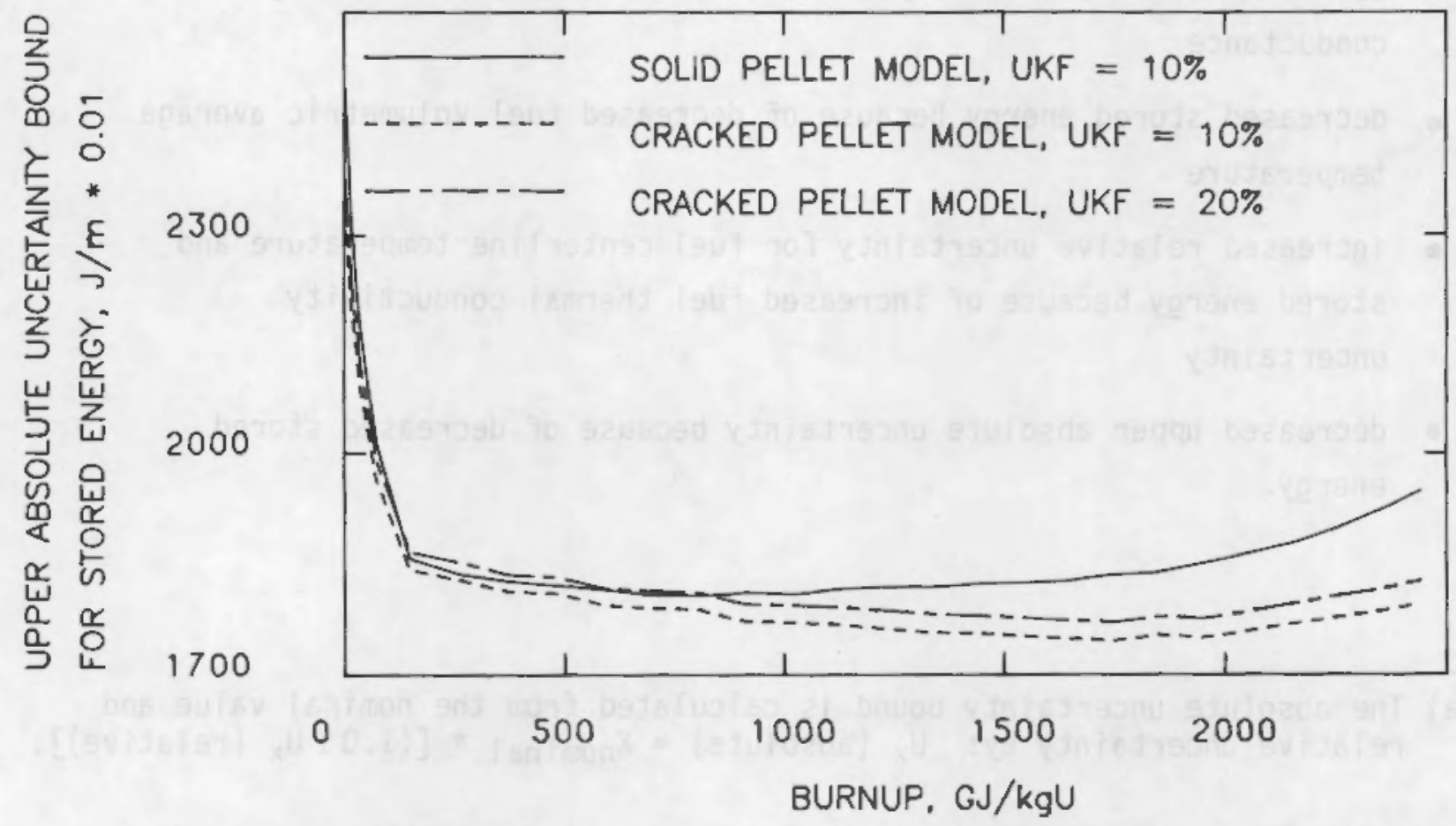

FIGURE 33. BWR $8 \times 8$ Stored Energy Upper Absolute Uncertainty Bounds 


\section{REFERENCES}

Bellany, R. G., and J. B. Rich. 1969. "Grain-Boundary Gas Release and Swelling on High Burnup Uranium Dioxide." J. Nucl. Mat. 33:64-78.

Beyer, C. E., and C. R. Hann. November 1974. Prediction of Fission Gas Release From $\mathrm{UO}_{2}$ Fuel. BNWL-1875, Pacific Northwest Laboratory, Richland, Washington.

* Bradley, E. R., et al. November 1979. Precharacterization Report for

Instrumented Nuclear Fuel Assembly IFA-513. NUREG/CR-1077, PNL-3156, Pacif ic Northwest Laboratory, Richland, Washington.

* Cunningham, M. E., and D. D. Lanning. July 1979. Startup Data Report for NRC/PNL Halden Assembly IFA-513. NUREG/CR-0862, PNL-2948, Pacif ic Northwest Laboratory, Richland, Washington.

* Cunningham, M. E., D. D. Lanning, and S. D. Montgomery. October 1979. A Procedure for the Qualitative Interpretation of Fuel Centerline Thermocouple Response to Step-Power Decreases. NUREG/CR-1012, PNL-3096, Pacific Northwest Laboratory, Richland, Washington.

* Cunningham, M. E., et al. May 1978. Stored Energy Calculation: The State of the Art. NUREG/CR-0320, PNL-2581, Pacific Northwest Laboratory, Richland, Washington.

Dearien, J. A., et al. March 1978. FRAP-S3: A Computer Code for Steady-State Analysis of Oxide Fuel Rods, Volume 1, Analytical Models and Input Manual. TFBP-TR-164, EG\&G Idaho, Inc., Idaho Falls, Idaho.

Freeburn, H. R., S. R. Pati, and I. B. Fiero. March 1977. Light Water Reactor Fuel Rod Modeling Code Evaluation. EPRI-NP-369, Electric Power Research Institute, Palo Alto, California.

* Hagrman, D. L., and G. A. Reymann. February 1979. MATPRO - Version 11; A Handbook of Materials Properties for Use in the Analysis of Light Water Reactor Fuel Rod Behavior. NUREG/CR-0497, TREE-1280, EG\&G Idaho, Inc., Idaho Falls, Idaho.

* Hann, C. R., et al. November 1977. Test Design, Precharacterization, and Fuel Assembly Fabrication for Instrumented Fuel Assemblies IFA-431 and IFA-432. NUREG/CR-0332, BNWL-1988, Pacif ic Northwest Laboratory, Rich 1 and, Washington.

Kerrisk, J. F., and D. G. Clifton. 1972. "Smoothed Values of the Enthalpy. and Heat Capacity of $\mathrm{UO}_{2}$." Nuclear Tech. 16:531-535.

* Laats, E. T., G. B. Peeler, and N. R. Scofield. May 1980. Independent Assessment of the Steady State Fuel Rod Analysis Code FRAPCON-1. NUREG/CR-1339, Idaho National Engineering Laboratory, Idaho Fa7ls, Idaho. 
Lanning, D. D., et al. January 1978. GAPCON-THERMAL-3 Code Description. PNL-2434, Pacif ic Northwest Laboratory, Richland, Washington.

Lokken, R. 0., and E. L. Courtright. 1977. A Review of the Effects of Burnup on the Therimal Conductivity on $\mathrm{U0}_{2}$. BNWL-2270, Pac if ic Northwest Laboratory, Richland, Wasinington.

Lyons, M. F., et al. 1964. U0, Pellet Thermal Conductivity from Irradiation with Central Melting. GEAP-4624, General Electric Co., San Jose, Californía.

Mar, B. W. 1974. "Prooblems Encountered in Multidisciplinary Resources and Environmental Simulation Models Development." J. of Environmental Management 2:83-100.

McKay, M. D., R. J. Beckman, and W. J. Conover. May 1979. "A Comparison of Three Methods for Selecting Values of Input Variables in the Analys is of Output from a Computer Code." Technometrics 21:239-245.

Mitchell, T. J., and D. G. Wilson. November 1979. Energy Model Validation: Initial Perceptions of the Process. ORNL/CSD-50, Oak Ridge National Laboratory, Oak Ridge, Tennessee.

"Williford, R. E., et al. April 1980. Interim Report; The Analysis of Fuel Relocation for the NRC/PNL Halden Assemblies IFA-431, IFA-432, and IFA-513. NUREG/CR-0588, PNL-2709, Pac if ic Northwest Laboratory, Richland, washington.

*Available for purchase from the NRC/GPO Sales Program, U.S. Nuclear Regulatory Commission, Washington, D.C. 20555, and the National Technical Information Service, Springfield, Va. 22161 
APPENDIX A

THERMAL MODELS IN STORE 


\section{THERMAL MODELS IN STORE}

The fuel models that are used in STORE are discussed in this appendix. Any change in modeling from PNL-2581 will be noted; however, no changes have been made that require changes to the uncertainty equations listed in Appendix 8 of PNL-2581.

It should be kept in mind that STORE is not a history-dependent code; therefore, history-dependent parameters such as fill gas composition must be input to STORE from the code of choice. This simplifies the workings of STORE since elaborate models are not used; only the end result and uncertainty of those models are used.

The models used in STORE are

- Water Film Temperature Rise

The temperature rise across the water film $\left(\Delta T_{f}\right)$ is determined by

$$
\Delta T_{f}=\frac{q}{\pi d_{c o} h_{f}}
$$

where the water film heat transfer coefficient $\left(h_{f}\right)$ and cladding outer diameter $\left(d_{c o}\right)$ are obtained from the results of each time step of the code of choice and $q$ denotes the local linear heat rate.

- Cladding Temperature Rise

The temperature rise across the cladding $\left(\Delta T_{c}\right)$ is found by

$$
\Delta T_{c}=\frac{q \ln \left(d_{c 0} / d_{c i}\right)}{2 \pi K_{c}}
$$

where the cladding thermal conductivity $\left(\mathrm{K}_{\mathrm{c}}\right)$ is obtained from the code of choice and $d_{c i}$ is the cladding inner diameter. 
- Cladding Inner Surface Temperature

The temperature on the cladding inner surface $\left(T_{c}\right)$ is determined by summing the coolant temperature $\left(T_{W}\right)$, water film temperature rise, and cladding temperature rise

$$
T_{c}=T_{w}+\Delta T_{f}+\Delta T_{c}
$$

- Temperature Rise Across the Gap and Fuel Surface Temperature

The temperature rise across the gap between the fuel and cladding $\left(\Delta T_{g}\right)$ is determined by

$$
\Delta T_{g}=\frac{q}{\pi d_{g} h_{g}}
$$

where $d_{g}$ is the diameter of the gap and $h_{g}$ is the gap conductance. The fuel surface temperature $\left(T_{s}\right)$ is found from

$$
T_{s}=T_{c}+\Delta T_{g}
$$

Because the fuel surface temperature is determined from the temperature rise across the gap but is also required in the solution for the gap temperature rise, an iterative solution is required.

The gap conductance is defined as

$$
h_{g}=\frac{K_{g}\left(T_{g}\right)}{x_{e}}+h_{s}
$$

where $K_{g}\left(T_{g}\right)$ is the thermal conductivity of the gas in the gap evaluated at the average gap temperature $\left(T_{g}\right), x_{e}$ is the effective gap width, and $h_{s}$ is the conductance due to fuel-cladding contact. Obviously, $x_{e}$ and $h_{s}$ are dependent upon whether or not fuel-cladding contact has occurred. 
For no fuel-cladding contact, $x_{e}$ is the sum of the physical gap $\left(x_{p}\right)$ and the temperature jump distance (TJ). The physical gap is defined as

$$
X_{p}=\frac{d_{f i}}{2}\left(\sqrt{F_{p r}}-\sqrt{F_{p}}\right)+R
$$

where $d_{f i}$ is the initial fuel diameter, $F_{p}$ and $F_{p r}$ determine the reversible and permanent changes in fuel cross-sectional area, respectively, (a) and $R$ is the combined roughness of the fuel and cladding. The temperature jump distance is defined as

$$
T J=a_{g} K_{g}\left(T_{g}\right) \sqrt{T_{g}+273}
$$

where $a_{g}$ is a coefficient. The solid conductance $\left(h_{s}\right)$ is zero.

For fuel-cladding contact, the effective gap width is defined as

$$
x_{e}=(R+T J) \exp \left(-C_{1} \Delta F_{p}\right)
$$

The solid conductance is found from

$$
h_{s}=c_{2} \Delta F_{p}
$$

where $\Delta F_{p}$ is the difference between the square roots of $F_{p}$ and $F_{p r}$. The coefficients $C_{1}$ and $C_{2}$ are determined by assuming limiting conditions for the minimum effective gap and the maximum solid conductance.

In PNL-2581, the gas thermal conductivity was determined from a cubic function that was input for each different gas mixture. To simplify the

(a) See PNL-2581 for a discussion of the derivation and meaning of $F_{p}$ and $F_{p r}$.
(b) Minimum gap width $=0.5 \mathrm{R}$ Maximum contact conductance $=0.0015 \mathrm{~W} / \mathrm{m}^{2}-\mathrm{K}$ 
use of STORE, the KGAS subroutine from GT3 (used to calculate gas thermal conductivity) has been added to STORE. It is now necessary to input only the appropriate gas mixture, rather than determining a conductivity function.

\section{- Fuel Centerline Temperature}

With the fuel surface temperature specified, it is possible to solve for the fuel temperature at any radius through the use of the integral conductivity method. Specifically, for the fuel centerline temperature

$$
\int_{T}^{T} C L K_{F}(T) d T=\frac{q F}{4 \pi}
$$

where the fuel thermal conductivity $\left(K_{F}\right)$ and flux depression $(F)$ are the same as used in the code of choice. (a)

- Stored Energy

The stored energy of the fuel $\left(E_{s}\right)$ relative to the coolant temperature may be simply stated as

$$
E_{s}=C_{p}\left(T_{a}\right)\left[T_{a}-T_{w}\right]^{\rho \pi d_{f f}} \frac{{ }^{2}}{4}
$$

where $C_{p}$ is the fuel specific heat, $T_{a}$ is the fuel volumetric average temperature, $\rho$ is the fuel density, and $d_{f f}$ is the hot fuel diameter. This does not account for any stored energy in the cladding or fuelcladding gap.

(a) GT3 and STORE use the thermal conductivity expression for $95 \%$ TD $\mathrm{UO}_{2}$ developed by Lyons (1964) and modified by the Maxwell-Eucken correction for densities other than 95\% TD. 
APPENDIX B

THERMAL RESISTANCE 


\section{APPENDIX B}

\section{THERMAL RESISTANCE}

Data from in-reactor fuel rod tests commonly consist only of power, coolant temperature, and fuel centerline temperature. As a result, only one thermal property can be determined from these parameter measurements: thermal resistance. By analogy with Ohm's Law, thermal resistance is the ratio of potential to current. More specifically, the total thermal resistance for a fuel rod is defined as the difference between the fuel centerline and coolant temperatures divided by the local linear power

$$
R_{T}=\frac{T_{C L}-T_{W}}{q}
$$

In a fuel rod, the total thermal resistance is the sum of resistances for the individual regions: cladding, gap, and fuel. Figure B.1 illustrates the general resistance versus power behavior for a typical boiling water reactor (BWR) fuel rod at beginning of life (BOL). The decrease in gap resistance is due to fuel thermal expansion closing the gap and increasing the gap conductance. The fuel resistance increases with power because fuel thermal conductivity decreases with temperature.

A fuel rod is classified as balanced if resistance is constant with power; fuel-dominated if resistance increases with power; or gap-dominated if resistance decreases with power (Figure B.2). As examples, a helium-filled rod with a small fuel-cladding gap will be identified as fuel dominated, while a xenon-

filled rod will be identified as gap dominated. Resistance versus power plots are therefore useful tools in inferring fuel rod behavior. 


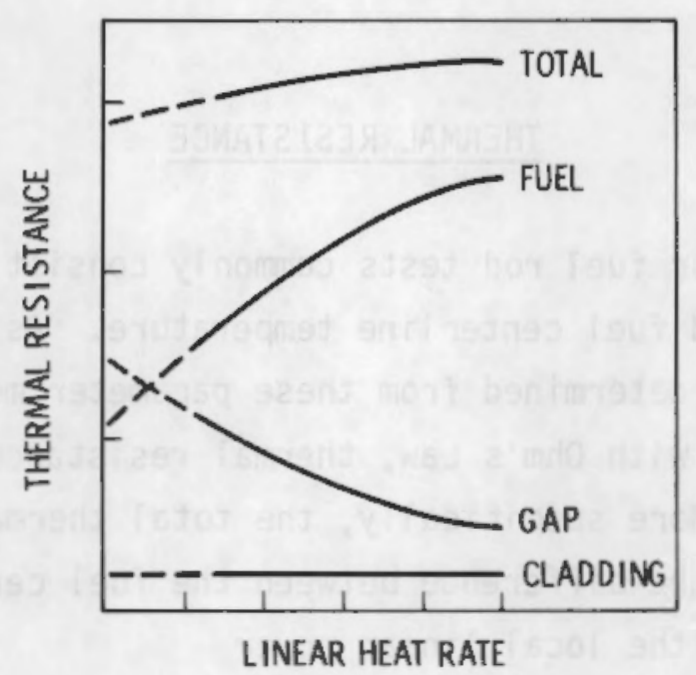

FIGURE B.1. Components of Resistance

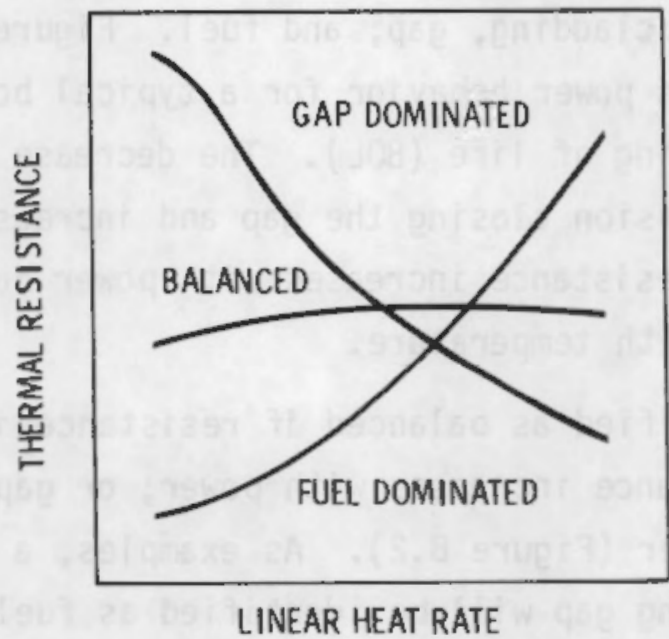

FIGURE B.2. Resistance Behavior of Various Rods 
Since $T_{C L}, T_{W}$, and $q$ are all separately measured during experiments, there are no covariances between these parameters; and the relative variance for resistance calculated from data may be found from

$$
\sigma_{R_{T}}^{2}=\sum_{j=1}^{P}\left(\frac{\partial R_{T}}{\partial X_{i}}\right)^{2} \sigma_{i}^{2}
$$

where the $x_{j}$ are the variables $T_{C L}, T_{W}$, and $q$, and the $\sigma_{i}^{2}$ are their variances. Relative variance may be defined as

$$
c_{i}^{2}=\frac{\sigma_{i}^{2}}{\mu_{i}^{2}}
$$

where $\mu_{i}$ is the mean value of $x_{j}$. The relative variance of the thermal resistance may be written as

$$
C_{R_{T}}^{2}=\frac{T_{C L}^{2}}{\left(T_{C L}-T_{W}\right)^{2}} C_{T_{C L}}^{2}+\frac{T_{W}^{2}}{\left(T_{C L}-T_{W}\right)^{2}} C_{T_{W}}^{2}+C_{q}^{2}
$$

The percent uncertainty $(3 \sigma=99.7 \%$ confidence leve 1$)$ for experimentally measured thermal resistance is then

$$
U_{R_{T}}=\sqrt[300]{C_{R_{T}}^{2}}
$$




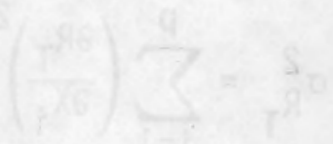




\section{APPENDIX C}

BURNUP-DEPENDENT FILL GAS COMPOSITION 
APPENDIX C

\section{BURNUP-DEPENDENT FILL GAS COMPOSITION}

This appendix provides a brief presentation of the change in fuel rod fill gas composition during burnup. Figures C.1 to C.5 present the change in gas composition that was predicted by GT3 for the Case 2 power histories discussed in the Fuel Rod Design Analysis section. The solid line in each figure is the predicted composition, and the dotted lines are the upper and lower composition boundaries based on the $1 \%$ est imate for gas release uncertainty. Figure C.6 illustrates the change in gas thermal conductivity that occurs as an increasing amount of fission gas (xenon and krypton) is mixed with the original helium. Note that as the percentage of fission gas increases, the rate of change of thermal conductivity decreases.

The results presented in Figures C.1 to C.5 were combined with Figure C.6 and used to determine gas thermal conductivity uncertainty for each burnup step (see Table 6 in text). It can be seen that the boiling water reactor (BWR) rods (Figures C.1 to C.3) have a possible wide variation in gas composition at a specific burnup and thus a large thermal conductivity uncertainty. At higher burnups the composition band begins to narrow, and the thermal conductivity curve is flatter; therefore, thermal conductivity uncertainty at endof-life (EOL) is lower. On the other hand, the larger initial amounts of helium in the pressurized water reactor (PWR) rods counteract fission gas release and result in a narrower gas composition band throughout the power history (Figures C.4 and C.5). Thus, the PWR rods have a lower gas thermal conductivity uncertainty. 


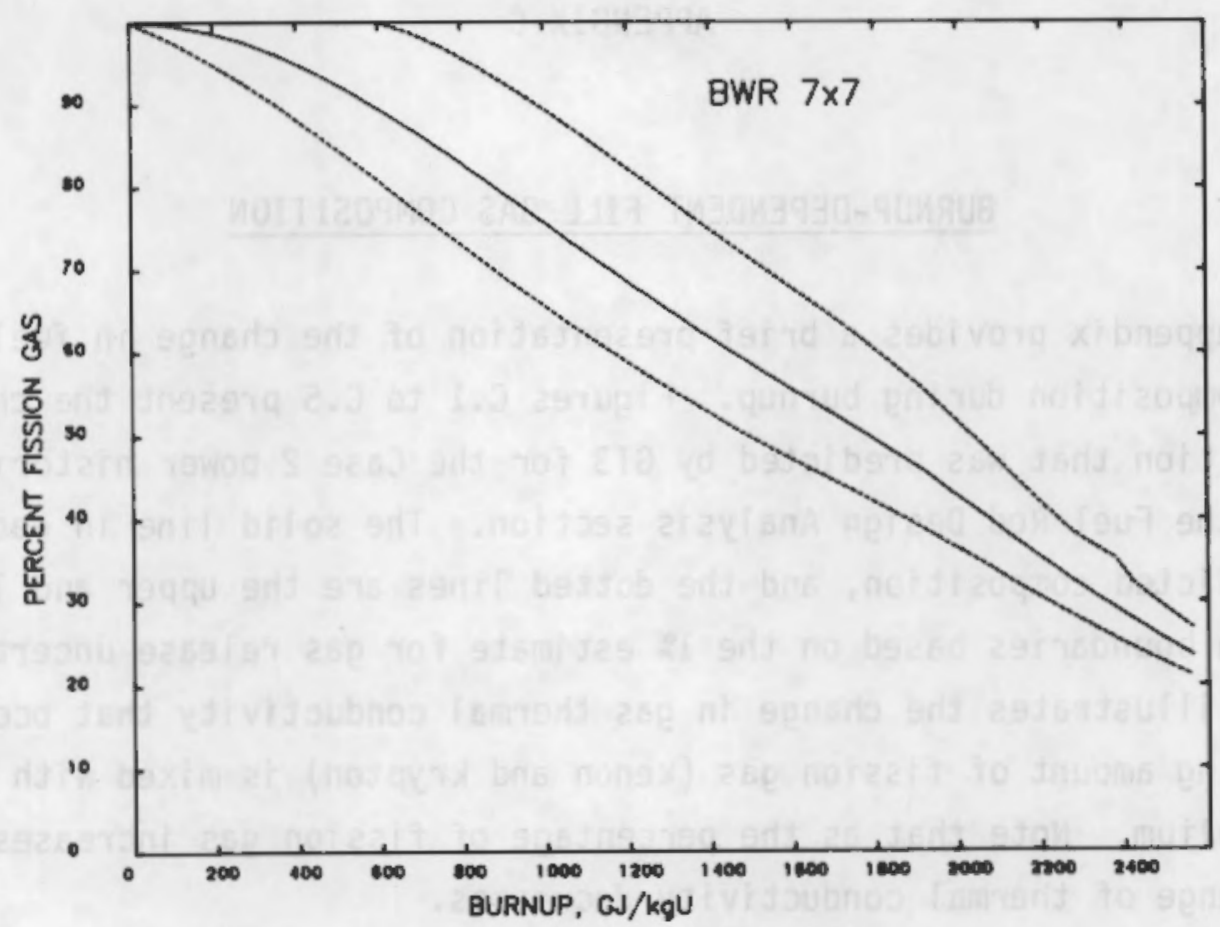

FIGURE C.1. Fill Gas Composition History for BWR $7 \times 7$, Case 2

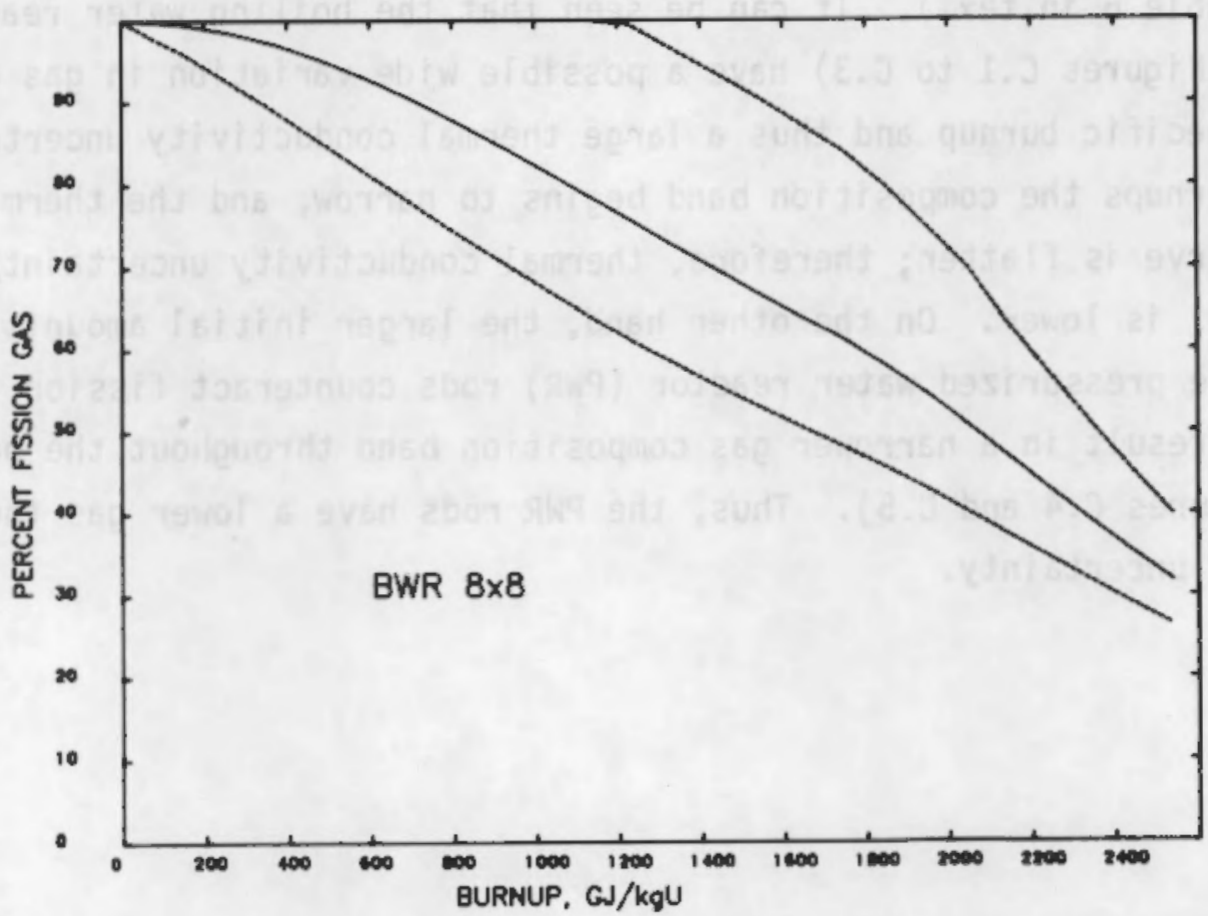

FIGURE C.2. Fill Gas Composition History for BWR $8 \times 8$, Case 2 


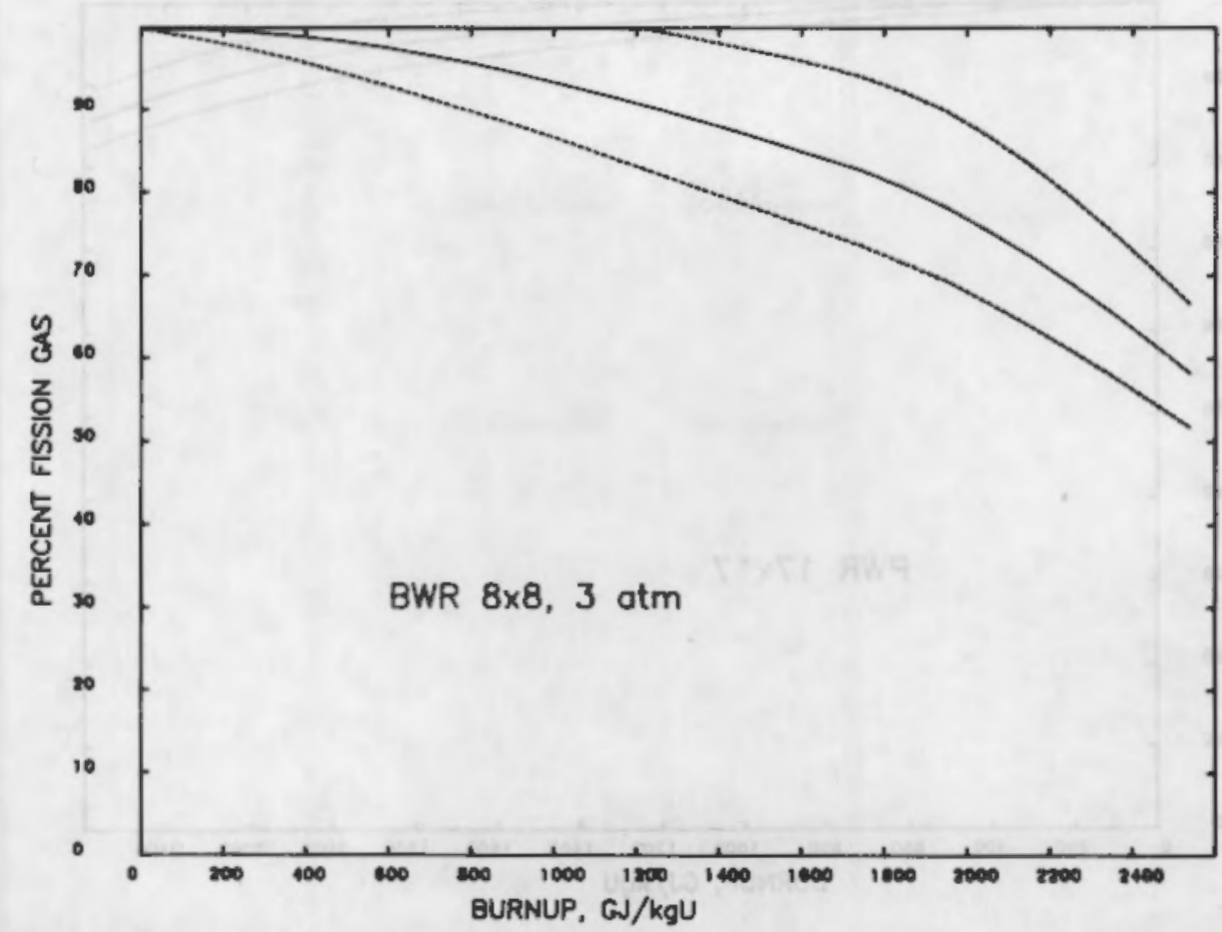

FIGURE C.3. Fill Gas Composition History for $3 \mathrm{~atm}$ BWR $8 \times 8$, Case 2

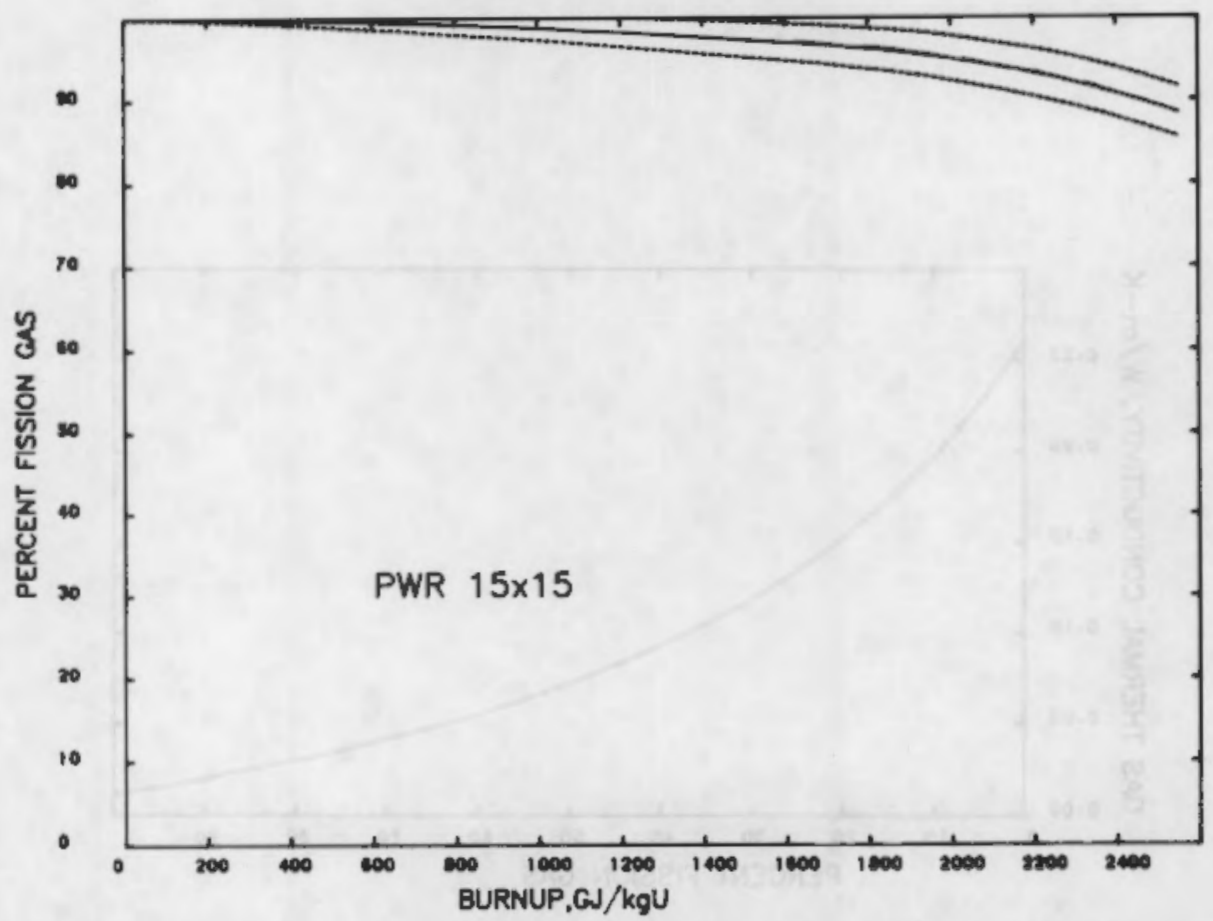

FIGURE C.4. Fill Gas Composition History for PWR $15 \times 15$, Case 2

$$
\text { C.3 }
$$




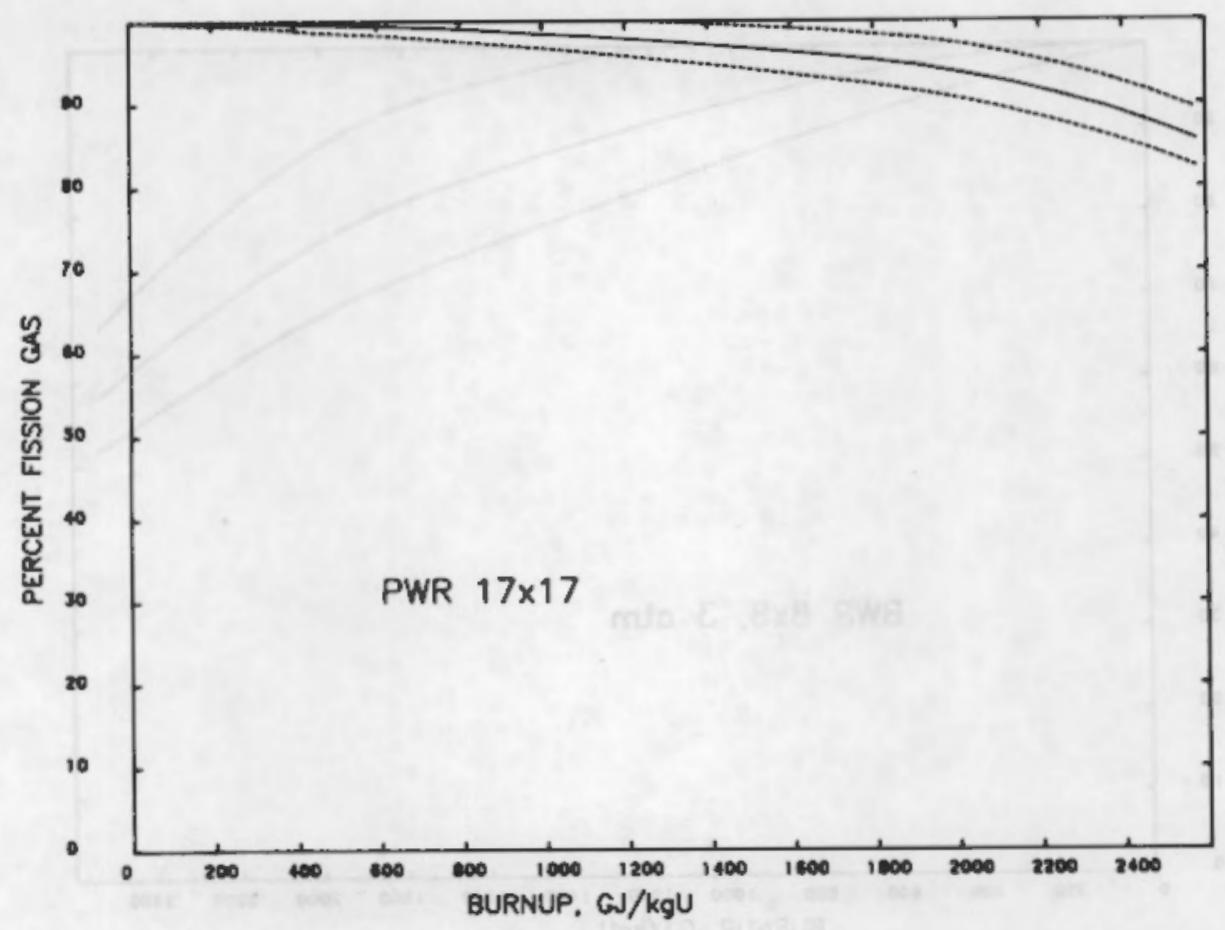

FIGURE C.5. Fill Gas Composition History for PWR 17x17, Case 2

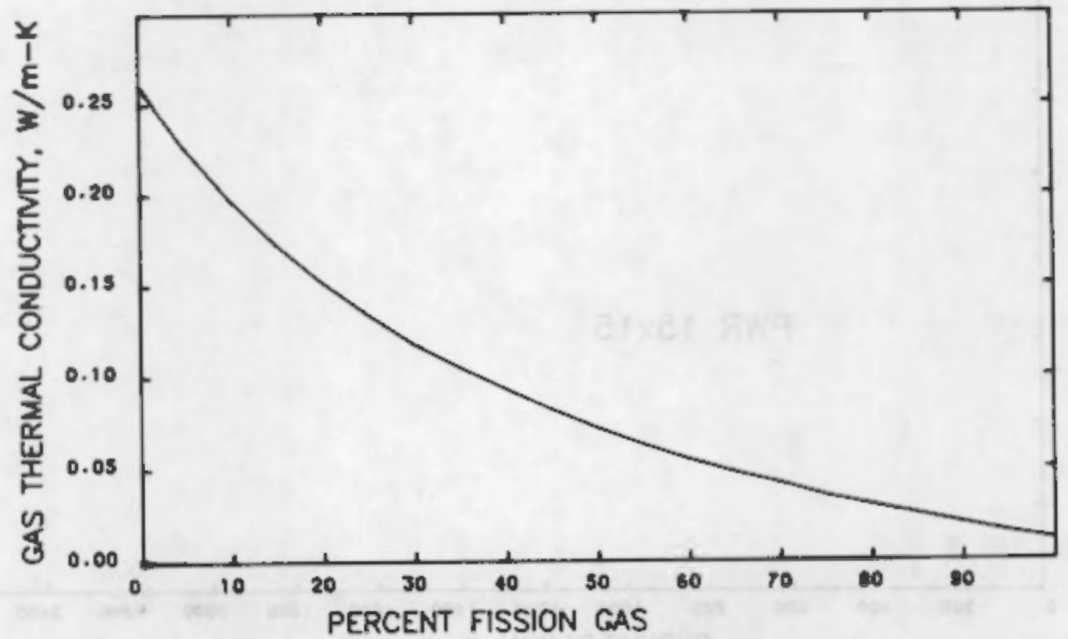

FIGURE C.6. Gas Thermal Conductivity as a Function of Fill Gas Composition at $673 \mathrm{~K}$ 


\section{DISTRIBUTION}

No. of

Copies

OFFSITE

A. A. Churm

DOE Patent Division

9300 S. Cass Avenue

Argonne, IL 60439

400 Nuclear Regulatory Commission

Division of Technical Inf ormation and Document Control

7920 Norfolk Avenue

Bethesda, MD 20014

2 DOE Technical Information Center

4 W. V. Johnston

Chief, Fuel Behavior Research Branch

Division of Reactor Safety

Research

U.S. Nuclear Regulatory Comnission Washington, D.C. 20555

H. H. Scott

Fuel Behavior Research Branch

Division of Reactor Safety Research

U.S. Nuclear Regulatory Commission Washington, 0.C. 20555
No. of

Copies

ONSITE

46 Pacific Northwest Laboratory

W. J. Bailey

J. 0. Barner

E. R. Bradley

M. E. Cunningham (20)

S. K. Edler

M. D. Freshley

R. L. Goodman

R. J. Guenther

C. R. Hann

K. A. Hsieh

D. D. Lanning

R. K. Marshall

C. L. Mohr

C. Nealley

A. R. 0lsen

F. E. Panisko

W. N. Rausch

R. E. Schreiber

R. E. Williford

Technical Inf ormation (5)

Publishing Coordination Fe(2) 


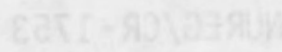

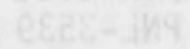

$\varepsilon=9$

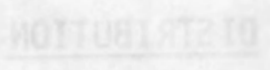

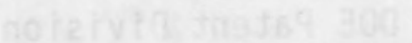

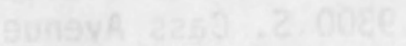

$\operatorname{ccta0}$

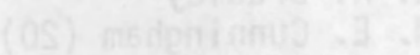

xefinagese

3450009

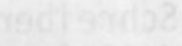

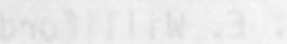

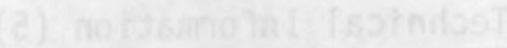

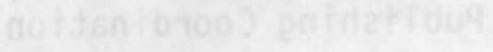

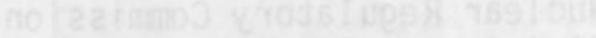

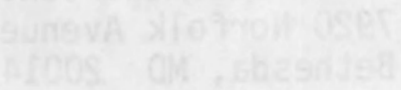

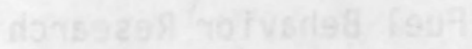

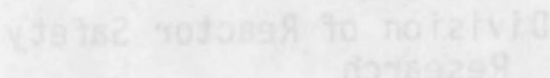

atcos

to

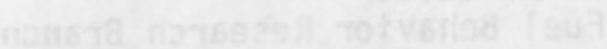

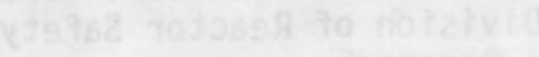

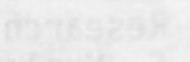

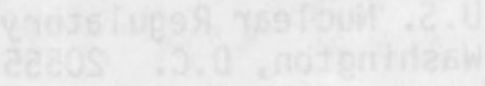


NRC FOAM 335

$(7.77)$

U.S. NUCLEAR REGULATORY COMMISSION

BIBLIOGRAPHIC DATA SHEET

1. REPORT NUMEER (Assigned by DOC)

NUREG/CR-1753

PNL -3539

4. TITLE AND SUBTITLE (A dd Volume No, if appropriare)

Application of Linear Propagation of Errors to Fuel Rod

Temperature and Stored Energy Calcualations

2. (Leave blank)

3. RECIPIENT'S ACCESSION NO.

7. A.E. Cunningham, A.R. 01sen, D.D. Lanning, R.E. Williford

9. PERFORMING ORGANIZATION NAME AND MAILING ADDRESS (Include Zip Code)

Pacific Northwest Laboratory

P.0. Box 999

Richland, Wa. 99352

5. DATE REPORT COMPLETED

\begin{tabular}{l|l}
\hline MONTH & YEAR
\end{tabular}

September $\quad 1980$

DATE REPORT ISSUED

MONTH

October

1980

6. (Leave blank)

8. (Leave biank)

12. SPONSORING ORGANIZATION NAME AND MAILING ADDRESS (Include Zip Cade)

Division of Reactor Safety Research

office of Nuclear Regulatory Research

10. PROJECT/TASK/WORK UNIT NO.

U.S. Nuclear Regulatory Commission

Washington, D.C. 20555

13. TYPE OF REPORT

PE AIOO COVE AEO (Inclusive dates)

15. SUPPLEMENTARY NOTES

14. (Leave olank)

16. ABSTRACT (200 words or less)

Linear propagation of errors evaluates modeling uncertainty by approximating a function of interest by first-order Taylor's series expansions and then approximating the variance of the function by the variance of the linear approximation. This report discusses uncertainty analysis for different nuclear fuel rod designs, the process of model validation, and the effect of cracked pellet fuel models upon temperature uncertainty.

Using a postulated power history, the uncertainty for the predicted thermal response of boiling water reactor (BWR) and pressurized water reactor (PWR) fuel rods was evaluated. Beginning-of-life (BOL) relative uncertainty for BWR and PWR fuel rods is approximately the same, while different end-of-life (EOL) thermal response results in different EOL uncertainty. Determining eh validity of modeling relative to reality is discussed in qualitative terms.

\begin{tabular}{|c|c|}
\hline $\begin{array}{l}\text { 19. SE CURITY CLASS (Thts report) } \\
\text { Unclassified }\end{array}$ & 21 NO OF PAGES \\
\hline 20. SECURITY CLASS (This page) & $\begin{array}{l}\text { 22. PRICE } \\
\mathrm{S}\end{array}$ \\
\hline
\end{tabular}


.

$\therefore-$ 